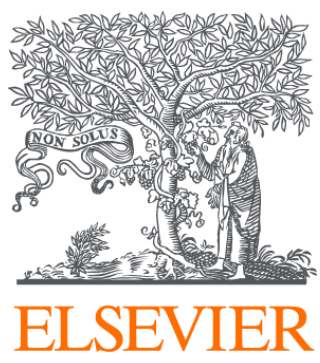

Since January 2020 Elsevier has created a COVID-19 resource centre with free information in English and Mandarin on the novel coronavirus COVID-

19. The COVID-19 resource centre is hosted on Elsevier Connect, the company's public news and information website.

Elsevier hereby grants permission to make all its COVID-19-related research that is available on the COVID-19 resource centre - including this research content - immediately available in PubMed Central and other publicly funded repositories, such as the WHO COVID database with rights for unrestricted research re-use and analyses in any form or by any means with acknowledgement of the original source. These permissions are granted for free by Elsevier for as long as the COVID-19 resource centre remains active. 


\section{Emergence of colistin-resistant Gram-negative Enterobacterales in the gut of patients receiving oral colistin and neomycin decontamination}

\section{To the Editor}

\section{Sir, Madam,}

In this Journal, Zhu et al. recently reported the results of their genomic analysis of multidrug-resistant Klebsiella pneumoniae isolates from individual patients before and after colistin treatment highlighting the rapid emergence and multifaceted molecular mechanisms of colistin resistance in $K$. pneumoniae. ${ }^{1}$ This work highlights the therapeutic and public-health challenges of colistinresistance (CR), which is increasingly used as a large resort antibiotic, despite its unattractive toxicity profile and narrow therapeutic window. ${ }^{2}$ Oral non-absorbed colistin has been proposed as a decontamination strategy in intensive care units and for patients carrying multidrug resistant Enterobacterales (MDR-E). ${ }^{3,4}$ The impact of decolonization strategies in terms of emergence of $C R$ has rarely been monitored because no reliable selective medium existed and $\mathrm{CR}$ was not considered a public-health problem. Recently, reliable universal culture media have been developed to screen for CR. ${ }^{5}$

Here, we studied the impact of non-absorbed oral colistin on the emergence of CR in the gut microbiota of patients from the RGNOSIS-WP3 randomized controlled trial. ${ }^{6}$ Thirty-nine subjects colonized with MDR-E were randomized to receive oral colistin sulfate 2 MIU 4 times a day + neomycin sulfate $500 \mathrm{mg}$ bid for 5 days followed by a fecal microbiota transplant (FMT) from healthy donors, or no intervention. Stool samples were collected on visit V0 (screening sample), V2 (after 5 days of oral decontamination and before FMT for the intervention group), V3, V4 and V5, respectively 5-10 days, 30-40 days and 150-210 days later. ${ }^{6}$ Stool samples from donors and 15 subjects from the intervention group and 15 from the control group were available for this work and plated on Drigalski plates (control) and SuperPolymyxin ${ }^{\circledR}$ plates. ${ }^{5}$ Colony forming units (CFU) counts of all Gram-negative rods were determined. Isolates growing on Superpolymyxin ${ }^{\circledR}$ plates were identified by MALDI-TOF; CR was confirmed by the culture-based Rapid Polymyxin NP test and MIC determined by the microdilution method. The limit of detection was $10^{2} \mathrm{CFU} / \mathrm{g}$ of stool. CR-E. coli were sequenced using the Illumina HiSeq technology. To determine whether CR isolates were present before the intervention, a specific mcr-1 PCR was performed on patients stool prior to intervention (V0) and on the donor's stool. ${ }^{7}$ Electroporation of plasmids was performed to localize the gene conferring resistance to colistin and neomycin and molecular typing of the electroporants was performed using PCR based replicon typing (PBRT).

\footnotetext{
Université de Paris, IAME, INSERM, UMR-1137
}

No patient or donor included in the trial carried CR isolates on V0. Among the 15 patients in the intervention group two (13.3\%, [IC90 $-1 ; 3$ ], $p=0.24$ ) carried CR isolates at least at one visit after the intervention (Fig. 1). No CR-Enterobacterales was detected in the stools of subjects from the control group. Among both subjects with CR- Enterobacterales, one carried $10 \mathrm{Log}$ CFU/g of Hafnia paralvei, a species which is intrinsically resistant to colistin $(\mathrm{MIC}=8 \mathrm{mg} / \mathrm{L})$, also resistant to neomycin $(\mathrm{MIC}=32 \mathrm{mg} / \mathrm{L})$ on visit 4 and the other carried $1 \mathrm{Log}$ CFU/g and 3 Log CFU/g CR-E. coli at visits 4 and 5 , respectively, with a colistin MIC at $8 \mathrm{mg} / \mathrm{L}$. Relative abundance of CR-E. coli increased between V4 and V5 from $0.01 \%$ to $1 \%$ of the total Enterobacterales population. The CR-E. coli recovered at V4 and V5 both belonged to phylogroup C ST23 group and carried the serotype 0176:H9. A plasmid-borne mcr-1.1 gene encoding for CR as well as a $a p h\left(3^{\prime}\right)$-Ia gene conferring resistance to neomycin were identified, both being co-located on the same IncHI2 plasmid. In addition, resistance genes conferring resistance to hygromycin B (aph(4)-Ia), sulfonamides (sul2), tetracyclines $($ tet $(A))$ and phenicols (floR and catA1), all antibiotics used in veterinary medicine, were evidenced. For both subjects, CR strains could not be retrieved in the initial stool of the subject or in the donor's stool. PCR experiments performed with specific primers to detect $m c r-1$ gene directly on the pre-therapeutic stool were also negative.

To our knowledge, this is the first report of the in-vivo selection of CR-Enterobacterales in the gut microbiota of patients after oral decontamination by colistin. The selection of CR strains (a naturally-resistant $H$. paralvei and a mcr-1 producing E. coli), both resistant to colistin and neomycin, may be the result either of the enrichment process by SOD of preexisting CR strains that had not been initially detected because of very low abundances, or of an exogenous acquisition, either from other individuals or through FMT. ${ }^{8}$ Indeed the transmission from FMT of MDR strains from positive donors is a potential risk. ${ }^{8}$ Despite our efforts to decrease the limit of detection of MCR producers by using a PCR technique directly on the pre-therapeutic stool sample and the donors' stools, we failed to detect the parental strain, either because CR strains were in intestinal niches, the limit of detection remained too high, or the strain was acquired exogenously. However, the mcr-1-positive E. coli is likely of animal origin according to its genetic features and its co-resistance profile. ${ }^{9}$ Indeed, phylogroup ST23 is frequently encountered among avian pathogenic E. coli (APEC) and co-resistances to many antibiotics used specifically in veterinary medicine is striking. ${ }^{10}$ Furthermore, the $\operatorname{aph}\left(3^{\prime}\right)$ Ia gene confers resistance to neomycin and paromomycin, the latter commonly used in cattle and pigs. The selection of the MCR-1 producer is an illustration of the "One Health" problem of resistance: a strain likely to have been selected by veterinary antibiotics among animals ended up in a patient's gut, later enriched by the use of colistin and neomycin as decontaminant. 


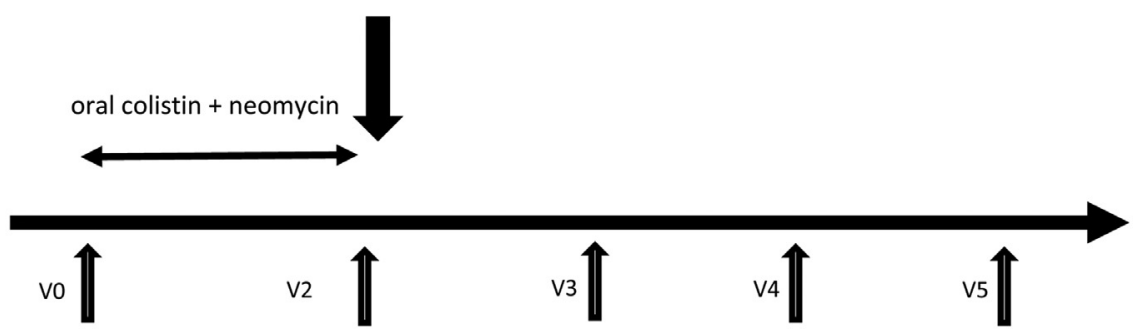

\begin{tabular}{|l|l|c|c|c|c|c|}
\hline & $\begin{array}{l}\text { Counts of Ent. } \\
\text { (CFU/g stool) }\end{array}$ & V0 & V2 & V3 & V5 \\
\hline Patient A & $\begin{array}{l}\text { Total } \\
\text { Enterobacterales }\end{array}$ & $10^{5}$ & $10^{4}$ & $10^{5}$ & $10^{5}$ & NA \\
\hline $\begin{array}{l}\text { colistin-R } \\
\text { Patient B }\end{array}$ & 0 & 0 & 0 & $\begin{array}{l}10^{1} \\
\text { (Hafnia paralvei) }\end{array}$ \\
$\begin{array}{l}\text { Total } \\
\text { Enterobacterales } \\
\text { colistin-R }\end{array}$ & $10^{5}$ & 0 & $10^{5}$ & $10^{5}$ & $10^{5}$ \\
\hline
\end{tabular}

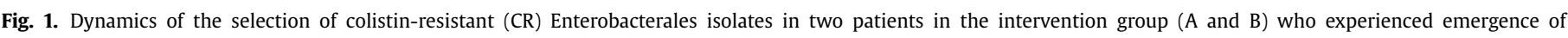

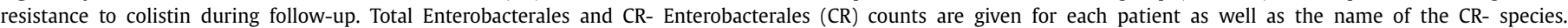

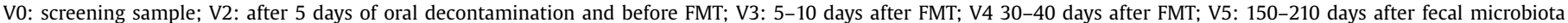
transplant (FMT). ${ }^{6} \mathrm{NA}$ : not available.

Although the small number of subjects is a clear limitation, this observation is a "proof-of-concept" of the risk of selection of CREnterobacterales after oral colistin treatment and FMT, at a time when colistin is one of the last resort antibiotics to treat MDREnterobacterales infections. The selection of commensal CR-E. coli is especially worrying, given the pathogenic potential of $E$. coli and its ability to widely colonize animals and humans. Given the controversial results of oral decontamination by colistin, we believe it should only be used with precautions and with thorough monitoring of CR.

\section{Acknowledgments}

We are indebted to all colleagues who contributed to this substudy in Paris and Geneva. In particular, we would like to thank the following members of the local R-Gnosis WP3 teams: Jacques Schrenzel, Gesuele Renzi and Naura Gamany.

This work was partially funded by the Laboratoire Européen Associé INSERM (Emerging Antibiotic Resistance in Gram-negative bacteria) and by the "Fondation pour la Recherche Médicale" (Equipe FRM 2016, grant number DEQ20161136698).

The 'RGNOSIS WP3' study was part of the European R-GNOSIS "Resistance in Gram-Negative Organisms: Studying Intervention Strategies" collaborative research project funded by the European Commission under the Seventh Framework Program (FP7/2007) for Research and technology (Grant Agreement no. 282512). Trial registration: ClinicalTrials.gov NCT02472600.

\section{Transparency declarations}

LP and PN have developed the SuperPolymyxin ${ }^{\circledR}$ screening medium. SH reports personal fees from Sandoz and DNA Electronics. All other authors declare no conflicts of interests.

\section{References}

1. Yan Z., Irene G., Ilias K., Jing L., Mon A.S., Jiayuan H., et al. Multifaceted mechanisms of colistin resistance revealed by genomic analysis of multidrug-resistant klebsiella pneumoniae isolates from individual patients before and after Colistin treatment. J Infect 2019;79(4):312-21. doi:10.1016/j.jinf.2019.07.009.

2. Jesús R.-B., Belén G.-G., Isabel M., Alvaro P.. Treatment of infections caused by extended-spectrum-beta-lactamase-, AMPC-, and carbapenemase-producing enterobacteriaceae. Clin Microbiol Rev 2018;31(2). doi:10.1128/CMR.00079-17.

3. Benedikt H., Thomas H., Ilker Uçkay, Gesuele R., Andrew S., Danièle S., et al. Decolonization of intestinal carriage of extended-spectrum $\beta$-lactamase-producing enterobacteriaceae with oral colistin and neomycin: a randomized, doubleblind, placebo-controlled trial. J Antimicrob Chemother 2013;68(10):2375-82. doi:10.1093/jac/dkt174.

4. de Smet A.M.G.A., Kluytmans J.A.J.W., Cooper B.S., Mascini E.M., Benus R.F.J., van der Werf T.S., et al. Decontamination of the digestive tract and oropharynx in ICU patients. N Engl J Med 2009;360(1):20-31. doi:10.1056/NEJMoa0800394.

5. Patrice N., Aurélie J., Laurent P.. A universal culture medium for screening polymyxin-resistant gram-negative isolates. J Clin Microbiol 2016;54(5):1395-9. doi:10.1128/JCM.00446-16.

6. Huttner Benedikt D., de Lastours V., Wassenberg M., Maharshak N., Mauris A. Galperine T., et al. A five-day course of oral antibiotics followed by faecal transplantation to eradicate carriage of multidrug-resistant enterobacteriaceae: a randomized clinical trial. Clin Microbiol Infect Off Publ Eur Soc Clin Microbiol Infect Dis 2019. doi:10.1016/j.cmi.2018.12.009.

7. Séverine B., Laurent P., Patrice N.. Real-time PCR for detection of plasmidmediated polymyxin resistance (MCR-1) from cultured bacteria and stools. J Antimicrob Chemother 2016;71(8):2318-20. doi:10.1093/jac/dkw139.

8. Zachariah D., Bloom P.P., Torres Soto M., Mansour M.K., Sater M.R.A., Huntley M.H., et al. Drug-Resistant E. coli bacteremia transmitted by fecal microbiota transplant. N Engl J Med 2019;381(21):2043-50. doi:10.1056/NEJMoa1910437.

9. Yi-Yun L., Yang W., Walsh T.R., Yi L.-X., Zhang R., Spencer J., et al. Emergence of plasmid-mediated Colistin resistance mechanism MCR-1 in animals and human beings in china: a microbiological and molecular biological study. Lancet Infect Dis 2016;16(2):161-8. doi:10.1016/S1473-3099(15)00424-7.

10. Guido C., Woodward M.J., Wu H., Alanazi M., Wallis T., La Ragione R.M.. Comparative genomics of European avian pathogenic E. coli (APEC). BMC Genomics 2016;17(1):960. doi:10.1186/s12864-016-3289-7.

Victoire de Lastours*

Université de Paris, IAME, INSERM, UMR 1137, F-75018 Paris, France Service de médecine interne, Hôpital Beaujon, AP-HP, F-92210 Clichy,

France 
Laurent Poirel

Université de Paris, IAME, INSERM, UMR 1137, F-75018 Paris, France Swiss National Reference Center for Emerging Antibiotic Resistance (NARA), University of Fribourg, Fribourg, Switzerland Emerging Antibiotic Resistance Unit, Medical and Molecular Microbiology, Department of Medicine, Faculty of Science and Medicine, Laboratoire Européen Associé "Emerging Antibiotic Resistance in Gram-negative bacteria ", INSERM, University of Fribourg, Fribourg, Switzerland

Benedikt Huttner, Stephan Harbarth Infection Control Program and WHO Collaborating Center, Geneva University Hospitals, Geneva, Switzerland Division of Infectious Diseases, Geneva University Hospitals, Geneva, Switzerland

Faculty of Medicine, University of Geneva, Geneva, Switzerland

Erick Denamur

Université de Paris, IAME, INSERM, UMR 1137, F-75018 Paris, France Laboratoire de Génétique Moléculaire, Hôpital Bichat, AP-HP, F-75018

Paris, France

Patrice Nordmann Université de Paris, IAME, INSERM, UMR 1137, F-75018 Paris, France Swiss National Reference Center for Emerging Antibiotic Resistance (NARA), University of Fribourg, Fribourg, Switzerland Emerging Antibiotic Resistance Unit, Medical and Molecular Microbiology, Department of Medicine, Faculty of Science and Medicine, Laboratoire Européen Associé " Emerging Antibiotic Resistance in Gram-negative bacteria ", INSERM, University of Fribourg, Fribourg, Switzerland

*Corresponding author at: Internal Medicine Department, Beaujon University Hospital, Assistance Publique Hôpitaux de Paris, 100 Boulevard Général Leclerc, 92100 Clichy, France. E-mail address: victoire.de-lastours@aphp.fr (V. de Lastours)

Accepted 8 January 2020 Available online 15 January 2020

https://doi.org/10.1016/j.jinf.2020.01.003

(C) 2020 The British Infection Association. Published by Elsevier Ltd. All rights reserved.

\section{Clinical significance of Pneumocystis jirovecii DNA detection by real-time PCR in hematological patient respiratory specimens}

\section{Dear Editor,}

We read with interest a recent paper in this Journal by Luzatti and colleagues, ${ }^{1}$ who explored the significance of the presence of Herpes simplex virus (HSV) DNA in lower respiratory tract (LRT) specimens for the diagnosis of HSV pneumonia in immunocompromised patients. The authors underlined the difficulty in gauging the clinical relevance of such a laboratory finding in the absence of histopathological data, as HSV shedding in the LRT may occur in the absence of disease. The interpretation of real-time PCR results for diagnosis of Pneumocystis jirovecii (PJ) pneumonia (PJP) faces an analogous challenge, since the presence of PJ DNA in LRT may reflect colonization (carriage) rather than infection. ${ }^{2}$ There is limited information on the clinical value of PJ real-time PCR in diagnosing
PJP in patients with hematological diseases: ${ }^{3-6}$ this is exceedingly challenging as the sensitivity of direct examination procedures is suboptimal due to low fungal burdens. ${ }^{3}$

Here, we report on our experience on this matter. A total of 219 episodes of pneumonia occurring in 192 consecutive patients with hematological disorders in which PJP was considered in the differential etiological diagnosis were included. Of these, 127 episodes developed in patients undergoing either allogeneic hematopoietic stem cell transplantation-allo-HSCT- $(n=86)$ or autologous-HSCT $(n=19)$, and 92 in non-transplant patients (acute leukemia, $n=61$; lymphoma, $n=16$; chronic leukemia, $n=8$; others, $n=2$ ). The patients were attended at the Hospital Clínico Universitario-HCU$(n=100)$ or at the Hospital Universitario Politécnico "La Fe" -HLF$(n=92)$ between June 2014 and August 2019. No patients in the cohort tested positive for HIV. This study was approved by the respective hospital ethics committee and informed consent was obtained from all patients.

A single specimen per episode was available for diagnosis (BAL fluids, $n=179$; sputa, $n=22$; TA, $n=17$ and bronchial biopsy, $n=1$ ). The RealCycler PJIR kit ${ }^{\circledR}$ (Progenie Molecular, Spain) was used at HCU, and the Pneumocystis jirovecii Real Time PCR Detection (CerTest Biotech; Zaragoza, Spain) was employed at HLF (see footnote in Table 1). Both assays target the large sub-unit of ribosomal (mtLSU) RNA gene. Preliminary experiments using 5 BAL specimens indicated that both assays yield comparable PCR cycle thresholds $\left(C_{\mathrm{T}} \mathrm{s}\right)$ (median, 28.2, range, 26.4-32.3 vs. median 27.5; range, 26.3-33.1, respectively; $P=0.89$ ).

All specimens tested negative by direct examination for PJ, whereas 27 were positive by real-time PCR (BAL, $n=18$; sputa, $n=7$, and TA, $n=2$ ); Following stringent clinical, microbiological and imaging criteria (Table 1), PJP was deemed to be the most probable diagnosis in 12 episodes occurring in unique patients. No histopathological confirmation of PJP was available for any patient.

PCR $C_{T}$ values inversely correlate with fungal burden in the sample. ${ }^{6}$ which is higher in patients with PJP than in colonized individuals. ${ }^{2}$ Here, overall, PJ PCR $C_{T} S$ in specimens from patients with PJP tended to be lower than in PJ carriers $(P=0.39)$; when only BAL fluid specimens were considered, the difference reached statistical significance (median, 29.0; range, 26.4-34.7 vs. median 34.6; range, $30.0-41.0 ; P=0.04)$. This finding is likely related to use of more standardized procedures for BAL fluid sampling. Receiver operating characteristic (ROC) curve analysis showed that a threshold $\mathrm{C}_{\mathrm{T}}$ value of 30.0 in BAL specimens displayed a sensitivity of $85.7 \%$ (95\% CI, $45.0-100 \%)$ and a specificity of $80 \%$ (95\% CI, $40.8-$ $100 \%$ ) for PJP diagnosis. A number of studies have established different $C_{T} s$ cut-offs for that purpose, ${ }^{6-9}$. In our view, however, the variability in the performance of different PCR assays and sampling conditions, heterogeneity of patient populations, and in particular the lack of a PJ international standard material for PCR result normalization precludes defining a consensus universal threshold nowadays.

The absence of anti-PJ prophylaxis, treatment with corticosteroids and serum LDH levels $\geq 400 \mathrm{U} / \mathrm{L}$ have been shown to be associated with PJP. ${ }^{3}$ Here, patients not undergoing anti-PJ prophylaxis were more likely to display a clinically significant PJ PCR result (Table 1). In turn, ROC curve analysis indicated that a cut-off LDH value $\geq 400 \mathrm{U} / \mathrm{L}$ had a sensitivity of $81.8 \%$ (CI 95\%, 59.0-100\%) and specificity of $67 \%$ (95\% CI, 34.0-99.3\%) for PJP diagnosis. In univariate regression logistic models, serum LDH values $\geq 400 \mathrm{U} / \mathrm{L}$ were associated with a clinically significant positive PCR PJ result (OR, 9.0; 95\% CI, 1.2-63.8; $P=0.02$ ). In contrast, corticosteroid use within the month before sampling was not different between 
Table 1

Characteristics of patients testing positive by real-time PCR for Pneumocystis jirovecii according to their final clinical diagnosis.

\begin{tabular}{|c|c|c|c|}
\hline \multirow[t]{2}{*}{ Parameter } & \multicolumn{2}{|c|}{ Pneumocystis jirovecii pneumonia ${ }^{a}$} & \multirow[t]{2}{*}{$P$ value $^{b}$} \\
\hline & Yes $(n=12)$ & No $(n=15)$ & \\
\hline \multicolumn{4}{|l|}{ Hematological disease in non-transplant patients } \\
\hline Acute myeloid leukemia & 3 & 3 & 0.97 \\
\hline Non-Hodgkin's lymphoma & 2 & 1 & \\
\hline Acute lymphocytic leukemia & 2 & 2 & \\
\hline Chronic lymphocytic leukemia & 1 & 1 & \\
\hline \multicolumn{4}{|l|}{ Type of hematopoietic stem cell transplantation } \\
\hline Allogeneic & 1 & 7 & 0.07 \\
\hline Autologous & 3 & 1 & \\
\hline \multicolumn{4}{|l|}{ Detection/recovery of other microorganisms in respiratory specimens } \\
\hline Yes & 3 & 14 & 0.001 \\
\hline No & 9 & 1 & \\
\hline \multicolumn{4}{|l|}{ Specimen type in which Pneumocystis jirovecii was detected ${ }^{c}$} \\
\hline Bronchoalveolar fluid & 6 & 12 & 0.45 \\
\hline Sputa & 5 & 2 & \\
\hline Tracheal aspirate & 1 & 1 & \\
\hline \multicolumn{4}{|l|}{ Antibiotic prophylaxis against Pneumocystis jirovecii $^{\mathrm{d}}$} \\
\hline Yes & 0 & 4 & 0.03 \\
\hline No & 12 & 11 & \\
\hline \multicolumn{4}{|l|}{ Corticosteroid use in the previous month to specimen sampling } \\
\hline Yes & 2 & 4 & 0.66 \\
\hline No & 10 & 11 & \\
\hline \multicolumn{4}{|l|}{ Oxygen therapy } \\
\hline Yes & 9 & 13 & 0.63 \\
\hline No & 3 & 2 & \\
\hline \multicolumn{4}{|l|}{ Exitus } \\
\hline Yes & $4^{e}$ & 8 & 0.44 \\
\hline No & 8 & 7 & \\
\hline
\end{tabular}

a The probability of Pneumocystis jirovecii (PJ) pneumonia (PJP) for each patient was retrospectively evaluated by an expert committee including infectious diseases and

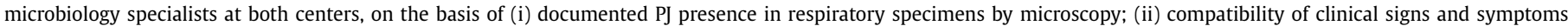

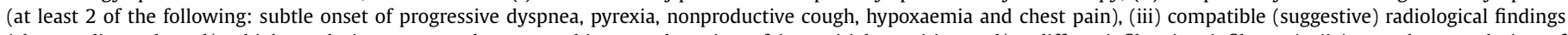

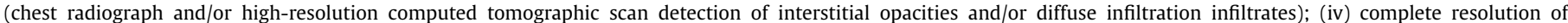

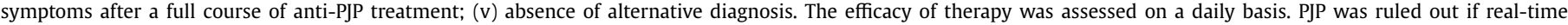

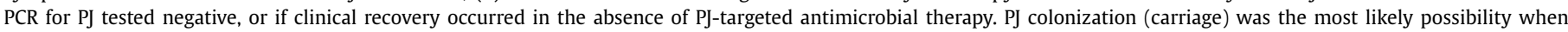
patients did not meet the above criteria and an alternate diagnosis was made.

b Frequencies were compared using the $\chi 2$ test (Fisher exact test) for categorical variables. Two-sided exact $P$ values were reported and $P$ values $\leq 0.05$ were considered statistically significant. The data were analyzed with the SPSS (version 20.0) statistical package.

c Respiratory tract specimens were obtained following conventional procedures. Specimens were examined for presence of ascus or trophic forms of PJ by microscopy

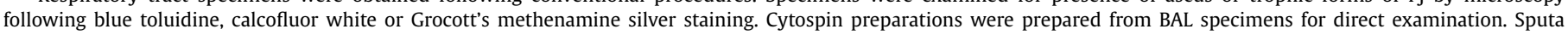

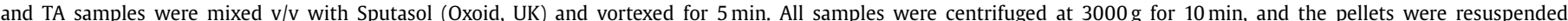

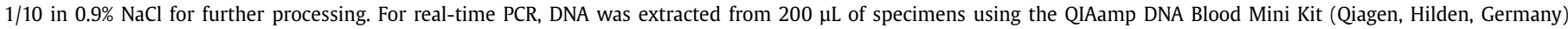

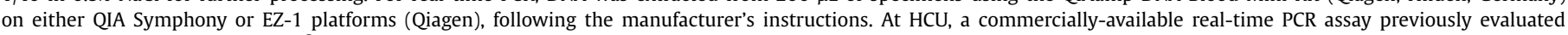

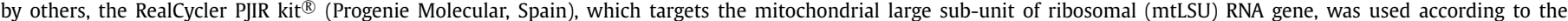

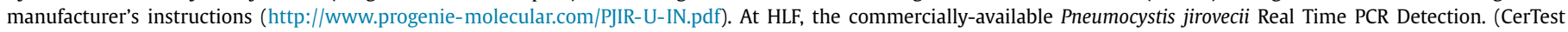

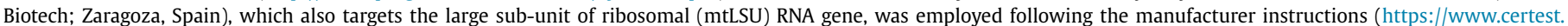

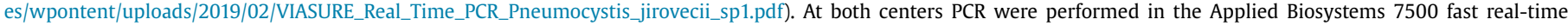

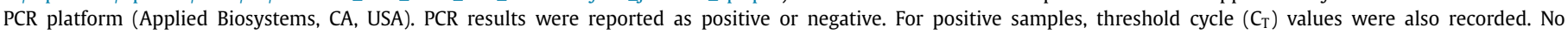
standard curve was generated with a positive control for quantitative estimations.

d Antimicrobial prophylaxis for PJP was performed with trimethoprim-sulfamethoxazole (TMP/SMX), one double-strength tablet (160 mg TMP/800 mg SMX) given 2 (in

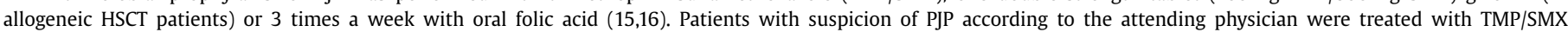
$15-20 \mathrm{mg} / \mathrm{kg}$ (TMP) $75-100 \mathrm{mg} / \mathrm{kg}$ (SMX) per day for 2-3 weeks.

Antimicrobial prophylaxis against PJ and of PJP occurrence

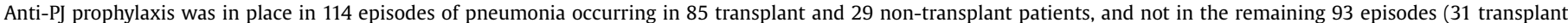

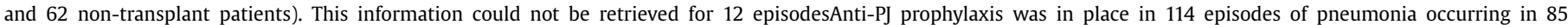

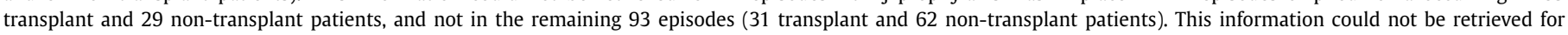
12 episodes.

e In all these cases, death was attributable to PJP.

patients with clinically significant PJ detection and PJ carriers (Table 1).

Detection or recovery of other microbial agents (one or more) was documented in 17 of the 27 specimens testing positive by PJ PCR (Table 2). In line with a previous report, ${ }^{9}$ this microbiological finding was significantly less frequent $(P=0.001)$ in specimens from patients with PJP than in colonized patients; in fact, microbial co-detection was inversely associated with PJP in univariate logistic regression models (OR, 0.024; 95\% CI, 0.002-0.26; $P=0.002$ ).

Strengths of the current study are the following: (i) clinical categorization of PJP was based upon stringent criteria defined by a multidisciplinary team; (ii) only hematological patients were included; (iii) a comprehensive routine investigation of microbial causes of pneumonia other than PJ was conducted; (iv) the experience of two centers was collected. In addition to its retrospective nature, our study also has some limitations: (i) we cannot completely rule out that some patients categorized as being PJ carriers did in fact have PJP, as most of these patients received full courses of TMP/SMX in combination with antimicrobials targeting other microbial agents. The lack of standardized criteria for PJP diagnosis makes clinical misclassification of patients a potential drawback in studies such as ours, particularly when no positive microscopy or histopathology findings are available; (ii) although we evaluated 
Table 2

Microbiological findings in patients testing positive for Pneumocystis jirovecii DNA by real-time PCR.

\begin{tabular}{|c|c|c|}
\hline Patient/clinical categorization & Specimen & Microorganisms detected or recovered ${ }^{\mathrm{a}}$ \\
\hline 1/PJP & BAL fluid & Respiratory syncitial virus type $\mathrm{B} /$ Candida albicans \\
\hline 2/PJP & BAL fluid & Escherichia coli \\
\hline 3/PJP & BAL fluid & Enterococus faecium \\
\hline 4/Carriage & Sputum & Rhinovirus \\
\hline 5/Carriage & BAL fluid & Aspergillus flavus \\
\hline 6/Carriage & BAL fluid & Pseudomonas aeruginosa \\
\hline 7/Carriage & BAL fluid & Coronavirus $229 \mathrm{e}$ \\
\hline 8/Carriage & Sputum & Parainfluenza virus type 3 \\
\hline 9/Carriage & BAL fluid & E. coli \\
\hline 10/Carriage & BAL fluid & Respiratory syncitial virus type $\mathrm{B} /$ Aspergillus fumigatus \\
\hline 11/Carriage & BAL fluid & Parainfluenza virus type 3 \\
\hline 12/Carriage & BAL fluid & Pseudomonas aeruginosa/Human metapneumovirus/Aspergillus fumigatus \\
\hline 13/Carriage & BAL fluid & Cytomegalovirus/Aspergillus fumigatus \\
\hline 14/Carriage & BAL fluid & Stenotrophomonas maltophilia/Human metapneumovirus \\
\hline 15/Carriage & BAL fluid & Aspergillus fumigatus \\
\hline 16/Carriage & TA & Parainfluenza virus type 3 \\
\hline 17/Carriage & TA & Parainfluenza virus type 3 \\
\hline
\end{tabular}

BAL, bronchoalveolar lavage; PJP, Pneumocysis jirovecii pneumonia; TA, tracheal aspirate.

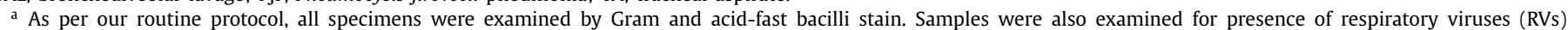

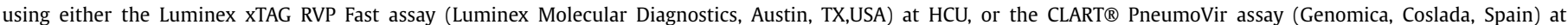

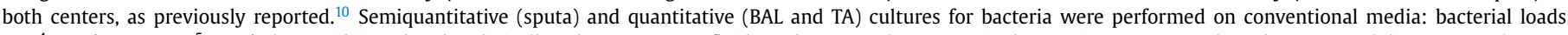

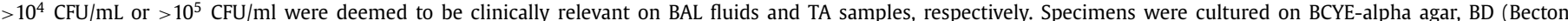

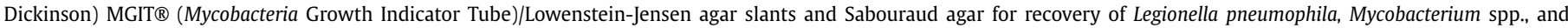

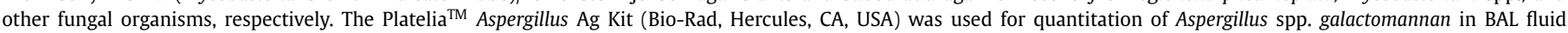

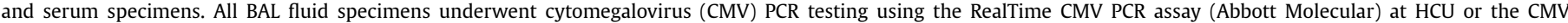
R-GENE® assay (Biomerieux) at HLF, as previously reported. ${ }^{10}$

over 200 patients, only 12 presumptively had PJP; (iii) two different commercially-available PCR assays were used across centers. Nevertheless, we found them to yield rather comparable $C_{T} s$.

In summary, we found that a positive PJ PCR result in respiratory specimens from transplant and non-transplant hematological patients with pneumonia frequently reflects colonization rather than infection; PCR $C_{T}$ values in BAL fluids, serum LDH levels and lack of co-detection of other microorganisms potentially involved may be helpful in clinical categorization in the absence of positive by PJ microcopy results.

\section{Declaration of Competing Interest}

We have no conflict of interest to declare.

\section{Acknowledgments}

No public or private funds were used for the current study. Eliseo Albert holds a Río Hortega research contract from the Carlos III Health Institute (Ref. CM18/00221). Estela Giménez holds a Juan Rodés research contract from the Carlos III Health Institute (Ref. JR18/00053).

\section{References}

1. Luzzati R., D’Agaro P., Busca A., Maurel C., Martellani F., Rosin C., et al. Herpes simplex virus (HSV) pneumonia in the non-ventilated immunocompromised host: burden and predictors. J Infect 2019;78:127-33.

2. Morris A., Norris K.A.. Colonization by Pneumocystis jirovecii and its role in disease. Clin Microbiol Rev 2012;25:297-317.

3. Alanio A., Hauser P.M., Lagrou K., Melchers W.J., Helweg-Larsen J., Matos O., et al. ECIL guidelines for the diagnosis of Pneumocystis jirovecii pneumonia in patients with haematological malignancies and stem cell transplant recipients. J Antimicrob Chemother 2016;71:2386-96.

4. Lu Y., Ling G., Qiang C., Ming Q., Wu C., Wang K., et al. PCR diagnosis of Pneumocystis pneumonia: a bivariate meta-analysis. J Clin Microbiol 2011:49:4361-3.

5. Fan L.C., Lu H.W., Cheng K.B., Li H.P., Xu J.F.. Evaluation of PCR in bronchoalveolar lavage fluid for diagnosis of Pneumocystis jirovecii pneumonia: a bivariate meta-analysis and systematic review. PLoS One 2013;8:e73099.

6. Guegan H., Robert-Gangneux F.. Molecular diagnosis of pneumocystis pneumonia in immunocompromised patients. Curr Opin Infect Dis 2019;32:314-21.
7. Alanio A., Desoubeaux G., Sarfati C., Hamane S., Bergeron A., Azoulay E., et al. Real-time PCR assay-based strategy for differentiation between active pneumocystis jirovecii pneumonia and colonization in immunocompromised patients. Clin Microbiol Infect 2011;17:1531-7.

8. Fauchier T., Hasseine L., Gari-Toussaint M., Casanova V., Marty P.M., Pomares C.. Detection of Pneumocystis jirovecii by quantitative PCR to differentiate colonization and pneumonia in immunocompromised HIV-Positive and HIV-Negative patients. J Clin Microbiol 2016;54:1487-95.

9. Robert-Gangneux F., Belaz S., Revest M., Tattevin P., Jouneau S., Decaux O., et al. Diagnosis of Pneumocystis jirovecii pneumonia in immunocompromised patients by real-time PCR: a 4-year prospective study. J Clin Microbiol 2014;52:3370-6.

10. Piñana J.L., Giménez E., Gómez M.D., Pérez A., González E.M., Vinuesa V., et al. Pulmonary cytomegalovirus (CMV) DNA shedding in allogeneic hematopoietic stem cell transplant recipients: implications for the diagnosis of CMV pneumonia. J Infect 2019;78:393-401.

José Luis Piñana ${ }^{1}$

Hematology Department, Hospital Universitari i Politècnic La Fe, Valencia, Spain

Eliseo Albert ${ }^{1}$

Microbiology Service, Hospital Clínico Universitario, Institute for Research INCLIVA, Valencia, Spain

María Dolores Gómez Microbiology Department, Hospital Universitari i Politècnic La Fe, Valencia, Spain

Ariadna Pérez, Juan Carlos Hernández-Boluda Hematology Service, Hospital Clínico Universitario, Institute for Research INCLIVA, Valencia, Spain

Juan Montoro

Hematology Department, Hospital Universitari i Politècnic La Fe, Valencia, Spain

Miguel Salavert

Department of Infectious Diseases, Hospital Universitari i Politècnic $\mathrm{La} \mathrm{Fe,} \mathrm{Valencia,} \mathrm{Spain}$

Eva María González

Microbiology Department, Hospital Universitari i Politècnic La Fe, Valencia, Spain 
Mar Tormo

Hematology Service, Hospital Clínico Universitario, Institute for Research INCLIVA, Valencia, Spain

Estela Giménez

Microbiology Service, Hospital Clínico Universitario, Institute for Research INCLIVA, Valencia, Spain

Marta Villalba, Aitana Balaguer-Roselló Hematology Department, Hospital Universitari i Politècnic La Fe, Valencia, Spain

Rafael Hernani

Hematology Service, Hospital Clínico Universitario, Institute for Research INCLIVA, Valencia, Spain

Felipe Bueno, Rafael Borrás Microbiology Service, Hospital Clínico Universitario, Institute for Research INCLIVA, Valencia, Spain

Jaime Sanz

Hematology Department, Hospital Universitari i Politècnic La Fe, Valencia, Spain

Carlos Solano

Hematology Service, Hospital Clínico Universitario, Institute for Research INCLIVA, Valencia, Spain

Department of Medicine, School of Medicine, University of Valencia,

Valencia, Spain

David Navarro*

Hematology Service, Hospital Clínico Universitario, Institute for Research INCLIVA, Valencia, Spain Department of Microbiology, School of Medicine, University of Valencia, Valencia, Spain

*Corresponding author at: Department of Microbiology, School of Medicine, University of Valencia, Av. Blasco Ibáñez 17, 46010 Valencia, Spain. E-mail address: david.navarro@uv.es (D. Navarro)

${ }^{1}$ Both authors contributed equally to the present work. Accepted 4 January 2020 Available online 15 January 2020

https://doi.org/10.1016/j.jinf.2020.01.001

(C) 2020 The British Infection Association. Published by Elsevier Ltd. All rights reserved.

\section{Epidemiology and case-control study of Lassa fever outbreak in Nigeria from 2018 to 2019}

\section{Dear Editor}

Poller et al., in this Journal, provided a useful consensus for use of personal protective equipment for managing high consequence infectious disease ${ }^{1}$. Although this was driven largely by recent Ebola virus disease emergencies, we should remind your readers of the continuing problem of Lassa fever (LF) in West Africa. LF is a febrile infectious disease caused by Lassa virus. The clinical presentation of the disease is nonspecific and includes fever, fatigue, hemorrhage, gastrointestinal symptoms, respiratory symptoms, and neurological symptoms ${ }^{2}$. The observed case fatality rate among patients hospitalized with severe LF is $15-20 \%^{3,4}$. The disease is mainly spread to humans through contamination with the urine or faeces of infected rats $^{2}$. Human-to-human transmission can occur through contact with the body fluids of infected per- sons. Therefore, health care workers are at high risk for infection when the standard precautions for infection prevention and control including appropriate personal protective equipment are inadequate ${ }^{5}$.

It is estimated that there are approximately 300,000 LF cases annually, resulting in approximately 5000 deaths in West African countries $^{4}$. In 2018, Nigeria had a large LF outbreak, and we previously reported epidemiological characteristics of the outbreak, analyzing data collected between January and May 2018. ${ }^{6}$ However, information on laboratory-negative suspected cases was not enough to conduct a case-control study to fully determine the risk factors and clinical characteristics of the disease. Nigeria had a LF outbreak in 2019 as well. ${ }^{7}$ Here we report the epidemiological and clinical characteristics of the outbreak including case-control analysis against laboratory-negative suspected cases using data collected between 1st January 2018 and 6th October 2019.

From January to December 2018, there were 3498 suspected cases, including 633 laboratory-confirmed LF cases. In 2019, there were 4019 suspected cases reported by 6th October, including 721 laboratory-confirmed LF cases. Details on the case definition, laboratory test, surveillance, and data collection have been described previously. ${ }^{6}$ Of the confirmed LF cases, there were $171 \mathrm{fa}$ talities (case fatality rate, 27.0\%) in 2018 and 154 fatalities (case fatality rate, $21.4 \%$ ) in 2019 . The number of laboratory-confirmed LF cases and positivity rate peaked in the dry season (JanuaryMarch) in both 2018 and 2019 (Fig. 1(A)). The largest number of laboratory-confirmed LF cases were reported from the neighboring Edo and Ondo states in both 2018 and 2019 (Fig. 1(B)). There were laboratory-confirmed LF cases in states such as Kebbi and Zamfara that had no reported cases previously, in 2019.

During the study period, the detailed demographic and clinical information was collected for 1305 laboratory-confirmed LF cases (of 1354 cases, 96.4\%) and 3350 laboratory-negative suspected cases (of 6163 cases, 54.4\%). Chi-square tests were conducted to compare the distribution of age, sex, and each symptom between the laboratory-confirmed LF cases and laboratory-negative suspected cases (Table 1). The proportion of children was significantly lower in laboratory-confirmed LF cases compared with that in laboratory-negative suspected cases. The proportion of males was significantly higher in laboratory-confirmed LF cases than that in laboratory-negative suspected cases.

Fever was the most prevalent symptom in both laboratoryconfirmed LF cases and laboratory-negative suspected cases, followed by headache (Table 1). Gastrointestinal symptoms, such as abdominal pain, vomiting, and diarrhea, were observed in more than $30 \%$ of laboratory-confirmed LF cases, whereas hemorrhaging was observed in $21.1 \%$ of laboratory-confirmed LF cases. While the prevalence of face/neck edema was low even in laboratoryconfirmed LF cases (6.4\%), nonetheless, the odds ratio of having face/neck edema was 6.0 times high for laboratory-confirmed LF cases.

We here reported the LF outbreak in 2018-2019 largest recorded in history. While previous studies have focused on laboratory-confirmed LF cases and mainly compared fatal cases and survived cases, $, 6,8,9$ our observation revealed the difference between laboratory-confirmed LF cases and laboratory-negative suspected cases. The age and sex distribution differed significantly between laboratory-confirmed LF cases and laboratory-negative suspected cases. Fever, headache, and gastrointestinal symptoms were the most common symptoms in laboratory-confirmed LF cases, which are similar to those reported previously. ${ }^{6,8}$ However, these symptoms were also prevalent in laboratory-negative suspected cases. Clinical guidelines for LF state that edema in the face and neck is a specific sign of the disease. ${ }^{10}$ The present study found that the symptom had a significantly high odds ratio for 

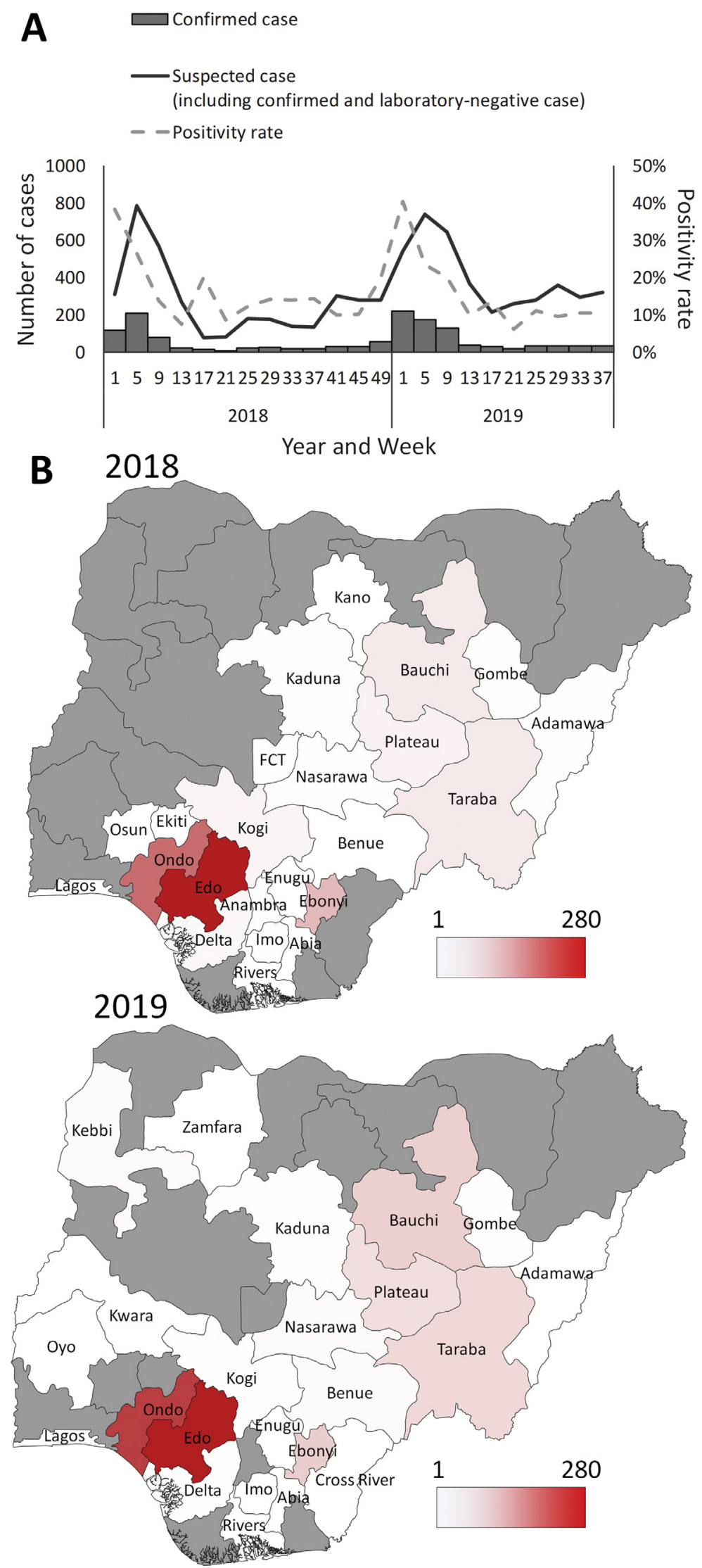

Fig. 1. Temporal and geographical distribution of LF cases in Nigeria from 1st January 2018 to 6th October 2019

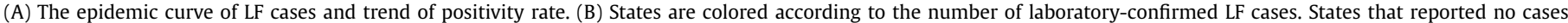
are shaded in gray. 
Table 1

Age, sex, and symptoms distribution in laboratory-confirmed LF cases and laboratory-negative suspected cases.

\begin{tabular}{|c|c|c|c|c|c|}
\hline Category & Variable & $\begin{array}{l}\text { Laboratory-confirmed } \\
\text { LF case }(\%)\end{array}$ & $\begin{array}{l}\text { Laboratory-negative } \\
\text { suspected case (\%) }\end{array}$ & Odds ratio*1 & $95 \%$ confidence interval $^{* 1}$ \\
\hline \multirow[t]{7}{*}{ Age group (years) } & $0-9$ & $112 / 1243(9.0 \%)$ & $581 / 3191(18.2 \%)$ & 0.45 & $0.36-0.55$ \\
\hline & $10-19$ & $179 / 1243(14.4 \%)$ & $478 / 3191(15.0 \%)$ & 0.96 & $0.79-1.2$ \\
\hline & $20-29$ & $280 / 1243(22.5 \%)$ & $693 / 3191(21.7 \%)$ & 1.0 & $0.90-1.2$ \\
\hline & $30-39$ & $294 / 1243(23.7 \%)$ & $603 / 3191(18.9 \%)$ & 1.3 & $1.1-1.6$ \\
\hline & $40-49$ & $171 / 1243(13.8 \%)$ & $346 / 3191(10.8 \%)$ & 1.3 & $1.1-1.6$ \\
\hline & $50-59$ & $108 / 1243(8.7 \%)$ & $223 / 3191(7.0 \%)$ & 1.3 & $1.0-1.6$ \\
\hline & $60-$ & $99 / 1243(8.0 \%)$ & $267 / 3191(8.4 \%)$ & 0.95 & $0.75-1.2$ \\
\hline \multirow[t]{2}{*}{ Sex } & Female & $542 / 1274(42.5 \%)$ & $1596 / 3244(49.2 \%)$ & 0.76 & $0.67-0.87$ \\
\hline & Male & $732 / 1274(57.5 \%)$ & $1648 / 3244(50.8 \%)$ & 1.3 & $1.1-1.5$ \\
\hline \multirow[t]{16}{*}{ Symptom } & Fever & $971 / 1019(95.3 \%)$ & $2112 / 2284(92.5 \%)$ & 1.6 & $1.2-2.3$ \\
\hline & Headache & $550 / 829(66.3 \%)$ & $1074 / 1719(62.5 \%)$ & 1.2 & $1.0-1.4$ \\
\hline & Fatigue & $443 / 746$ (59.4\%) & $391 / 1321(29.6 \%)$ & 3.5 & $2.9-4.2$ \\
\hline & Abdominal pain & $425 / 776(54.8 \%)$ & $545 / 1353(40.3 \%)$ & 1.8 & $1.5-2.1$ \\
\hline & Vomiting & $388 / 741(52.4 \%)$ & $302 / 1237(24.4 \%)$ & 3.4 & $2.8-4.1$ \\
\hline & Anorexia & $276 / 666(41.4 \%)$ & $188 / 1212(15.5 \%)$ & 3.9 & $3.1-4.8$ \\
\hline & Cough & $221 / 661$ (33.4\%) & $223 / 1221(18.3 \%)$ & 2.2 & $1.8-2.8$ \\
\hline & Diarrhea & $233 / 697$ (33.4\%) & $159 / 1204(13.2 \%)$ & 3.3 & $2.6-4.2$ \\
\hline & Nausea & $187 / 648(28.9 \%)$ & $89 / 1164(7.6 \%)$ & 4.9 & $3.7-6.5$ \\
\hline & Chest pain & $168 / 627$ (26.8\%) & $91 / 1145$ (7.9\%) & 4.2 & $3.2-5.6$ \\
\hline & Muscle pain & $161 / 635(25.4 \%)$ & $87 / 1162(7.5 \%)$ & 4.2 & $3.2-5.6$ \\
\hline & Hemorrhaging & $132 / 625(21.1 \%)$ & $131 / 1145(11.4 \%)$ & 2.1 & $1.6-2.7$ \\
\hline & Joint pain & $102 / 611(16.7 \%)$ & $52 / 1137(4.6 \%)$ & 4.2 & $2.9-5.9$ \\
\hline & Difficulty breathing & $84 / 609$ (13.8\%) & $77 / 1140(6.8 \%)$ & 2.2 & $1.6-3.1$ \\
\hline & Altered state of consciousness & $54 / 604(8.9 \%)$ & $34 / 1132(3.0 \%)$ & 3.2 & $2.0-4.9$ \\
\hline & Face/neck edema & $17 / 267(6.4 \%)$ & $10 / 896(1.1 \%)$ & 6.0 & $2.7-13.3$ \\
\hline Total $^{* 2}$ & & 1305 & 3350 & Not applicable & Not applicable \\
\hline
\end{tabular}

*1 Odds ratios for laboratory-confirmed LF cases were shown. 95\% confidence intervals were calculated using Chi-square test with Mantel-Haenszel common odds ratio estimate.

*2 Denominator varies for each variable because information on the age, sex, and/or symptom was missing in some cases.

confirmed LF although the prevalence of this symptom was low. Unfortunately, we did not determine the differential diagnosis for the laboratory-negative suspected cases. Laboratory tests for the differential diagnoses are now underway for the LF-negative samples collected during the outbreak. The results would provide us further insight for better clinical management of patients with febrile illnesses in LF-endemic areas.

In addition to the standard precautions for infection prevention and control including appropriate personal protective equipment pointed out by Poller et al., ${ }^{1}$ it is important to know epidemiological and clinical characteristics of high consequence infectious diseases such as LF. That would help healthcare workers and public health officers increase an index of suspicion of the diseases, further leading to better clinical management and surveillance.

\section{Declaration of Competing Interest}

The authors have declared that no conflicts of interest exist.

\section{Funding}

This work was partially supported by the Leading Initiative for Excellent Young Researchers from the Ministry of Education, Culture, Sport, Science \& Technology of Japan and the Japan Society for the Promotion of Science (grant number, 16809810). The funders had no role in study design, data collection and analysis, decision to publish, or preparation of the manuscript.

\section{Acknowledgments}

We thank all the staff of the domestic and international organizations who fought against this outbreak, including those at the various health care facilities, Lassa fever diagnostic laborato- ries, Nigeria Centre for Disease Control, World Health Organization, African Field Epidemiology Network, Public Health England, eHealth Africa, Pro Health International, University of Maryland Baltimore, US Centers for Disease Control and Prevention, Alliance for International Medical Action, Médecins Sans Frontières, and numerous other partners. We also express our sincerest condolences to the families and friends of those who died during the outbreak.

\section{References}

1. Bozena P., Anne T., Samantha H., Mike B., Mike J., Erica P., et al. A unified personal protective equipment ensemble for clinical response to possible high consequence infectious diseases: a consensus document on behalf of the HCID programme. J Infect 2018. doi:10.1016/j.jinf.2018.08.016.

2. Kay R.J., Baglole Deborah J.. Lassa fever: epidemiology, clinical features, and social consequences. BMJ Br Med J 2003;327(7426):1271-5. doi:10.1136/bmj.327. 7426.1271 .

3. World Health Organization. Lassa fever fact sheet. Available at http://origin.who. int/mediacentre/factsheets/fs179/en/. Accessed May 13, 2018, 2017.

4. Centers for Disease Control and Prevention. Lassa Fever. Available at http:// www.cdc.gov/vhf/lassa/. Accessed May 13, 2018, 2015.

5. Fisher-Hoch S.P., Tomori O., Nasidi A., Perez-Oronoz G.I., Fakile Y., Hutwagner L., et al. Review of cases of nosocomial Lassa fever in Nigeria: the high price of poor medical practice. BMJ 1995;311(7009):857. doi:10.1136/bmj.311.7009.857.

6. Ilori Elsie A., Yuki F., Ipadeola Oladipupo B., Dan-Nwafor Chioma C., Anwar A. Womi-Eteng Oboma E., et al. Epidemiologic and clinical features of Lassa fever outbreak in Nigeria, January 1-May 6, 2018. Emerg Infect Dis 2019;25(6):106674. doi:10.3201/eid2506.181035.

7. Dan-Nwafor Chioma C., Yuki F., Ilori Elsie A., Oladipupo I., Akabike Kachikwulu O., Anthony A., et al. Measures to control protracted large Lassa fever outbreak in Nigeria, 1 January to 28 April 2019. Euro Surveill 2019;24(20). doi:10.2807/1560-7917.ES.2019.24.20.1900272.

8. Peter O., Andres C., Chukwuemeka A., Christopher I., Omoregie O., Shervin T. et al. Clinical and laboratory predictors of Lassa fever outcome in a dedicated treatment facility in Nigeria: a retrospective, observational cohort study. Lancet Infect Dis 2018, doi:10.1016/S1473-3099(18)30121-X.

9. Asogun Danny A., Adomeh Donatus I., Jacqueline E., Ikponmwonsa O., Meike H., Martin G., et al. Molecular diagnostics for Lassa fever at Irrua specialist teaching hospital, Nigeria: lessons learnt from two years of laboratory operation. PLoS Negl Trop Dis 2012. doi:10.1371/journal.pntd.0001839.

10. Nigeria Centre for Disease Control. National guidelines for Lassa fever case management. Available at https://ncdc.gov.ng/themes/common/docs/protocols/ 92 1547068532.pdf. Accessed March 25, 2019, 2018. 
Oladipupo Ipadeola ${ }^{1}$

US Centers for Disease Control and Prevention, Plot 1075, Diplomatic Drive Central Business Area, Abuja, Nigeria

Yuki Furuse*1

World Health Organization, Plot 617/618, Central Area District, PMB 2861, Abuja, Nigeria

Institute for Frontier Life and Medical Sciences, Kyoto University, 53

Shogoin Kawaracho, Sakyo-ku, Kyoto, Japan

Hakubi Center for Advanced Research, Kyoto University, Yoshida Honmachi, Sakyo-ku, Kyoto, Japan

Elsie A. Ilori, Chioma C. Dan-Nwafor, Kachikwulu O. Akabike, Anthony Ahumibe

Nigeria Centre for Disease Control, Plot 801, Ebitu Ukiwe Street, Jabi, Abuja, Nigeria

Winifred Ukponu

University of Maryland Baltimore, Plot 801, Ebitu Ukiwe Street, Jabi, Abuja, Nigeria

Georgetown University, Plot 801, Ebitu Ukiwe Street, Jabi, Abuja, Nigeria

Lawal Bakare, Gbenga Joseph Nigeria Centre for Disease Control, Plot 801, Ebitu Ukiwe Street, Jabi, Abuja, Nigeria

Muhammad Saleh

US Centers for Disease Control and Prevention, Plot 1075, Diplomatic Drive Central Business Area, Abuja, Nigeria

Esther Namukose Muwanguzi, Adebola Olayinka, Geoffrey Namara, Dhamari Naidoo

World Health Organization, Plot 617/618, Central Area District, PMB 2861, Abuja, Nigeria

Akanimo Iniobong, Michael Amedu Nigeria Centre for Disease Control, Plot 801, Ebitu Ukiwe Street, Jabi, Abuja, Nigeria

Nkem Ugbogulu

Pro Health International, 49 Kado District, Cadastral Zone B09 Kado, Abuja, Nigeria

Favour Makava

University of Maryland Baltimore, Plot 801, Ebitu Ukiwe Street, Jabi, Abuja, Nigeria

Olawunmi Adeoye, Chukwuemeka Uzoho Helmholtz Centre for Infection Research, Plot 801, Ebitu Ukiwe Street, Jabi, Abuja, Nigeria

Chimezie Anueyiagu, Tochi J. Okwor, Nwando G. Mba, Adejoke Akano, Abiodun Ogunniyi, Amina Mohammed Nigeria Centre for Disease Control, Plot 801, Ebitu Ukiwe Street, Jabi, Abuja, Nigeria

Ayodele Adeyemo eHealth Africa, 4-6 Independence Road, Kano, Nigeria

Dike K. Ugochukwu, Emmanuel Agogo, Chikwe Ihekweazu* Nigeria Centre for Disease Control, Plot 801, Ebitu Ukiwe Street, Jabi, Abuja, Nigeria

*Corresponding author at: Institute for Frontier Life and Medical Sciences, Kyoto University, 53 Shogoin Kawaracho, Sakyo-ku, Kyoto, Japan.

E-mail addresses: furusey.kyoto@gmail.com (Y. Furuse), chikwe.ihekweazu@ncdc.gov.ng (C. Ihekweazu)
1 These authors contributed equally to this article. Accepted 31 December 2019 Available online 15 January 2020

https://doi.org/10.1016/j.jinf.2019.12.020

(c) 2020 The British Infection Association. Published by Elsevier Ltd. All rights reserved.

\section{Human pseudorabies virus infection: A new threat in China}

Dear Editor,

Recently, several studies in this journal have demonstrated the threat of animal-derived viruses to humans. ${ }^{1-3}$ Since 2018, an increase in human pseudorabies virus (PRV) infection cases has been reported in China, indicating a new animal-derived virus threat to human health.

Porcine pseudorabies (PR), also known as Aujeszky's disease, is one of the most economically important viral diseases in pigs globally. Its causative agent is PRV, which is classified into the genus Varicellovirus of subfamily Alphaherpesvirinae, family Herpesviridae. PRV is almost always fatal in newborn piglets, is frequently accompanied by neurological symptoms, and may cause abortions and/or stillbirths in pregnant sows. PRV primarily infects members of the Suidae family and can also infect other domestic and wild mammals, including horses, cattle, sheep, goats, dogs, cats, etc. Currently, vaccination is the most effective strategy for PR prevention and control in pigs worldwide.

In China, PRV infections in pigs were first recorded in 1947. In the 1970s, an inactivated vaccine consisting of the Bartha-K61 vaccine strain was imported into China. Since then, this vaccine has been widely used in pig vaccination for PR prevention and control. Before 2011, no large PR outbreaks were reported in pigs in China. However, after late 2011, novel PRV wild-type variants emerged in nearly all regions of China and affected a number of swine herds vaccinated regularly with the Bartha-K61 vaccine, resulting in significant economic losses. ${ }^{4}$ Subsequent animal experiments indicated that the Bartha-K61 vaccine could not provide complete protection for pigs against a challenge with novel PRV wild-type variants in China. For control and eradication of PR, the disease was listed in the "Mid- and Long-term Animal Disease Prevention and Control Program in China (2012-2020)" by the Chinese government, with the aim of eliminating PR in China by 2020 (http://www.gov.cn/zwgk/2012-05/25/content_2145581.htm). However, vaccination for PRV is still voluntary and not required in China. A nationwide epidemiological investigation in 2014 demonstrated a high prevalence of $23.26 \%$ of PRV among swine herds in China. $^{5}$

Humans were previously regarded as refractory for PRV infection, although serological PRV antibody positivity was found in three cases. In 2018, the first human PRV infection case with direct molecular evidence was reported in China (Case 1, Table 1 ). ${ }^{6}$ In this case, the eyes of a 46-year-old woman were directly exposed to sewage on a hog farm. In the following two weeks, symptoms of fever, headache, coma, and endophthalmitis were observed in the patient. Next-generation sequencing (NGS) indicated that PRV DNA was detected in her vitreous humor samples but not in her cerebrospinal fluid (CSF). After surgery, the patient was discharged, but her vision remained impaired. In a subsequent study, Zhao et al. 


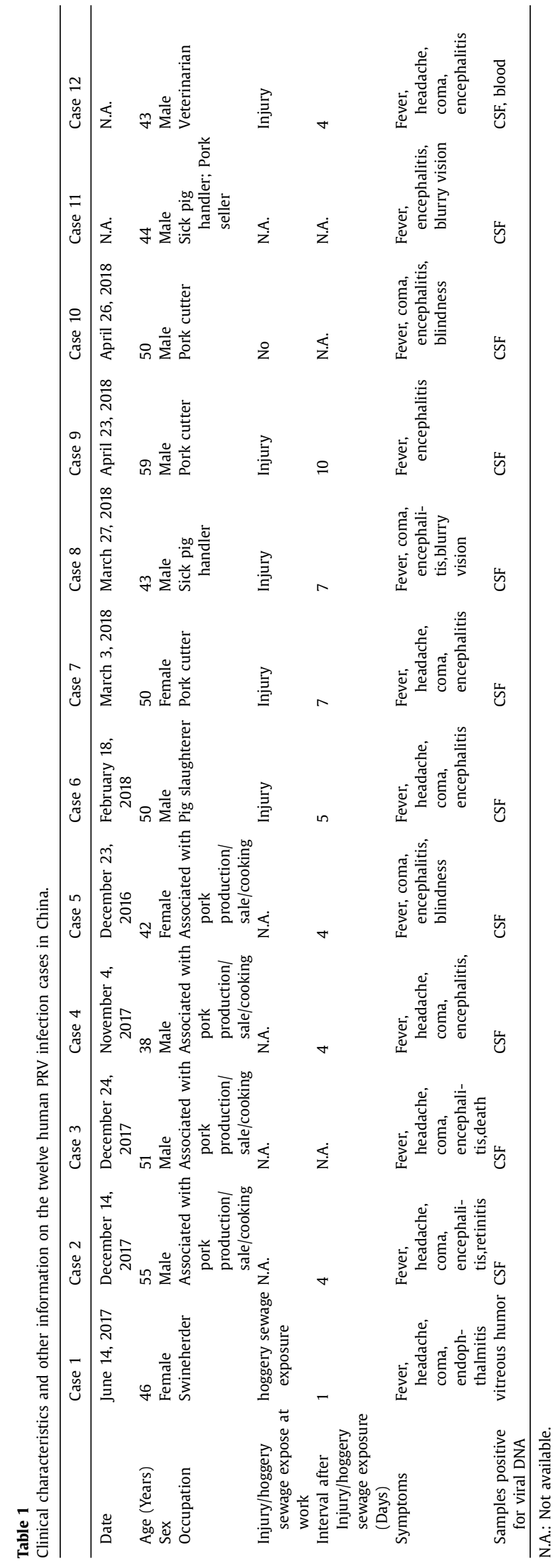

analyzed CSF samples from four patients with encephalitis of unknown etiology using NGS (Cases 2-5, Table 1) and found molecular evidence of PRV infection. ${ }^{7}$ In addition, retinitis and blindness was observed in two cases (Cases 2, 5, Table 1), and the patient in Case 3 died. The occupation of the four patients was all associated with pork production/sale/cooking. In 2019, six other human PRV infection cases involving encephalitis were reported in China, and all patients were pork/pig handlers or veterinarians..$^{8-10}$ It was noted that all patients still suffered from various sequelae after discharge, except for in one case where the patient died.

Increasing reports on human PRV infection cases in China have recently indicated that PRV poses a significant threat to public health in China, especially in people in close contact with sick pigs and/or related pork products/contaminants. To reduce the risk of PRV infection in susceptible workers, it is necessary to promulgate relevant policies by the Chinese government to promote PR vaccine development to protect pigs from infection with novel PRV wild-type variants currently circulating in China. In addition, relevant policies should be updated by the Chinese government to monitor vaccination status and virus variation in pigs nationwide. Moreover, it seems that PRV can infect humans via injury to the skin or eyes. Until now, no effective drugs to prevent the progression of the disease caused by PRV infection have been reported. Therefore, it is necessary to improve biosafety and self-protection awareness in susceptible populations that have contact with sick pigs and work in jobs related to handling pork products/contaminants. Promoting drug development for curing PRV-related disease in infected patients may also help reduce the currently increasing threat of PRV to human health in China.

\section{Declaration of Competing Interest}

All authors declare that they have no competing interests.

\section{Acknowledgments}

This work was supported by the Science and Technology Project in Guangdong Province of China (2015B020203006) and the Industry Technology System of Modern Agriculture Construction Fund (CARS-35).

\section{References}

1. Kang Y.F., Shen X.J., Yuan R.Y., Xiang B., Fang Z.X., Murphy R.W., et al. Pathogenicity and transmissibility of three avian influenza a (H5N6) viruses isolated from wild birds. J Infect 2018;76:286-94.

2. Tong X.C., Weng S.S., Xue F., Wu X., Xu T.M., Zhang W.H.. First human infection by a novel avian influenza A(H7N4) virus. J Infect 2018;77:249-57.

3. Yang Y., Wong G., Yang L., Tan S., Li J., Bai B., et al. Comparison between human infections caused by highly and low pathogenic H7N9 avian influenza viruses in Wave Five: clinical and virological findings. J Infect 2019;78:241-8.

4. Yu X., Zhou Z., Hu D., Zhang Q., Han T., Li X., et al. Pathogenic pseudorabies virus, China, 2012. Emerging Infect Dis 2014;20:102-4

5. Liu Y., Zhang S., Xu O., Wu J., Zhai X. Li S., et al. Investigation on pseudorabies prevalence in Chinese swine breeding farms in 2013-2016. Trop Anim Health Prod 2018;50:1279-85.

6. Ai J.W., Weng S.S., Cheng Q., Cui P., Li Y.J., Wu H.L., et al. Human endophthalmitis caused by pseudorabies virus infection, China, 2017. Emerging Infect Dis 2018;24:1087-90.

7. Zhao W.L., Wu Y.H., Li H.F., Li S.Y., Fan S.Y., Wu H.L., et al. Clinical experience and next-generation sequencing analysis of encephalitis caused by pseudorabies. Natl Med J China 2018;98:1152-7 (in Chinese).

8. Yang X., Guan H., Li C., Li Y., Wang S., Zhao X., et al. Characteristics of human encephalitis caused by pseudorabies virus: a case series study. Int J Infect Dis Official Publ Int Soc Infect Dis 2019;87:92-9.

9. Wang Y., Nian H., Li Z., Wang W., Wang X., Cui Y.. Human encephalitis complicated with bilateral acute retinal necrosis associated with pseudorabies virus infection: a case report. Int I Infect Dis Official Publ Int Soc Infect Dis 2019;89:51-4.

10. Yang $H_{\text {., }}$ Han H., Wang H., Cui Y., Liu H., Ding S.. A case of human viral encephalitis caused by pseudorabies virus infection in China. Front Neurol 2019;10:534 
Jiajun $\mathrm{Ou}$

College of Veterinary Medicine, South China Agricultural University, Guangzhou 510642, Guangdong Province, People's Republic of China Guangdong Provincial Key Laboratory of Prevention and Control for Severe Clinical Animal Diseases, Guangzhou 510642, Guangdong Province, People's Republic of China Guangdong Technological Engineering Research Center for Pet, Guangzhou 510642, Guangdong Province, People's Republic of China

Siqi Cai College of Veterinary Medicine, South China Agricultural University, Guangzhou 510642, Guangdong Province, People's Republic of China

Feiyan Zheng, Gang Lu* College of Veterinary Medicine, South China Agricultural University, Guangzhou 510642, Guangdong Province, People's Republic of China Guangdong Provincial Key Laboratory of Prevention and Control for Severe Clinical Animal Diseases, Guangzhou 510642, Guangdong Province, People's Republic of China

Guangdong Technological Engineering Research Center for Pet, Guangzhou 510642, Guangdong Province, People's Republic of China

Guihong Zhang* College of Veterinary Medicine, South China Agricultural University, Guangzhou 510642, Guangdong Province, People's Republic of China

*Corresponding authors at: College of Veterinary Medicine, South China Agricultural University, Guangzhou 510642, Guangdong Province, People's Republic of China.

E-mail addresses: LG@scau.edu.cn (G. Lu), guihongzh@scau.edu.cn (G. Zhang)

Accepted 27 December 2019 Available online 15 January 2020

https://doi.org/10.1016/j.jinf.2019.12.018

(C) 2020 The British Infection Association. Published by Elsevier Ltd. All rights reserved.

\section{Detection of mobile colistin resistance gene $\mathrm{mcr}-9$ in carbapenem-resistant Klebsiella pneumoniae strains of human origin in Europe}

\section{Dear Editor}

The emergence and spread of Gram-negative bacteria, for example, Klebsiella pneumoniae, co-producing carbapenemases and mobilized colistin resistance ( $\mathrm{mcr}$ ) genes limit our choice for treating multidrug-resistant infections, posing significant threats to public health. Herein, we reported the discovery of mcr-9 gene in 28 K. pneumoniae strains isolated from patients in eight European countries, including Belgium $(n=2)$, Denmark $(n=1)$, Montenegro $(n=13)$, Poland $(n=1)$, Romania $(n=1)$, Serbia $(n=1)$, Slovenia $(n=1)$, and Spain $(n=7)$. Notably, the co-existence of mcr-9 and the carbapenemase-encoding genes, NDM-1, VIM-1, and OXA-48 were confirmed in K. pneumoniae isolates of human origin. Phylogenetic analysis suggested that mcr-9-carrying $K$. pneumoniae isolates, including 23 carbapenem-resistant and five susceptible K. pneumoniae strains, show a highly geographically clustered pattern. Genetic environment analysis revealed the presence of insertion element IS903, IS1 or a cupin fold metalloprotein, wbuC, in the mcr-9 flanking. Taken together, these findings indicated that $m c r-9$ has existed for a long time and already spread among CRKP isolates of human origin in Europe since 2013, further increasing the significant threat of public health through either the nosocomial spread or environmental routes.
The mobilized colistin resistance ( $m c r$ ) gene $m c r-9$ was detected in 28 human gut microbiomes, which has been disseminating across three continents, including Asia, Europe and America, recently published in the Journal of Infection. ${ }^{1}$ The rapid increase in carbapenem resistance among Gram-negative bacteria worldwide has greatly compromised the efficacy of carbapenem antibiotics, which has gotten renewed attention to the importance of polymyxin antibiotics for multidrug-resistant (MDR) infections. Recently, Sophia David and colleagues ${ }^{2}$ reported the epidemic of carbapenem-resistant Klebsiella pneumoniae (CRKP) in Europe, which raised concern that mobile carbapenemase resistance determinants were widely spread in European hospital settings, and inter-hospital spread is far more frequent within, rather than between, countries. However, limited information regarding the co-occurrence of carbapenemases and $\mathrm{mcr}$ genes in the Klebsiella pneumoniae (K. pneumoniae) isolates have been provided. Plasmid-mediated resistance genes $m c r$ as well as tet(X3/X4) have been widespread in bacterial species of animal, human, and environment origin as well as human and animal gut microbiomes worldwide, where is a huge ARG reservoir with a high horizontal gene transfer possibility. ${ }^{1,3-5}$ Several studies $^{6-8}$ illustrated that the newly described mcr-9 has spread beyond the United States into Europe and Asia, and into other Enterobacteriaceae species. Of clinical concern is the inevitable spread of a plasmid harboring the mcr-9 gene into a CRKP isolate, which has been listed by the World Health Organization as a critical priority antibiotic-resistant bacterial pathogen for which new antibiotics are urgently needed. ${ }^{9}$ In response to this potential clinical problem, we download > 1717 K. pneumoniae genomes isolated from 244 hospitals in 32 European countries ${ }^{2}$ and the 14,764 complete bacterial genome sequence (accessed 26 July 2019), and explored the distribution of plasmid-mediated resistance genes $\mathrm{mcr}$ and tet(X3/X4).

We are surprised to find that the complete mcr-9 gene (nucleotide $=100 \%$ ) was present in $28 \mathrm{~K}$. pneumoniae isolates of 1717 K. pneumoniae strains from Belgium $(n=2)$, Denmark $(n=1)$, Montenegro $(n=13)$, Poland $(n=1)$, Romania $(n=2)$, Serbia $(n=1)$, Slovenia $(n=1)$, and Spain $(n=7)$ (Table S1). Additionally, 23 of the $28 \mathrm{~K}$. pneumoniae isolates of human origin were CRKP strains and only five were carbapenem-susceptive isolates (Table S1). As reported, these mcr-9-harbouring strains were isolated from patients in Europe between 2013 and 2014. These results suggest that $m c r-9$ gene might have been presented in Europe for a long time and already spread to the CRKP isolates, which is a major cause of both hospital- and community-acquired infections. ${ }^{2}$

To further analyze these mor-9-positive $K$. pneumoniae isolates, the resistome of the draft genome was analyzed using the Comprehensive Antibiotic Resistance Database. ${ }^{10}$ Interestingly, the mcr-9 gene was co-existed with various carbapenemase-encoding genes: in eleven isolates with NDM-1, eight with VIM-1, and two with OXA-48 (Fig. 1(A)). It should be noted that the mcr-9- and NDM-1-carrying isolates were distributed in Denmark $(n=1)$, Montenegro $(n=8)$, Romania $(n=1)$, and Serbia $(n=1)$, as well as several other beta-lactam resistance determinants (for example, TEM-1, CMY-16, OXA-10 and SHV-11) (Fig. 1(A) and Table S1). Moreover, the mcr-9- and VIM-1-harbouring isolates were dominant in Spain $(n=7)$ and Slovenia $(n=1)$, as well as two beta-lactamase-encoding genes (non-carbapenemases) (CTX-M-9 and SHV-11) (Fig. 1(A) and Table S1). It is worrying that a CRKP isolate from Spain in 2014 was carrying $m c r-9$, VIM-1, and OXA-48 genes simultaneously. The presence of $m c r-9$ in CRKP isolates from patients is of critical importance as $m c r-9$ could be present in hospital-borne outbreaks CRE strains in the future.

From whole-genome shotgun (WGS) data of the $28 \mathrm{mcr}$ 9-positive $K$. pneumoniae isolates, sequences types (STs) were extracted and assigned to nine different types, i.e., 274, 461, 15, 16, 416, 1890, 37, 1942, and 147 (Fig. 1(A)). Phylogenetic analysis 


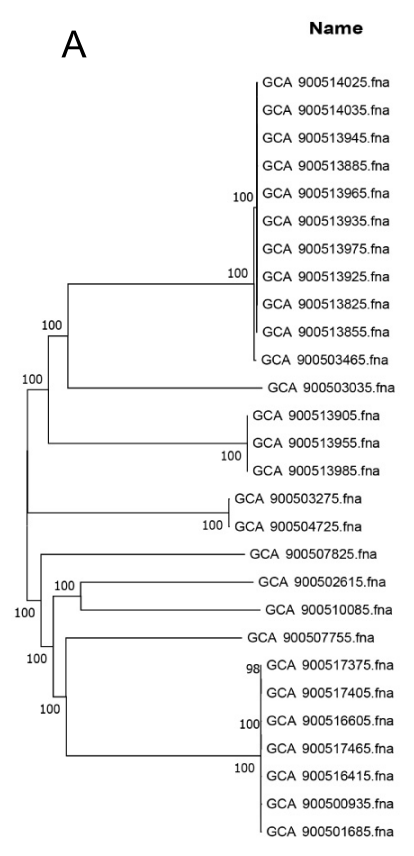

\begin{tabular}{|c|c|c|c|c|c|}
\hline ST & mcr-9 & NDM-1 & VIM-1 & OXA-48 & $\begin{array}{l}\text { Geographical } \\
\text { distribution }\end{array}$ \\
\hline 274 & + & - & - & - & Montenegro \\
\hline 274 & + & + & - & - & Montenegro \\
\hline 274 & + & + & - & - & Montenegro \\
\hline 274 & + & + & - & - & Montenegro \\
\hline 274 & + & - & - & - & Montenegro \\
\hline 274 & + & + & - & - & Montenegro \\
\hline 274 & + & + & - & - & Montenegro \\
\hline 274 & + & + & - & - & Montenegro \\
\hline 274 & + & + & - & - & Montenegro \\
\hline 274 & + & + & - & - & Montenegro \\
\hline 274 & + & + & - & - & Serbia \\
\hline 461 & + & - & + & - & Slovenia \\
\hline 15 & + & - & - & - & Montenegro \\
\hline 15 & + & - & - & - & Montenegro \\
\hline 15 & + & - & - & - & Montenegro \\
\hline 16 & + & + & - & - & Romania \\
\hline 16 & + & - & - & - & Romania \\
\hline 416 & + & - & - & + & Belgium \\
\hline 1890 & + & + & - & - & Denmark \\
\hline 37 & + & - & - & - & Poland \\
\hline 1942 & + & - & - & - & Belgium \\
\hline 147 & + & - & + & - & Spain \\
\hline 147 & + & - & + & - & Spain \\
\hline 147 & + & - & + & - & Spain \\
\hline 147 & + & - & + & - & Spain \\
\hline 147 & + & - & + & - & Spain \\
\hline 147 & + & - & + & + & Spain \\
\hline 147 & + & - & + & - & Spain \\
\hline
\end{tabular}

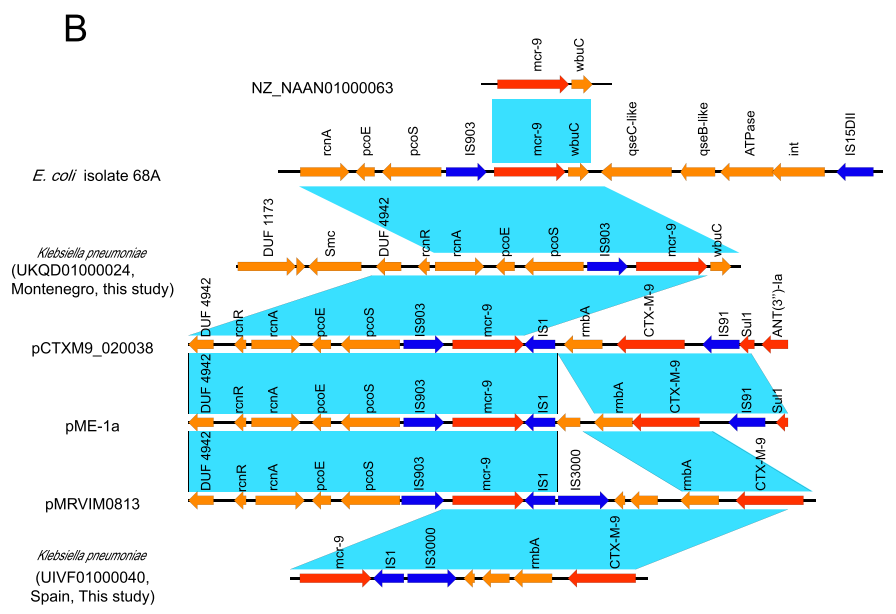

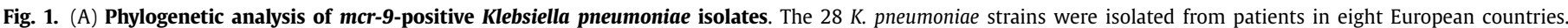

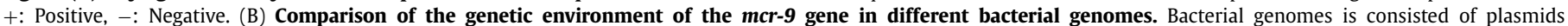

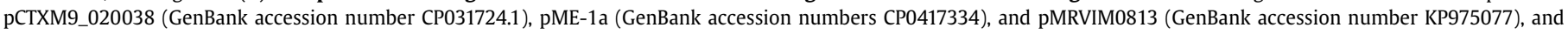

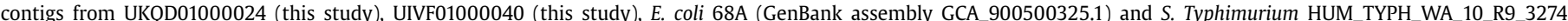

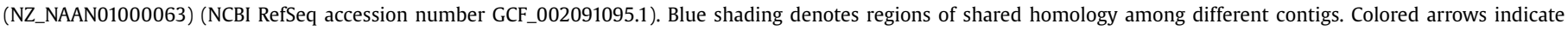
open reading frames, with orange, dark blue, and red arrows representing others, mobile elements, and antibiotic resistance genes, respectively.

suggested that mcr-9-carrying K. pneumoniae isolates show a highly geographical clustering pattern ${ }^{2}$ (Fig. $1(\mathrm{~A})$ ). Isolates from patients in the same hospital were clustered into one clade, for example, in Spain and Montenegro. Overall, the K. pneumoniae isolates from different countries were genetically diverse, suggesting that the mcr-9-positive $K$. pneumoniae isolates were also genetically diverse and that mor-9 could disseminate among different $K$. pneumoniae isolates, mainly by nosocomial transmission. ${ }^{2}$ Nowadays, all known mor genes have been detected in various Gram-negative bacterial species, whereas a small number of studies have shown the presence of $m c r-1, m c r-3, m c r-7$, and $m c r-8$ in $K$. pneumoniae isolates from animal and human origin at relatively low detection rate. ${ }^{11-14}$ The presence of $m c r-9$ in the CRKP isolates indicated that this novel $m c r$ gene may already be widely spread among $K$. pneumoniae isolates of human origin in Europe.

We subsequently searched $m c r-9$ gene in 14,764 complete bacterial genome sequence and NCBI-nr database (14 October 2019) in the NCBI, to fully understand the prevalence of $m c r-9$ gene in Klebsiella species isolates. Interestingly, the mcr-9 gene (identity $>99 \%$ and $100 \%$ query coverage) was present in various bacterial genomes, including three Klebsiella species isolates consisting of K. pneumoniae $(n=4), K$. quasipneumoniae $(n=1)$, and $K$. oxytoca $(n=3)$ (Table S3). Therefore, further studies focusing on the epidemiology and transmission mechanism of $\mathrm{mor}$ genes, in particular mcr-9 in Klebsiella species of human origin are warranted to better understand the public health threat of emergence of antibiotic resistance among clinical K. pneumoniae.

Contigs carrying mcr-9 in $28 \mathrm{~K}$. pneumoniae isolates could be classified into two groups (for example, GCA_900513855.1 and GCA_900501685.1) (Table S2). Genetic environments analysis indicated that the presence of insertion element IS903 and wbuC (a cupin fold metalloprotein), in the mcr-9 (GCA_900513855.1, $\sim 28 \mathrm{~kb})$ upstream and downstream flanking, respectively, similar to (identity >99\%) the plasmid sequences of pME-1a,
pCTXM9_020038, and pMRVIM0813, and contigs from of E. coli isolate 68A and NZ_NAAN01000063 from Salmonella ${ }^{15}$ (Fig. 1(B)). Additionally, mcr-9 in another contig $\sim 8.7 \mathrm{~kb}$ was in the upstream of two insertion element IS1 and IS3000, as well as a beta-lactamase-encoding resistance gene CTX-M-9, which similar to the plasmid sequence of pMRVIM0813. We did not detect the downstream regulatory genes (qseC and qseB) found in the isolates that harbor mcr-9.8,16 Moreover, we were unable to determine whether a complete IS903 element is upstream due to a short mcr-9-bearing contig that is available for comparison (Fig. 1(B)). Therefore, a long-read sequencing coupled with a hybrid assembly method is needed to fully evaluate and monitor the transfer and development of ARGs, especially mcr-9 among CRKP isolates.

Although two unique plasmid-mediated tigecycline resistance genes firstly discovered in bacteria of animal origin in China and subsequently identified in many bacterial isolates of human, animal and environment origin, including Klebsiella species, as well as human and animal gut microbiomes, ${ }^{1,4,5}$ none of them was detected in the $1717 \mathrm{~K}$. pneumoniae strains in Europe.

In summary, we reported the discovery of mcr-9 gene in 28 clinical K. pneumoniae strains of human origin in eight European countries. Importantly, the mcr-9 gene was co-existed with different carbapenemase-encoding genes in the same strains. The spread of $m c r-9$, NDM-1, VIM-1, and OXA-48 and other beta-lactam resistance determinants (non-carbapenemase) carrying by CRKP appears likely to be by plasmid dissemination, as the genes identified in isolates belonging to a diverse set of STs distributed in different hospitals in Europe. It is noteworthy that all these mcr-9-positive CRKP strains were isolated between 2013 and 2014, highlighting an earlier presence of mcr-9 among CRKP around the world than previously known. These findings raise the likelihood of ongoing undetected mcr-9 gene spread among CRE strains. Therefore, further study is urgently needed to understand the prevalence and dissemination of mcr-9, especially in CRE and 
CRKP strains, and effective measures should be taken to control its spread.

\section{Author contributions}

G.F.G. designed the study. Y.N.W. and F.L. collected and downloaded the datasets. Y.N.W., F.L., Y.F.H., B.L.Z., G.P.Z., and G.F.G. analyzed and interpreted the data. Y.N.W. and G.F.G. wrote the draft of the manuscript. All authors discussed, reviewed and approved the final report.

\section{Supplementary Tables}

Supplementary information is available for this paper. Correspondence and requests for materials should be addressed to G.F.G.

\section{Declaration of Competing Interest}

The authors declare no competing interests.

\section{Acknowledgements}

This work was supported by the Strategic Priority Research Program of the Chinese Academy of Sciences (XDB29010000), the External Cooperation Program of CAS (153211KYSB20160001), and National Key R\&D Program of China (2018YFC1603803).

\section{Supplementary materials}

Supplementary material associated with this article can be found, in the online version, at doi:10.1016/j.jinf.2019.12.016.

\section{References}

1. Wang Y., Liu F., Zhu B., Gao G.F.. Metagenomic data screening reveals the distribution of mobilized resistance genes tet(X), mor and carbapenemase in animals and humans. I Infect 2019. doi:10.1016/j.jinf.2019.09.003.

2. David S., Reuter S., Harris S.R., Glasner C., Feltwell T., Argimon S., et al. Epidemic of carbapenem-resistant Klebsiella pneumoniae in Europe is driven by nosocomial spread. Nat Microbiol 2019;4(11):1919-29.

3. Wang Y., Hu Y., Cao J., Bi Y., Lv N., Liu F., et al. Antibiotic resistance gene reservoir in live poultry markets. J Infect 2019;78(6):445-53.

4. He T., Wang R., Liu D., Walsh T.R., Zhang R., Lv Y., et al. Emergence of plasmidmediated high-level tigecycline resistance genes in animals and humans. Nat Microbiol 2019;4(9):1450-6.

5. Sun J., Chen C., Cui C.Y., Zhang Y., Liu X., Cui Z.H., et al. Plasmid-encoded tet(X) genes that confer high-level tigecycline resistance in Escherichia coli. Nat Microbiol 2019;4(9):1457-64.

6. Chavda K.D., Westblade L.F., Satlin M.J., Hemmert A.C., Castanheira M., Jenkins S.G., et al. First report of blaVIM-4- and mcr-9-Coharboring Enterobacter species isolated from a pediatric patient. mSphere 2019;4(5) e00629-19.

7. Borjesson S., Greko C., Myrenås M., Landén A, Nilsson O., Pedersen K.. A link between the newly described colistin resistance gene mcr-9 and clinical Enterobacteriaceae isolates carrying blaSHV-12 from horses in Sweden. J Global Antimicrob Resist 2019 S2213-7165(19)30205-X. doi:10.1016/j.jgar.2019.08.007.

8. Kieffer N., Royer G., Decousser J.W., Bourrel A.S., Palmieri M., Ortiz De La Rosa J.M., et al. mcr-9, an inducible gene encoding an acquired phosphoethanolamine transferase in Escherichia coli, and its origin. Antimicrob Agents Chemother 2019;63(9) e00965-19.

9. Tacconelli E., Carrara E., Savoldi A., Harbarth S., Mendelson M., Monnet D.L., et al. Discovery, research, and development of new antibiotics: the WHO priority list of antibiotic-resistant bacteria and tuberculosis. Lancet Infect Dis 2018;18(3):318-27.

10. Jia B., Raphenya A.R., Alcock B., Waglechner N., Guo P., Tsang K.K., et al. CARD 2017: expansion and model-centric curation of the comprehensive antibiotic resistance database. Nucleic Acids Res 2017;45(D1):D566-73.

11. Liu Y.Y., Wang Y., Walsh T.R., Yi L.X., Zhang R., Spencer J., et al. Emergence of plasmid-mediated colistin resistance mechanism MCR-1 in animals and human beings in China: a microbiological and molecular biological study. Lancet Infect Dis 2016;16(2):161-8.

12. Yang Y.Q., Li Y.X., Lei C., Zhang A.Y., Wang H.N.. Novel plasmid-mediated colistin resistance gene mcr-7.1 in Klebsiella pneumoniae. J Antimicrob Chemothery 2018;73(7):1791-5.

13. Wang X., Wang Y., Zhou Y., Li J., Yin W, Wang S., et al. Emergence of a novel mobile colistin resistance gene, $m c r-8$, in NDM-producing Klebsiella pneumoniae. Emerg Microbes Infect 2018;7(1):122.
14. Yin W., Li H., Shen Y., Liu Z., Wang S., Shen Z., et al. Novel plasmid-mediated colistin resistance gene mcr-3 in Escherichia coli. mBio 2017;8(3) e00543-00517.

15. Carroll L.M., Gaballa A., Guldimann C., Sullivan G., Henderson L.O., Wiedmann M.. Identification of novel mobilized colistin resistance gene mcr-9 in a multidrug-resistant, colistin-susceptible Salmonella enterica serotype Typhimurium isolate. mBio 2019;10(3) e00853-19.

16. Yuan Y., Li Y., Wang G., Li C., Xiang L., She J., et al. Coproduction of MCR-9 and NDM-1 by colistin-resistant enterobacter hormaechei isolated from bloodstream infection. Infect Drug Resist 2019;12:2979-85.

Yanan Wang ${ }^{1}$

College of Animal Science and Veterinary Medicine, Henan Agricultural University, Zhengzhou, Henan 450046, China CAS Key Laboratory of Pathogenic Microbiology and Immunology, Institute of Microbiology, Chinese Academy of Sciences, Beijing 100101, China

Fei Liu ${ }^{1}$

CAS Key Laboratory of Pathogenic Microbiology and Immunology, Institute of Microbiology, Chinese Academy of Sciences, Beijing 100101, China

Yongfei Hu College of Animal Science and Technology, China Agricultural University, Beijing 100193, China

Gaiping Zhang

College of Animal Science and Veterinary Medicine, Henan Agricultural University, Zhengzhou, Henan 450046, China

Baoli Zhu

CAS Key Laboratory of Pathogenic Microbiology and Immunology, Institute of Microbiology, Chinese Academy of Sciences, Beijing 100101, China

George Fu Gao*

CAS Key Laboratory of Pathogenic Microbiology and Immunology, Institute of Microbiology, Chinese Academy of Sciences, Beijing 100101, China

Chinese Center for Disease Control and Prevention (China CDC), Beijing 102206, China

*Corresponding author. E-mail address: gaof@im.ac.cn (G.F. Gao)

1 These authors contributed equally to this work. Accepted 23 December 2019 Available online 15 January 2020

https://doi.org/10.1016/j.jinf.2019.12.016

(C) 2020 The British Infection Association. Published by Elsevier Ltd. All rights reserved.

\section{Management and control of an outbreak of vaccine-preventable severe pneumococcal disease at a shipyard in Norway}

\section{Dear Editor,}

The interesting systematic review by Amin-Chowdhury and colleagues provides information about outbreaks of severe pneumococcal disease (SPD) in closed settings that occurred in the conjugate vaccines era ${ }^{1}$. It shows that vaccine-type SPD outbreaks are still occurring and it highlights the lack of consensus on how to manage such outbreaks. In the following, we will describe how we managed a recent outbreak of SPD in Norway.

In March 2019, Møre and Romsdal Hospital Trust notified the Norwegian Institute of Public Health (NIPH) about a cluster of 


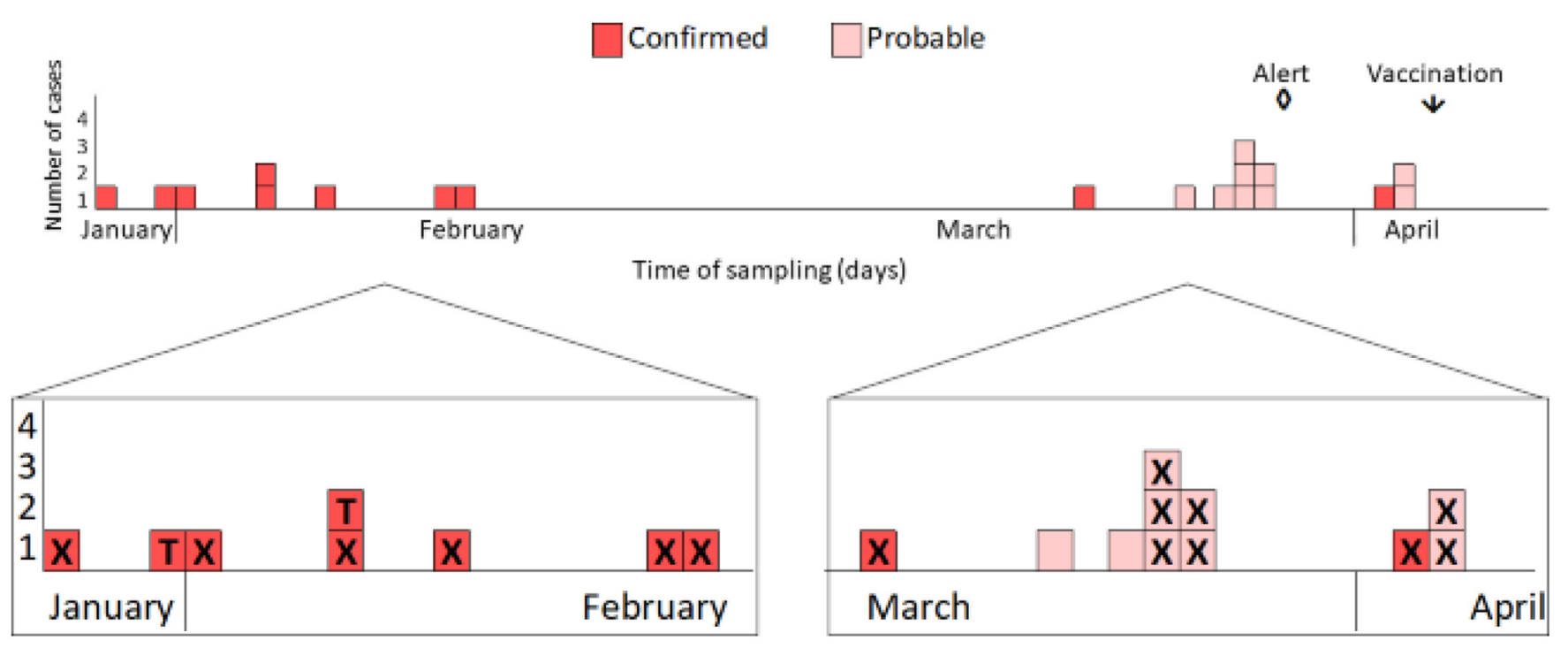

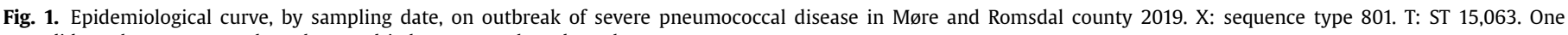
case did not have any samples taken, and is hence not plotted on the curve.

SPD amongst men working in shipyards in Møre and Romsdal county. Serotype data from NIPH were available for nine of the cases - all were serotype 4 . The majority of cases had been working at one specific shipyard. Municipal medical officers (MMO), the Norwegian Labour Inspection Authority (NLIA), and NIPH formed a multidisciplinary outbreak team to investigate and control the outbreak.

We formed specific case definitions: each case had to have resided in Møre and Romsdal county in the period from 1. January 2019 onwards AND: Confirmed: had invasive pneumococcal disease (IPD) with serotype 4 isolated from a normally sterile site. Probable: worked at the specific shipyard AND had a clinical presentation compatible with lower respiratory tract infection or IPD, but without microbiological confirmation OR serotype 4 isolated from a non-sterile medium (e.g. nasopharyngeal swab or sputum culture).

We identified 20 cases, ten confirmed and ten probable in the period between 28. January and 3. April (Fig. 1). All available isolates were serotype 4 (10 confirmed, 7 probable) and were susceptible to penicillin. Fifteen isolates were sequence type (ST) 801, while two were a single locus variant of 801, ST 15,063.

All cases were men between 20 and 60 years, with a mean age of 47 years. Fifteen were hospitalized. Four were Norwegian citizens, the remaining came from other European countries. Seven cases smoked. One case had an underlying medical risk condition. Immunization history against pneumococci were unknown for all. The cases had several professions; mostly related to interior outfitting and metal welding. Approximately 1800 individuals worked at the shipyard in the time period. Many of them lived in temporary accommodation. At an on-site inspection of the shipyard, NLIA observed a polluted atmospheric work environment and little use of personal protective equipment.

Several measures were put in place to control the outbreak, including information and advice to raise symptom awareness and to reinforce hand and respiratory hygiene, vaccination and occupational corrections. Local medical clinics and hospital were alerted about the outbreak and advised to have a low threshold to admit and treat suspected cases. MMO held information meetings with shift leaders, and written information about SPD in several languages was distributed to workers to increase SPD awareness. Intensified hygiene measures were implemented at the ship yard and housing quarters.
NLIA ordered immediate occupational corrections related to controlling the atmospheric work environment. NIPH recommended vaccination with the 13 -valent conjugate vaccine (PCV13) to interrupt transmission and prevent disease. Both the PCV13 and the 23-valent polysaccharide vaccine provide protection against serotype 4, but PCV13 was preferred as this may also affect colonization. As several work tasks were conducted in parallel process in confined spaces with suboptimal ventilation, we were unable to identify a single target group for vaccination. Hence, the shipyard offered vaccination to all workers. Occupational health service promptly vaccinated all workers during a four-day period.

Contrary to the majority of studies included in the systematic review, NIPH did not recommend chemoprophylaxis. As the workers were otherwise healthy (i.e. no high risk group like old age, immunocompromising conditions etc.), and since it was impossible to target a specific group of workers, NIPH deemed it undesirable to distribute antibiotics to 1800 asymptomatic workers, with the possibility of inducing antimicrobial resistance and possible side effects.

Due to high turnover of personnel it was not possible to calculate an attack rate. We did not find any new cases after control measures were implemented. No deaths have been reported in relation to the outbreak.

This outbreak closely resembles one of the outbreaks described in the systematic review; between April and June 2015, an outbreak with serotype 4, ST 801 occurred at a shipyard in Belfast ${ }^{2}$. We are also aware of an outbreak this fall, 2019, at a shipyard in Finland with serotype 4 (ST 801), 8 and $12 \mathrm{~F}^{3}$. Although welders are a known risk group for SPD, ${ }^{4}$ in all these three outbreaks, people who worked closely alongside welders were also infected. In addition to exposure to welding fumes, the crammed and poorly ventilated working conditions, and possibly housing conditions, may have increased the risk of developing SPD and facilitated the transmission of pneumococci in this closed setting. Overall, this Norwegian outbreak extends the knowledge about how to manage and control outbreaks of SPD in closed settings.

\section{Declaration of Competing Interest}

None. 


\section{References}

1. Amin-Chowdhury Z., Iyanger N., Ramsay M.E., Ladhani S.N. Outbreaks of severe pneumococcal disease in closed settings in the conjugate vaccines era, 20102018: a systematic review to inform national guidance in the UK. J Infect 2019. doi:10.1016/j.jinf.2019.10.009.

2. Ewing J., Patterson L., Irvine N., Doherty L., Loughrey A., Kidney J., et al. Serious pneumococcal disease outbreak in men exposed to metal fume - detection, response and future prevention through pneumococcal vaccination. Vaccine 2017;35(32):3945-50. doi:10.1016/j.vaccine.2017.06.010.

3. Linkevicius M., Cristea V., Siira L., Mäkelä H., Toropainen M., Pitkäpaasi M., et al. Outbreak of invasive pneumococcal disease among shipyard workers, Turku, Finland, May to November 2019. Euro Surveill 2019;24(49):1900681. doi:10.2807/1560-7917.ES.2019.24.49.1900681.

4. Wong A., Marrie T.J., Garg S., Kellner J.D., Tyrrell G.J., Group S. Welders are at increased risk for invasive pneumococcal disease. Int J Infect Dis 2010;14(9):e7969. doi:10.1016/j.ijid.2010.02.2268.

Jacob Dag Berild, Anneke Steens, Brita Askeland Winje Department of Vaccine Preventable Diseases, Division of Infection Control and Environmental Health, Norwegian Institute of Public Health, Postboks 222 Skøyen, Oslo 0213, Norway

Tor Erik Danielsen Norwegian Labour Inspection Authority, Postboks 4720 Torgarden, Trondheim 7468, Norway

Jan Håkon Fjeldheim Vestnes Municipality, Brugata 10, Vestnes 6390, Norway

Helene Daae-Qvale Holmemo Department of medicine, Ålesund hospital, Møre and Romsdal Hospital Trust, Postboks 1600, Ålesund 6026, Norway

Didrik Frimann Vestrheim Department of Vaccine Preventable Diseases, Division of Infection Control and Environmental Health, Norwegian Institute of Public Health, Postboks 222 Skøyen, Oslo 0213, Norway

*Corresponding author.

E-mail addresses: jacobdag.berild@fhi.no (J.D. Berild), anneke.steens@fhi.no (A. Steens), brita.askeland.winje@fhi.no (B.A. Winje), tor.erik.danielsen@arbeidstilsynet.no (T.E. Danielsen), jan.fjeldheim@vestnes.kommune.no (J.H. Fjeldheim), helene.daae-qvale.holmemo@helse-mr.no (H.D.-Q. Holmemo), didrikfrimann.vestrheim@fhi.no (D.F. Vestrheim)

Accepted 23 December 2019 Available online 15 January 2020

https://doi.org/10.1016/j.jinf.2019.12.015

(C) 2020 The British Infection Association. Published by Elsevier Ltd. All rights reserved.

\section{Leishmania infection in psoriasis}

Dear Editor,

In this Journal Brunet and colleagues ${ }^{1}$ discussed reactivation of latent infections in the context of chronic disease, solid organ transplantation or long-term immunosuppressive treatment. We recently observed the reactivation of Leishmania infection in a 46-year-old patient receiving methotrexate for psoriasis, who was diagnosed with visceral leishmaniasis (VL) showing a mucocutaneous involvement. We analyzed the epidemiologic and clinical characteristics of all cases of leishmaniasis in patients with psoriasis found through a review of the literature.

Our patient was admitted into the Infectious Disease Unit of Paolo Giaccone hospital, in Palermo, with a painless and ulcerated
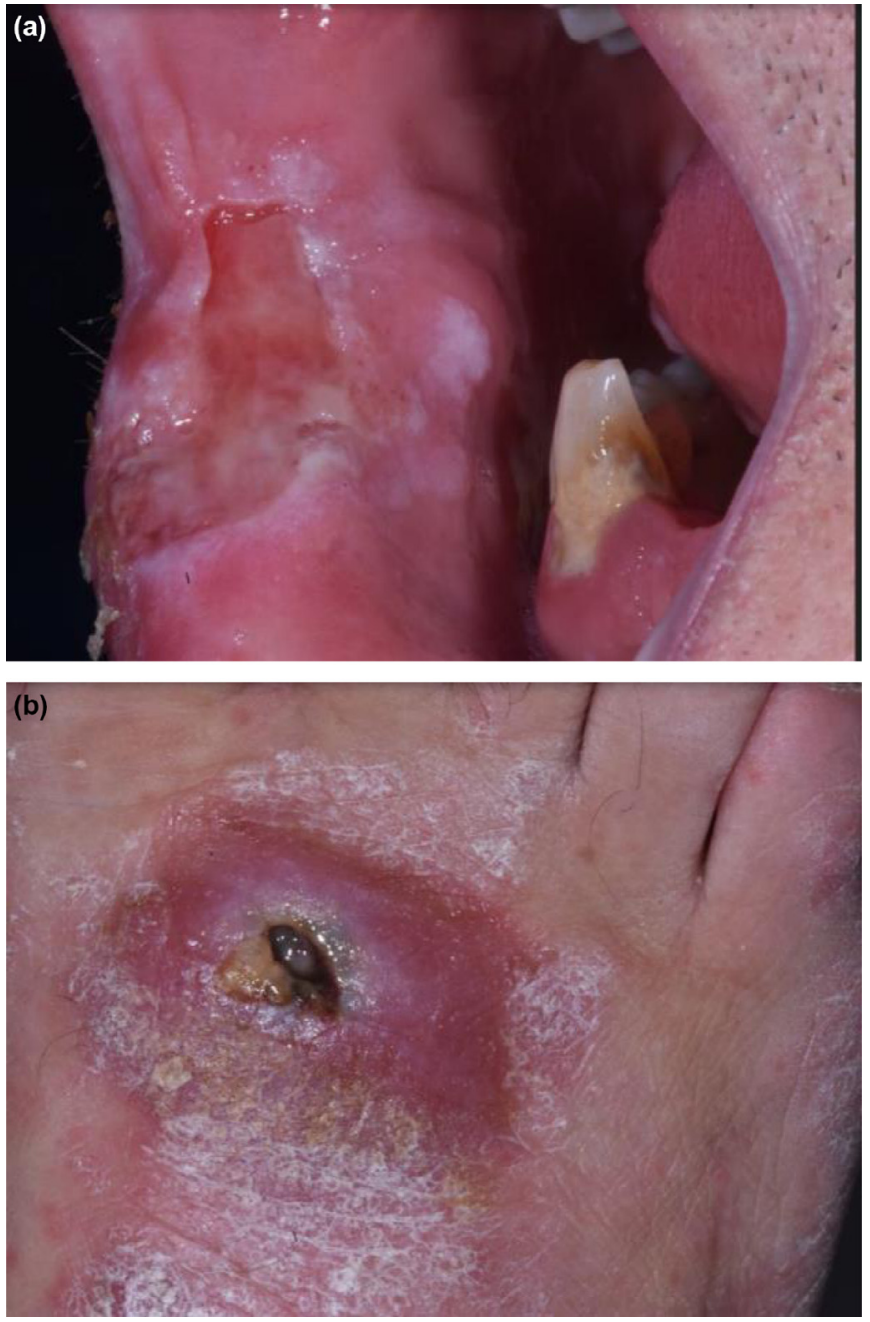

Figs. 1a and b. Ulcerated lesions by Leishmania onto the oral mucosa (a) and right foot (b) in a 46-year-old patient with psoriasis.

lesion onto the oral mucosa (Fig. 1a), two nodular ulcerated lesions on the right knee and another one on instep of the right foot appeared one month before (Fig. $1 \mathrm{~b}$ ). The patient did not travel outside Italy during the last year. He had been suffering from lowgrade fever in the last month.

Considering the above findings leishmaniasis was suspected and a needle aspiration of oral and cutaneous lesions was arranged in order to perform microscopy and Leishmania-PCR, which were positive for Leishmania. Laboratory tests exhibited: WBC 4970/mmc, Hb 13.9g/dl, C Reactive Protein, $26.9 \mathrm{mg} / \mathrm{L}$; positive serology for Leishmania (IgG 1/3200) and positive LeishmaniaPCR test on peripheral blood. Abdominal US examination revealed splenomegaly $(14 \mathrm{~cm})$; methotrexate was suspended and liposomal Amphotericin B, $4 \mathrm{mg} / \mathrm{kg}$ per day for 10 days, followed by two further administrations two weeks later was started. Cutaneous and mucosal lesions improved at the end of the first 10 days of therapy and completely vanished after two further administrations, 40 days from the beginning of treatment. Leishmania-PCR on peripheral blood after 10 days of therapy was negative.

Table 1 shows the literature data about characteristics, therapy and outcome of patients with psoriasis and leishmaniasis.

Leishmaniasis is a vector-born chronic infectious disease caused by protozoa of the genus Leishmania and transmitted to humans by the bite of phlebotomine sandflies. In Europe, the Mediterranean countries are the most affected areas. Leishmania parasite 
Table 1

Detailed characteristics of patients with psoriasis or psoriatic arthritis in whom leishmaniasis developed.

\begin{tabular}{|c|c|c|c|c|c|c|c|c|c|c|c|c|}
\hline $\begin{array}{l}\text { First author Year } \\
\text { Number of patients }\end{array}$ & $\begin{array}{l}\text { Risk factors for } \\
\text { Leishmania } \\
\text { infection }\end{array}$ & Age, y/sex & Disease & $\begin{array}{l}\text { Immunosuppressive } \\
\text { treatment }\end{array}$ & $\begin{array}{l}\text { Duration } \\
\text { months }\end{array}$ & $\begin{array}{l}\text { Form of } \\
\text { Leishmania } \\
\text { Infection }\end{array}$ & PCR & Microscopy. & Serology & Treatment & $\begin{array}{l}\text { Suspension } \\
\text { of immuno- } \\
\text { suppressive } \\
\text { therapy }\end{array}$ & Outcome \\
\hline $\begin{array}{l}\text { Romani-Costa et al., 2004, } \\
\text { Enferm Infecc Microbiol } \\
\text { Clin }\end{array}$ & $\begin{array}{l}\text { Endemic area } \\
\text { Spain }\end{array}$ & $55 / \mathrm{M}$ & PSA & Infliximab & 9 & $\mathrm{VL}$ & & & & Meglumine antimoniate & & Cured \\
\hline $\begin{array}{l}\text { Saha et al., 2006, Br J } \\
\text { Dermatol }\end{array}$ & $\begin{array}{l}\text { Traveler returning } \\
\text { from Mexico, Spain, } \\
\text { Greece, Malta and } \\
\text { Turkey }\end{array}$ & $37 / \mathrm{M}$ & PSA & $\begin{array}{l}\text { Metotrexate } \\
\text { Sulfasalazine }\end{array}$ & 36 & $\mathrm{CL}$ & Pos SB & Pos SB & Pos & $\begin{array}{l}\text { Iv sodium } \\
\text { stibogluconate }\end{array}$ & $\mathrm{NR}$ & Cured \\
\hline $\begin{array}{l}\text { Balato et al., 2008, } \\
\text { Dermatology }\end{array}$ & $\begin{array}{l}\text { Endemic area } \\
\text { Italy }\end{array}$ & $42 / \mathrm{M}$ & $\mathrm{P}$ & $\begin{array}{l}\text { Efalizumab } \\
\text { cyclosporina }\end{array}$ & 24 & $\mathrm{VL}$ & $\begin{array}{l}\text { Pos BM } \\
\text { Pos } \\
\text { PB }\end{array}$ & Pos BM & Pos & None & Yes & Cured \\
\hline $\begin{array}{l}\text { Tektonidou and Skopouli, } \\
\text { 2008, Clin Rheumatol }\end{array}$ & $\begin{array}{l}\text { Endemic area } \\
\text { Greece }\end{array}$ & $45 / \mathrm{M}$ & PSA & $\begin{array}{l}\text { Methotrexate } \\
\text { Infliximab Prednisolone }\end{array}$ & 60 & $\mathrm{VL}$ & $\begin{array}{l}\text { Pos } \\
\text { BM }\end{array}$ & $\begin{array}{l}\mathrm{Neg} \\
\mathrm{BM}\end{array}$ & Neg & $\begin{array}{l}\text { Lyophilized } \\
\text { amphotericin }\end{array}$ & Yes & Cured \\
\hline $\begin{array}{l}\text { De Leonardis et al., 2009, } \\
\text { Clin Exp Rheumatol }\end{array}$ & $\begin{array}{l}\text { Endemic area } \\
\text { Italy }\end{array}$ & $63 / \mathrm{M}$ & PSA & $\begin{array}{l}\text { Methotrexate } \\
\text { Infliximab } \\
\text { azathioprine prednisolone }\end{array}$ & 36 & $\mathrm{VL}$ & ND & PosBM & Neg & $\begin{array}{l}\text { Liposomal amphotericin } \\
\text { B }\end{array}$ & Yes & Cured \\
\hline $\begin{array}{l}\text { Khan et al., 2010, BMJ } \\
\text { case rep }\end{array}$ & $\begin{array}{l}\text { Traveler returning } \\
\text { from Malta }\end{array}$ & $74 / \mathrm{F}$ & PSA & $\begin{array}{l}\text { Adalimumab } \\
\text { methotrexate }\end{array}$ & 21 & $\begin{array}{l}\mathrm{CL} \\
\mathrm{VL}\end{array}$ & $\begin{array}{l}\text { Pos BM } \\
\text { Pos SB }\end{array}$ & $\begin{array}{l}\text { pos BM } \\
\text { pos SB }\end{array}$ & pos & $\begin{array}{l}\text { Intralesional } \\
\text { meglumine antimoniate } \\
\text { and liposomal } \\
\text { Amphotericin }\end{array}$ & Yes & Cured \\
\hline $\begin{array}{l}\text { Romero-Mate et al., 2012, } \\
\text { Dermatol Online J }\end{array}$ & $\begin{array}{l}\text { Endemic area } \\
\text { Spain }\end{array}$ & $59 / \mathrm{F}$ & PSA & $\begin{array}{l}\text { Adalimumab } \\
\text { methotrexate }\end{array}$ & 84 & CL & Pos & neg & ND & $\begin{array}{l}\text { Intralesional } \\
\text { meglumine antimoniate }\end{array}$ & No & Cured \\
\hline $\begin{array}{l}\text { Besada et al., 2013, } \\
\text { Rheumatol Int }\end{array}$ & $\begin{array}{l}\text { Traveler returning } \\
\text { from } \\
\text { southern Spain }\end{array}$ & $72 / \mathrm{F}$ & PSA & Methotrexate Etanercept & 288 & $\mathrm{VL}$ & Pos BM & Pos BM & ND & $\begin{array}{l}\text { Liposomal amphotericin } \\
\text { B }\end{array}$ & Yes & Cured \\
\hline $\begin{array}{l}\text { Hernandez-Torres et al., } \\
\text { 2013, Scand J Infect Dis }\end{array}$ & $\begin{array}{l}\text { Endemic area } \\
\text { Spain }\end{array}$ & $50 / \mathrm{M}$ & $\mathrm{P}$ & $\begin{array}{l}\text { Corticosteroids } \\
\text { infliximab }\end{array}$ & 36 & $\mathrm{CL}$ & Pos SB & Pos SB & Pos & $\begin{array}{l}\text { Liposomal amphotericin } \\
\text { B }\end{array}$ & Yes & Cured \\
\hline $\begin{array}{l}\text { Guedes-Barbosa et al., } \\
\text { 2013, Semin Arthritis } \\
\text { Rheum (two patients) }\end{array}$ & $\begin{array}{l}\text { Endemic area } \\
\text { Brazil }\end{array}$ & $\begin{array}{l}39 / \mathrm{M} \\
50 / \mathrm{M}\end{array}$ & PSA (2) & $\begin{array}{l}\text { Prednisone } \\
\text { methotrexate adalimumab } \\
\text { (2) } \\
\text { Infliximab (1) }\end{array}$ & $\begin{array}{l}12 \\
14\end{array}$ & $\begin{array}{l}\mathrm{MCL} \\
\mathrm{CL}\end{array}$ & $\begin{array}{l}\text { ND } \\
\text { ND }\end{array}$ & $\begin{array}{l}\text { Pos SB } \\
\text { Pos SB }\end{array}$ & NDND & $\begin{array}{l}\text { Meglumine antimoniate } \\
\text { (2) }\end{array}$ & $\begin{array}{l}\text { No } \\
\text { Yes }\end{array}$ & $\begin{array}{l}\text { Cured } \\
\text { Cured }\end{array}$ \\
\hline $\begin{array}{l}\text { Hadjipetrou et al., 2014, } \\
\text { Infez Med }\end{array}$ & $\begin{array}{l}\text { Endemic area } \\
\text { Greece }\end{array}$ & $65 / \mathrm{M}$ & PSA & Methotrexate & 24 & $\mathrm{VL}$ & Pos BM & Pos BM & Pos & $\begin{array}{l}\text { Liposomal amphotericin } \\
\text { B }\end{array}$ & Yes & Cured \\
\hline $\begin{array}{l}\text { Catala et al., 2014, Actas } \\
\text { Dermosifiliogr }\end{array}$ & $\begin{array}{l}\text { Endemic area } \\
\text { Spain }\end{array}$ & $33 / \mathrm{M}$ & PSA & Infliximab & 24 & $\mathrm{CL}$ & ND & $\begin{array}{l}\text { Pos SB } \\
\text { Neg BM }\end{array}$ & Pos & $\begin{array}{l}\text { Intralesional } \\
\text { meglumine antimoniate }\end{array}$ & Yes & Cured \\
\hline $\begin{array}{l}\text { Rakotonarivo et al., 2018, } \\
\text { JAAD case rep }\end{array}$ & $\begin{array}{l}\text { Traveler returning } \\
\text { from Morocco }\end{array}$ & $62 / \mathrm{M}$ & $\mathrm{P}$ & $\begin{array}{l}\text { Methotrexate } \\
\text { ustekinumab }\end{array}$ & 4 & $\mathrm{CL}$ & Pos SB & Pos SB & ND & $\begin{array}{l}\text { Intralesional } \\
\text { meglumine antimoniate } \\
\text { oral } \\
\text { miltefosine for } 28 \text { days }\end{array}$ & Yes & Cured \\
\hline $\begin{array}{l}\text { Guarneri et al., 2017, } \\
\text { Wien Med Wochenschr }\end{array}$ & $\begin{array}{l}\text { Endemic area } \\
\text { Italy }\end{array}$ & $65 / \mathrm{M}$ & PSA & $\begin{array}{l}\text { Golimumab cyclosporina } \\
\text { metotrexate } \\
\text { steroids }\end{array}$ & 48 & $\mathrm{ML}, \mathrm{VL}$ & ND & $\begin{array}{l}\text { posBM } \\
\text { posSB }\end{array}$ & Pos & $\begin{array}{l}\text { Liposomal amphotericin } \\
\text { B }\end{array}$ & Yes & Cured \\
\hline $\begin{array}{l}\text { Hernandez Bel et al., } \\
\text { 2018, Arch Soc Esp } \\
\text { Oftalmol }\end{array}$ & $\begin{array}{l}\text { Endemic area } \\
\text { Spain }\end{array}$ & $72 / \mathrm{M}$ & PSA & $\begin{array}{l}\text { Prednison } \\
\text { metotrexate } \\
\text { adalimumab }\end{array}$ & 60 & $\mathrm{CL}$ & Pos & Pos & ND & $\begin{array}{l}\text { Intralesional injections } \\
\text { of meglumine } \\
\text { antimoniate }\end{array}$ & Yes & Cured \\
\hline $\begin{array}{l}\text { Martinez-Domenech et } \\
\text { al., 2019, Br J Dermatol } \\
\text { (four patients) }\end{array}$ & $\begin{array}{l}\text { Endemic area } \\
\text { Spain }\end{array}$ & $\begin{array}{l}\text { 1 pt 34/F } \\
\text { 1pt40/F } \\
\text { 1pt 71/M } \\
1 \text { pt 53/M }\end{array}$ & $\begin{array}{l}\mathrm{P}(3) \\
\mathrm{PSA}(1)\end{array}$ & $\begin{array}{l}\text { Adalimumab (4) } \\
\text { Etanercept (2) } \\
\text { Metotrexate (2) }\end{array}$ & $\begin{array}{l}17 \\
96 \\
2 \\
24\end{array}$ & CL (4) & $\begin{array}{l}\text { ND (1) } \\
\operatorname{pos} S B(3)\end{array}$ & $\begin{array}{l}\operatorname{pos} \mathrm{SB}(2) \\
\text { neg } \mathrm{SB}(2)\end{array}$ & $\operatorname{neg}(4)$ & $\begin{array}{l}\text { Intralesional injections } \\
\text { of meglumine } \\
\text { antimoniate (4) }\end{array}$ & $\begin{array}{l}\text { Yes (3) } \\
\text { No (1) }\end{array}$ & $\begin{array}{l}\text { Cured } \\
(4)\end{array}$ \\
\hline $\begin{array}{l}\text { Caroleo et al., 2019, Curr } \\
\text { Drug Saf }\end{array}$ & $\begin{array}{l}\text { Endemic area } \\
\text { Italy }\end{array}$ & $48 / \mathrm{M}$ & PSA & $\begin{array}{l}\text { Prednisone } \\
\text { Metotrexate } \\
\text { cyclosporine Adalimumab }\end{array}$ & 12 & $\mathrm{CL}$ & ND & Pos SB & ND & $\begin{array}{l}\text { Intralesional injections } \\
\text { of meglumine } \\
\text { antimoniate }\end{array}$ & Yes & Cured \\
\hline Present case & $\begin{array}{l}\text { Endemic area } \\
\text { Italy }\end{array}$ & $46 / \mathrm{M}$ & $\mathrm{P}$ & Methotrexate & 180 & $\mathrm{VL}, \mathrm{MCL}$ & $\begin{array}{l}\text { pos SB pos } \\
\text { PB }\end{array}$ & ND & Pos & $\begin{array}{l}\text { Liposomal amphotericin } \\
\text { B }\end{array}$ & Yes & Cured \\
\hline
\end{tabular}

Number in parentheses indicates the number of patients of each article reporting the characteristic of the column header. M, male; F, female; P, psoriasis; PSA, psoriatic arthritis; CL, cutaneous leishmaniasis; VL, visceral leishmaniasis; ML, mucosal leishmaniasis; MCL, mucocutaneous leishmaniasis; pos, positive; neg, negative; SB, skin biopsy; BM, bone marrow; PB, peripheral blood; ND, not done. 
establishes chronic intracellular parasitism, survives for an infected person's lifetime and, in the event of major immune deficiency, may be reactivated from sites of latency. Leishmaniasis can present with a spectrum of clinical manifestations and three patterns of infection are described: cutaneous (CL), mucosal or mucocutaneous (ML or MCL) and visceral leishmaniasis (VL). The infecting species of Leishmania is very important in determining the clinical manifestations and the host immune response is crucial in determining the clinical outcome of infection ${ }^{2}$. Today, non-HIV related immunosuppressive conditions are becoming increasingly prevalent, mainly because of better medical care of patients with chronic illnesses and the therapeutic use of immunosuppressive drugs. In the field of rheumatology, leishmaniasis has been reported in association with the use of various immunosuppressive drugs. ${ }^{3}$ The introduction of tumor necrosis factor-alpha (TNF- $\alpha$ ) antagonist drugs has received much attention recently and several cases of VL have been reported in rheumatic patients who do anti-TNF $\alpha$ drugs. ${ }^{4}$

Psoriasis is a chronic inflammatory autoimmune disease affecting $2-3 \%$ of the world's population and characterized by an aberrant hyper-proliferation of keratinocytes. The pathogenesis of psoriasis is complex. Genetic susceptibility, environmental triggering factors and an over-reaction of local innate immune response initiate inflammation. Subsequent involvement of adaptive immune response with production of $\mathrm{Th}^{1}$ cytokines, chemokines and growth factors lead to epidermal hyperplasia. ${ }^{5}$

Recently, a functional role of interleukin-17-producing $\mathrm{T}$ helper cells (Th17) in psoriasis has been suggested by their reduction during successful anti-TNF treatment. ${ }^{6}$ It is also known that Th17 lymphocytes play an essential role in protecting against intracellular protozoa and in the successful clearance of Leishmania by strengthening the Th1 response. ${ }^{7}$ In view of this, it could be argued that psoriasis may represent a protective factor for Leishmania infection. Indeed, in our review we did not found any case of leishmaniasis in psoriatic subjects who were not under immunosuppressive therapies.

Biological agents, which are powerful immunosuppressive drugs, have been more and more used in rheumatic patients and Leishmania infections have been reported among anti-TNF-agents users. $^{8}$ Recently Maritati et al. found higher prevalence of subclinical leishmaniasis in patients with inflammatory rheumatic diseases receiving biological drugs than those treated with other immunosuppressive drugs. ${ }^{9}$ However, leishmaniasis has also been reported in psoriatic patients not receiving biological drugs, as occurred to our patient (Table 1 ).

Diagnosis of CL in psoriatic patients is challenging, as it mimics many other infections or a flare-up of psoriasis itself that can lead to ineffective and harmful changes of therapy. Immunosuppressive therapies cause atypical manifestations of leishmaniasis with large lesions spread over large cutaneous areas and associated to a possible mucosal involvement. ML by L. infantum is very rare and only sporadically described in patients receiving powerful immunosuppressive therapies or in HIV-coinfected patients. MCL is mostly observed in Latin America where L. braziliensis accounts for most cases, but L. panamensis, L. guyanensis, and L. amazonensis have also been implicated. Only rarely cutaneous lesions extend to areas of skin distant from the mucosa involved, as in our case in which two lesions on the foot and knee were associated with the oral lesion. In the context of impaired immunity, it is also advisable to rule out VL by PCR-Leismania on peripheral blood so as to establish the most appropriate therapy: intralesional or intravenous.

Finally, there is no agreement on appropriate screening for leishmaniasis before immunosuppressive treatments and on the strategy to be followed after the diagnosis of leishmaniasis in rheumatic patients taking immunosuppressive drugs. ${ }^{10}$ Molecular methods are highly sensitive and specific tools for the diagnosis of visceral leishmaniasis and a screening with Leishmania-PCR in immunosuppressed patients living in endemic areas could be useful to identify patients at highest risk of reactivation. ${ }^{9}$

Specific leishmaniasis treatment followed by suspension of the immunosuppressive therapy was adopted by most of the authors. Overall even if the treatment response is not as good as seen in the immunocompetent population, our review reports a good outcome in all cases and patients remained relapse-free without maintenance therapy and despite the ongoing use of immunosuppressive medication.

In conclusion physicians must be alert to the possibility of development of leishmaniasis in immunosuppressed rheumatic patients. Adequate screening for VL should be incorporated into the list of baseline studies to carry out before initiating biologic therapies, at least in endemic areas.

\section{Declaration of Competing Interest}

The authors declare that there is no conflict of interest.

\section{Acknowledgment}

This research did not receive any specific grant from funding agencies in the public, commercial, or not-for-profit sector.

\section{Supplementary materials}

Supplementary material associated with this article can be found, in the online version, at doi:10.1016/j.jinf.2020.01.019.

\section{References}

1. Brunet K., Alanio A., Lortholary O., Rammaert B.. Reactivation of dormant/latent fungal infection. J Infect 2018;77:463-8. doi:10.1016/j.jinf.2018.06.016.

2. Saporito L., Giammanco G.M., De Grazia S., Colomba C.. Visceral leishmaniasis: host-parasite interactions and clinical presentation in the immunocompetent and in the immunocompromised host. Int J Infect Dis 2013;17:e572-6. doi:10.1016/j.ijid.2012.12.024.

3. Erre G.L., Mesina P., Tonelli N., Passiu G.. Visceral Leishmaniasis among immunosuppressed patients with rheumatic diseases. Clin Exp Rheumatol 2010;28:590-1.

4. Zanger P., Kotter I., Kremsner P.G., Gabrysch S.. Tumor necrosis factor alpha antagonist drugs and leishmaniasis in Europe. Clin Microbiol Infect 2012;18:670-6. doi:10.1111/j.1469-0691.2011.03674.x.

5. Grozdev I., Korman N., Tsankov N.. Psoriasis as a systemic disease. Clin Dermatol 2014;32:343-50. doi:10.1016/j.clindermatol.2013.11.001.

6. Deng Y., Chang C., Lu Q.. The inflammatory response in psoriasis: a comprehensive review. Clin Rev Allergy Immunol 2016;50:377-89. doi:10.1007/ s12016-016-8535-X.

7. Gonçalves-de-Albuquerque S.D.C., Pessoa-E-Silva R., Trajano-Silva L.A.M., de Goes T.C., de Morais R.C.S., da C Oliveira C.N., et al. The Equivocal role of Th17 cells and neutrophils on immunopathogenesis of leishmaniasis. Front Immunol 2017;8:1437. doi:10.3389/fimmu.2017.01437.

8. Cascio A., Iaria M., Iaria C.. Leishmaniasis and biologic therapies for rheumatologic diseases. Semin Arthritis Rheum 2010;40:e3-5. doi:10.1016/j.semarthrit. 2009.07.001.

9. Maritati M., Trentini A., Michel G., Bellini T., Almugadam S., Hanau S., et al. Subclinical Leishmania infection in patients with rheumatic diseases under biological drugs. Infection 2018;46:801-9. doi:10.1007/s15010-018-1189-2.

10. Cascio A., Iaria C.. Appropriate screening for leishmaniasis before immunosuppressive treatments. Emerg Infect Dis 2009;15:1706 author reply -7. doi:10.3201/ eid1510.090881.

Claudia Colomba, Laura Saporito, Silvia Bonura Department of Health Promotion, Mother and Child Care, Internal Medicine and Medical Specialties "G. D'Alessandro", University of Palermo, Via Liborio Giuffrè, Palermo, Italy

Giuseppina Campisi Department of Surgical, Oncological and Stomatological Disciplines, University of Palermo, Via Liborio Giuffrè, Palermo, Italy 
Paola Di Carlo

Department of Health Promotion, Mother and Child Care, Internal Medicine and Medical Specialties "G. D'Alessandro", University of Palermo, Via Liborio Giuffrè, Palermo, Italy

Vera Panzarella Department of Surgical, Oncological and Stomatological Disciplines, University of Palermo, Via Liborio Giuffrè, Palermo, Italy

Valentina Caputo, Antonio Cascio Department of Health Promotion, Mother and Child Care, Internal Medicine and Medical Specialties "G. D'Alessandro", University of Palermo, Via Liborio Giuffrè, Palermo, Italy

*Corresponding author. E-mail addresses: claudia.colomba@libero.it (C. Colomba), laura.sapo@tin.it (L. Saporito), giuseppina.campisi@unipa.it (G. Campisi), paola.dicarlo@unipa.it (P. Di Carlo), panzarella@odonto.unipa.it (V. Panzarella), valentina.caputo@unipa.it (V. Caputo), Antonio.cascio03@unipa.it

(A. Cascio)

Accepted 29 January 2020 Available online 15 January 2020

https://doi.org/10.1016/j.jinf.2020.01.019

(C) 2020 The British Infection Association. Published by Elsevier Ltd. All rights reserved.

\section{Challenges to develop an equine herpesvirus vaccine in China}

Dear Editors,

As demonstrated in several studies in Journal of infection, herpesviruses pose an increasing threat to human health. ${ }^{1-3}$ According to International Committee on Taxonomy of Viruses (ICTV), equine herpesviruses (EHVs) belong to the family Herpesviridae. Until now, a total of 9 EHV species types have been determined in equines, viz. EHV1-EHV9. ${ }^{4}$ Among them, EHV1 and EHV4 are the most relevant herpesviruses affecting equines. Both EHV1 and EHV4 infection are associated with upper respiratory tract disease, but only EHV1 infection could cause abortion and myeloencephalitis. EHV1 and EHV4 are prevalent in equines on all continents and have considerable economic impact on the horse industry. ${ }^{5}$

In China, the number of equines is very large, reaching to be $~$ 6.8 million in 2018 (http://www.stats.gov.cn/tjsj/zxfb/). EHV infection in equines was first reported in China in 1980, and the epidemiological investigation since then indicated EHV was prevalent in the equine population in all the studied 16 provinces in Mainland China, with a seroprevalence ranging $8 \%-92 \%{ }^{6-8}$ Vaccination is commonly used to prevent and control EHV. However, China has not developed a commercially available EHV vaccine so far.

EHV vaccine has a limited market application potential in China currently. Due to the lack of relevant knowledge on EHV, most of the Chinese horse owners always erroneously identified it as other common pathogen of equine respiratory diseases, and didn't realize its potential threat to equine health and reproduction. Although the number of equines in China is large, most of them are labor/farming horses. To the best of our knowledge, even for racehorses, vaccination with EHV vaccine has not been performed in Mainland China. Considering the wide distribution and high prevalence of EHV in China, it is urgently to popularize knowledge on EHV in horse owners and promote market application prospects of EHV vaccine.
In China, few veterinary researchers are currently investigating equine virus, including EHV. This is mainly caused by the change of equines' historical role. In the last century, a great number of equines were used for military in China. However, there is only one military equine farm in Mainland China at present. Considering a more important economic role of other domestic animals (e.g., pigs, chickens, and cattle) compared with equines, investigating equine virus (including EHV) is not a priority in the related guide policies issued by the Chinese government.

Though epidemiological studies on EHV in China are limited, it still could be concluded that epidemic status of EHV is very complicated in China, which increases the difficulty in EHV vaccine development. In most provinces, EHV1 and EHV4 were co-circulating in equines with a high seroprevalence. ${ }^{8}$ Until now, a total of 7 EHV1 strains have been isolated from tissue samples of aborted equine fetuses ( 5 from farming horses in Northeast China in 1980, 1 from Asian wild horses in Western China in 1999, 1 from farming horses in Western China in 2016). ${ }^{6,8}$ In addition, a novel EHV8 strain was isolated from one horse with serious respiratory disease in Northern China in $2010 .^{9}$ Recently, our laboratory firstly determined the molecule evinces for EHV4 and EHV5 in racehorses in Sothern China (data not shown). However, a more large-scale and surveillance of EHV in equines is necessary to fully understand epidemic status of EHV in China, which could establish a foundation for updating the composition of EHV vaccine developed in China in future.

In other countries, much effort has been made to develop EHV vaccine, and modified-live and inactivated virus vaccines have been registered for sale. ${ }^{10}$ Before an EHV vaccine is developed successfully in China by itself, it is necessary to vaccinate the susceptible equine population with an EHV vaccine commercially available from other countries to prevent and control EHV in China. However, a well-designed case-control animal challenge study still needs to estimate the protective efficacy of different vaccines against the field prevalent EHV strains in China.

\section{Declaration of Competing Interest}

All authors declare that they have no competing interests.

\section{Acknowledgements}

This work was supported by the National Natural Science Foundation of China (31702271), and the Guangdong Provincial Natural Science Foundation (2017A030310367).

\section{References}

1. Balsat M., Pillet S., Tavernier E., Cacheux V., Escuret V., Chabrot C.M., et al. Human herpesvirus 6 infection after autologous stem cell transplantation: a multicenter prospective study in adult patients. J Infect 2019;79:36-42.

2. Zhang J.Z., Zheng P., Sun H.M., Dong J.J., Li S.L., Fan S.Y., et al. Next-generation sequencing combined with routine methods to detect the pathogens of encephalitis/meningitis from a Chinese tertiary pediatric neurology center. J Infect 2019;78:409-21.

3. Maple P.A.C., Tanasescu R., Gran B., Constantinescu C.S.. A different response to cytomegalovirus (CMV) and Epstein-Barr virus (EBV) infection in UK people with multiple sclerosis (PwMS) compared to controls. J Infect 2019.

4. Fukushi H., Yamaguchi T., Yamada S. Complete genome sequence of equine herpesvirus type 9. J Virol 2012:86:13822-13822.

5. Ma G.G., Azab W., Osterrieder N. Equine herpesviruses type 1 (EHV-1) and 4 (EHV-4)-Masters of co-evolution and a constant threat to equids and beyond. Vet Microbiol 2013;167:123-34

6. Liu J., Hao C., Yuan Z. Isolation and identification of equine herpesvirus type 1. J Microbiol 1982;22:88-94 in Chinese.

7. Lu J., Li X., Ge F., Yang D., Shen H., Ju H., et al. Identification of equine herpesvirus type 1 infection in a horse farm in Shanghai. Chin J Anim Quarantine 2018;36:86-8 in Chinese.

8. Luo A., Zhang Y., Diao Q., Gong F., Lu G., Yu H., et al. Epidemiological status of equine rhinopneumonia in China. Chin J Veterinary Med 2016;52:84-7 in Chinese. 
9. Liu C., Guo W., Lu G., Xiang W., Wang W.. Complete genomic sequence of an equine herpesvirus type 8 Wh strain isolated from China. J Virol 2012;86:5407.

10. Patel J.R., Heldens J.. Equine herpesviruses 1 (EHV-1) and 4 (EHV-4)-epidemiology, disease and immunoprophylaxis: a brief review. Veterinary J 2005;170:14-23.

Chihai Ji, Siqi Cai College of Veterinary Medicine, South China Agricultural University, Guangzhou, Guangdong Province 510642, China Key Laboratory of Zoonosis Prevention and Control of Guangdong Province, Guangzhou, Guangdong Province 510642, China Guangdong Laboratory for Lingnan Modern Agriculture, Guangzhou, 510642, China

Gang Lu*

College of Veterinary Medicine, South China Agricultural University, Guangzhou, Guangdong Province 510642, China Guangdong Laboratory for Lingnan Modern Agriculture, Guangzhou, 510642, China

Guangdong Provincial Key Laboratory of Prevention and Control for Severe Clinical Animal Diseases, Guangzhou, Guangdong Province 510642, China Guangdong Technological Engineering Research Center for Pet, Guangzhou, Guangdong Province 510642, China

Guihong Zhang*

College of Veterinary Medicine, South China Agricultural University, Guangzhou, Guangdong Province 510642, China Key Laboratory of Zoonosis Prevention and Control of Guangdong Province, Guangzhou, Guangdong Province 510642, China Guangdong Laboratory for Lingnan Modern Agriculture, Guangzhou, 510642, China

*Corresponding authors. E-mail addresses: LG@scau.edu.cn (G. Lu), guihongzh@scau.edu.cn (G. Zhang)

Accepted 18 January 2020 Available online 15 January 2020

https://doi.org/10.1016/j.jinf.2020.01.013

(c) 2020 The British Infection Association. Published by Elsevier Ltd. All rights reserved.

\section{Comparative hepatitis $C$ genotype 1-3 viral load kinetics in response to directly-acting antiviral therapy}

Dear editor,

A recent review article on the treatment of hepatitis $C$ with directly-acting antiviral (DAA) drugs, makes numerous recommendations for baseline drug resistance testing. ${ }^{1}$ In our local practice, we have been performing baseline drug resistance testing for some years now, prior to the publication of these guidelines. We present a recent retrospective HCV kinetics analysis of these patients' changing viral loads in response to DAA therapy below. Such studies have been used previously to compare viral suppressive responses in different HCV genotypes and treatment regimens. ${ }^{2,3}$ The patients were a mixture of treatment-naive and treatment-experienced (including with interferon-based, NS3 protease inhibitor-based and DAA-based regimens) cases.

The current standard of care for hepatitis (HCV) patients is a combination of direct acting antivirals (DAAs), for which there are three different HCV viral protein targets (NS3, NS5A and NS5B).
Table 1

NS3, NS5A, NS5B resistance associated substitutions (RAS), by HCV genotype, found in this patient cohort at baseline drug resistance testing (viral sequencing performed at Imperial College, London, UK). The patients included a mixture of treatment-naïve and treatment experienced (i.e. interferon-based, NS3 protease inhibitor-based and more recent DAA-based regimens) cases.

\begin{tabular}{ll}
\hline DAA target gene & Resistance associated substitution (RAS) by HCV genotype \\
\hline NS3 & G1a: $54 \mathrm{~S}, 55 \mathrm{~A}, 80 \mathrm{~K}, 122 \mathrm{G}, 122 \mathrm{~N}, 168 \mathrm{~V}, 170 \mathrm{~V}, 174 \mathrm{~A}, 174 \mathrm{~B}$, \\
& 174 N, $174 \mathrm{~S}$ \\
& G1b: $36 \mathrm{M}, 80 \mathrm{~K}, 170 \mathrm{I}, 170 \mathrm{~V}$ \\
& G3a: $170 \mathrm{I}$ \\
& G1a: $28 \mathrm{~T}, 28 \mathrm{~V}, 30 \mathrm{H}, 30 \mathrm{R} .58 \mathrm{P}, 93 \mathrm{H}$ \\
NS5A & G1b: $31 \mathrm{M}, 93 \mathrm{H}$ \\
& G3a: $30 \mathrm{~K}, 93 \mathrm{H}$ \\
& G3b:30 K, $31 \mathrm{M}$ \\
& G4a: $28 \mathrm{M}$ \\
& G1a: $444 \mathrm{D}, 556 \mathrm{G}$ \\
NS5B & G1b: $316 \mathrm{~N}$ \\
& G2a: $289 \mathrm{R}$ \\
& G4a: $282 \mathrm{~S}$ \\
\hline
\end{tabular}

Treatment with DAAs cure the vast majority of HCV-infected patients, with oral regimens having $>95 \%$ efficacy in most patient groups. ${ }^{1,4,5}$ Treatment failure currently affects approximately $5 \%$ of treated patients and is often associated with the selection of resistance associated substitutions (RAS). ${ }^{1}$

We performed HCV drug resistance testing both retrospectively (following treatment failures) and prospectively (prior to treatment) in our cohort of HCV genotype (G) 1-3-infected patients, during March 2015-June 2018. Viral extraction, PCR and sequencing were performed at Imperial College, using Qiagen Viral RNA mini kits (Qiagen PN:52906, Qiagen Ltd., Manchester, UK), and inhouse PCR and Sanger sequencing methods on an ABI Prism 3100Avant genetic analyser (Thermo Fischer Scientific, Loughborough, UK). The prediction of HCV genotype and drug sensitivities is derived from the geno2pheno algorithm [www.geno2pheno.org]. ${ }^{6}$

Treatment regimens used during this period complied with contemporaneous NHS rate cards: for non-cirrhotic or compensated cirrhotic patients: G1-treatment-naive: OMB/PAR/RIT+ DAS+R; G1-treatment-experienced: ELB/GRZ+/-R; G2-treatmentnaive/experienced: GLE/PIB; G3-treatment-naive/experienced: GLE/ PIB; G1-decompensated cirrhotic patients SOF/LED+R; G2/G3decompensated cirrhotic patients: SOF/VEL $+R$.

We assessed the impact of any RAS across G1-G3 on HCV RNA kinetics by analysing viral load (RealTime HCV Viral Load, Abbott m2000, Abbott Molecular UK, Maidenhead, England) decline rates. We applied linear mixed regression to model the viral loads and assumed a linear decline ( $\log _{10}$ scale) over time, using SAS statistical software (SAS Institute Inc., NC, USA).

In this cohort of 54 patients ( $\mathrm{n}: \mathrm{G} 1=21, \mathrm{G} 2=6, \mathrm{G} 3=27$ ), HCV RAS were found as shown in Table 1. HCV RNA viral load decline rates were found to be similar and not statistically different $(p=0.760)$ at: $-2 \log _{10}$ and $-1.8 \log _{10}$ per month, respectively, for G1 and G2/G3 (Fig. 1). This suggests that these viral load decline rates were similar across G1-G3 infections despite baseline differences in viral load, RAS profile, or a history of any previous treatment (i.e. interferon-based, older NS3 protease inhibitor-based, or more recent DAA regimens).

These results demonstrate similar HCV RNA clearance efficacies of the various DAA treatment regimens for $\mathrm{G} 1-\mathrm{G} 3$, in this patient cohort. Although other studies on HCV kinetics have been published, they do not usually compare multiple HCV genotypes. ${ }^{7}$ Similar studies on patients infected with G4-6 viruses, and/or undergoing other DAA treatment or retreatment combinations, ${ }^{8,9}$ will be 


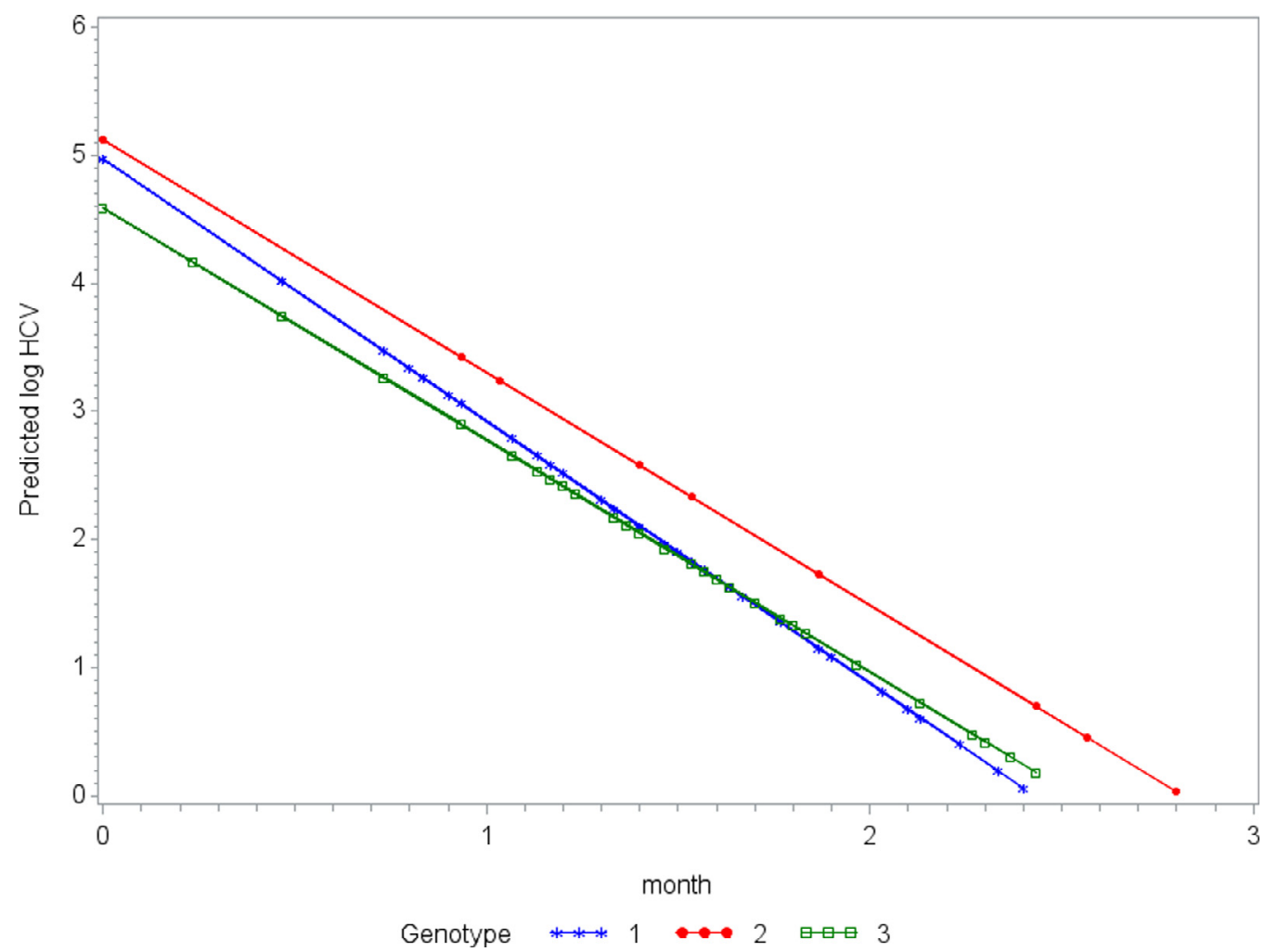

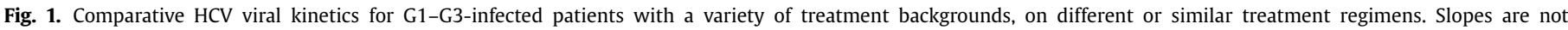
significantly different $(p=0.760)$, ranging from $-2 \log _{10}$ to $-1.8 \log _{10}$ per month, respectively, for $\mathrm{G} 1$ and G2/G3.

useful to determine if such clearance efficacies are maintained for all DAA treatment regimens across all HCV genotypes, regardless of any previous treatment history.

\section{References}

1. Bradshaw D., Mbisa J.L., Geretti A.M., Healy B.J., Cooke G.S., Foster G.R., et al. UK. Consensus recommendations for resistance testing in the management of chronic hepatitis c virus infection: public health England HCV resistance group. J Infect 2019;79(December (6)):503-12. doi:10.1016/j.jinf.2019.10.007.

2. Neumann A.U., Lam N.P., Dahari H., Davidian M., Wiley T.E., Mika B.P., et al. Differences in viral dynamics between genotypes 1 and 2 of hepatitis C virus. J Infect Dis 2000;182(July (1)):28-35.

3. Nguyen T.H.T., Guedj J., Uprichard S.L., Kohli A., Kottilil S., Perelson A.S.. The paradox of highly effective sofosbuvir-based combination therapy despite slow viral decline: can we still rely on viral kinetics? Sci Rep 2017;7(August (1)):10233. doi:10.1038/s41598-017-09776-z.

4. Conteduca V., Sansonno D., Russi S., Pavone F., Dammacco F.. Therapy of chronic hepatitis $C$ virus infection in the era of direct-acting and host-targeting antiviral agents. J Infect 2014;68(January (1)):1-20. doi:10.1016/j.jinf.2013.08.019.

5. Falade-Nwulia O., Suarez-Cuervo C., Nelson D.R., Fried M.W., Segal J.B., Sulkowski M.S.. Oral direct-acting agent therapy for hepatitis $C$ virus infection: a systematic review. Ann Intern Med 2017;166(May (9)):637-48. doi:10.7326/ M16-2575.

6. Kalaghatgi P., Sikorski A.M., Knops E., Rupp D., Sierra S., Heger E., et al. Geno2pheno[HCV] - a Web-based interpretation system to support hepatitis $C$ treatment decisions in the era of direct-acting antiviral agents. PLOS ONE 2016;11(May (5)):e0155869. doi:10.1371/journal.pone.0155869.

7. Nakamoto S., Kanda T., Shirasawa H., Yokosuka O.. Antiviral therapies for chronic hepatitis C virus infection with cirrhosis. World J Hepatol 2015;7(May (8)):113341. doi:10.4254/wjh.v7.i8.1133.

8. Sanai F.M., Altraif I.H., Alswat K., AlZanbagi A., Babatin M.A., AlMousa A., et al. Real life efficacy of ledipasvir/sofosbuvir in hepatitis C genotype 4-infected patients with advanced liver fibrosis and decompensated cirrhosis. $J$ Infect 2018;76(June (6)):536-42. doi:10.1016/j.jinf.2018.04.001.

9. Due O.T., Chaikledkaew U., Genuino A.J.M., Sobhonslidsuk A., Thakkinstian A.. Systematic review with meta-analysis: efficacy and safety of directacting antivirals for chronic hepatitis $\mathrm{C}$ genotypes 5 and 6. Biomed Res Int 2019;2019(November):2301291. doi:10.1155/2019/2301291.
Shoshanna May Clinical Microbiology, University Hospitals of Leicester NHS Trust, Leicester, United Kingdom

Feza Kurmoo, Mariesa Iliffe Infectious Diseases Unit, University Hospitals of Leicester NHS Trust, Leicester, United Kingdom

Joicy David, Ayushi Patel Clinical Microbiology, University Hospitals of Leicester NHS Trust, Leicester, United Kingdom

Martin Wiselka, Iain Stephenson Infectious Diseases Unit, University Hospitals of Leicester NHS Trust, Leicester, United Kingdom Respiratory Sciences, University of Leicester, Leicester, United Kingdom

Toby Delahooke

Hepatology, University Hospitals of Leicester NHS Trust, Leicester, United Kingdom

Florence Y Lai Cardiovascular Science, University of Leicester, Leicester, United Kingdom

Simon Dustan

Molecular Diagnostics Unit, Imperial College, London, United Kingdom

Julian W Tang*

Clinical Microbiology, University Hospitals of Leicester NHS Trust, Leicester, United Kingdom Respiratory Sciences, University of Leicester, Leicester, United Kingdom 
*Correspondence author at: Clinical Microbiology, University Hospitals of Leicester NHS Trust, Level 5 Sandringham Building, Leicester Royal Infirmary, Infirmary Square, Leicester LE1 5WW, United Kingdom. E-mail address: julian.tang@uhl-tr.nhs.uk (J.W. Tang)

Accepted 18 January 2020 Available online 15 January 2020

\section{https://doi.org/10.1016/j.jinf.2020.01.011}

(c) 2020 The British Infection Association. Published by Elsevier Ltd. All rights reserved.

High prevalence of Bordetella pertussis in young hospitalized infants with acute respiratory infection in the south of China: age- and season-dependent effects

\section{Dear Editor,}

With great interest we have read the report of Zhang et al. concerning the increased susceptibility to pertussis in Chinese adults at childbearing age, as determined in a comparative seroprevalence study using samples collected from 2010 to 2016. ${ }^{1}$ The authors describe that about $5 \%$ of the individuals had PT-IgG antibodies, which is indicative of a recent infection. In the adults 20-39 years of age, 29.1\% subjects had undetectable PT-IgG antibodies in 2010 but $57.4 \%$ in $2015 / 2016$. It is well known that adolescents and adults have become the reservoir of pertussis and an important source of transmission to vulnerable infants. Bordetella pertussis is commonly associated with atypical pneumonia as determined in hospitalized children. ${ }^{2}$ Several seroprevalence studies conducted in different regions of China indicate that the incidence of pertussis is most likely underestimated. ${ }^{3,4}$ This may be due to the use of insensitive diagnostics. At present, the diagnosis of pertussis in China is mainly based on culture. However, both the CDC and the World Health Organization (WHO) use PCR as the gold standard for diagnosis, in addition to culture. ${ }^{3}$

Oropharyngeal or nasopharyngeal swabs were obtained from 17,104 inpatients aged between 10 days and 14 years of age with clinical suspicion of pertussis, enrolled from March 2014 to February 2018 in Shenzhen Children's Hospital. More than 50\% of all patients were younger than 6 months of age. The hospitalized

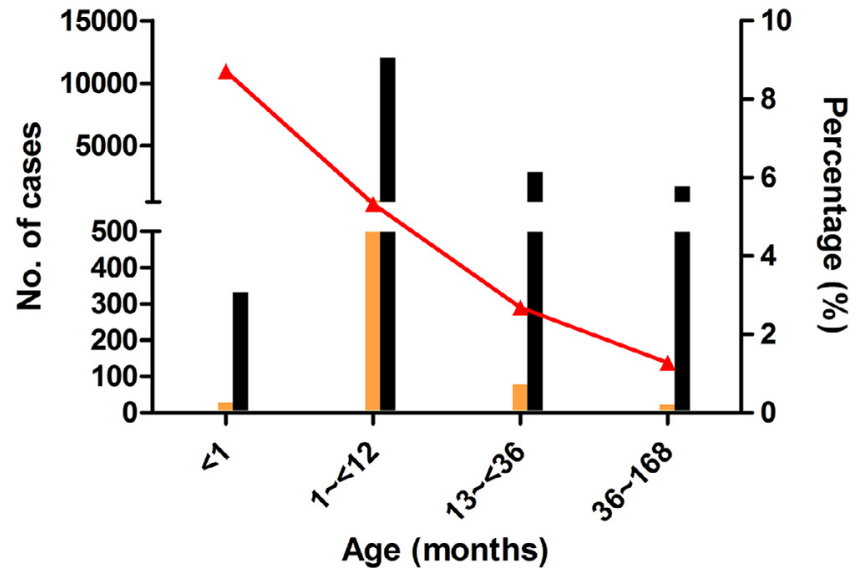

Fig. 1. B. pertussis infection rates in children with acute lower respiratory tract infections admitted at the Shenzhen Children's Hospital. The black bars indicate the total number of acute respiratory tract infection cases in the different age groups, the orange bars indicate the number of $B$. pertussis cases (left Y-axis). The red line indicates the percentage of $B$. pertussis cases (right Y-axis).

patients included 10,894 males and 6210 females (sex ratio, 1.75). All patients recovered after the treatment.

A real time PCR assay targeting $p t x A-P r$ was used to detect $B$. pertussis. Of the 17,104 samples, 772 (4.51\%) tested positive for $B$. pertussis by RT-PCR. Our results indicate that despite vaccination pertussis remains a major health problem in China, since the prevalence of infection by $B$. pertussis in hospitalized children was high. The majority of patients were admitted because of pneumonia. The detection rate in hospitalized patients was lower than the rates reported earlier in Shanghai and Ji'nan. ${ }^{3,4}$ This may be due to lower number of samples collected in these studies and due to the use of serology or culture methods.

The overall prevalence rates were $3.97 \%$ and $5.48 \%$, respectively. However, $B$. pertussis infection in female patients was significantly higher than in male patients $\left(X^{2}=20.913, p<0.001\right)$. This has earlier been reported by the $\mathrm{ECDC}^{5}$ and Haberling et al. ${ }^{6}$ and may point to a genetic association with susceptibility to $B$. pertussis.

The detection rates were dependent on age in patients $\left(X^{2}=139.8, p<0.001\right)$. The prevalence decreased with age: $8.71 \%$ newborns, $5.33 \%$ in infants, $2.39 \%$ in toddlers, $1.28 \%$ in (pre-) schoolers (Fig. 1). The high vulnerability of newborns and young infants for $B$. pertussis infection may be related to a combination

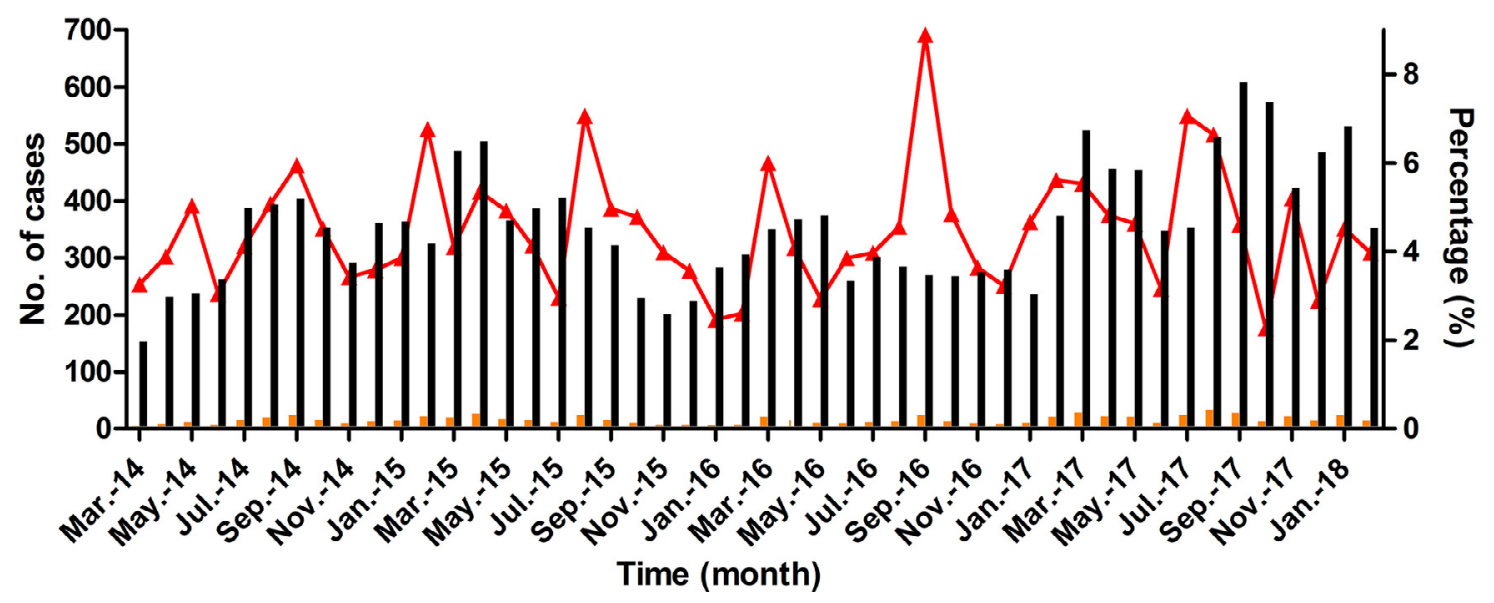

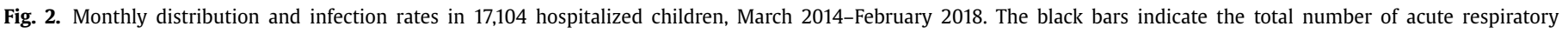

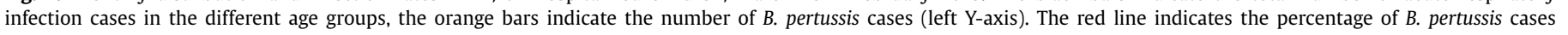
(right Y-axis). 
of insufficient herd immunity and suboptimal protection against B. pertussis infection in children too young to be fully vaccinated. Since vaccination rates in infants are already at $99 \%$, it will be difficult to improve this further. Therefore, other measures must be considered, including booster vaccination at pre-school age and vaccination during pregnancy. Because young infants are mainly cared for by mothers and other adults, the most important cause of infection with B. pertussis is their close contact with parents and siblings. ${ }^{7}$

In general, $B$. pertussis was detected more often during seasonal changes, especially from late summer to early autumn. In hospitalized children the number of $B$. pertussis infections increased in March and September as compared to other months (Fig. 2). The seasonal infection rates were $4.66 \%$ in spring, $4.75 \%$ in summer, $4.62 \%$ in autumn and $4.00 \%$ in winter, respectively. The prevalence in the winter season was lower but not statistically different than in other seasons $\left(\mathrm{X}^{2}=3.388, p=0.336\right)$.

In this study, we used real-time PCR, the most accurate method to detect $B$. pertussis. The detection rate may be significantly lower than the actual level, because oropharyngeal samples in most patients were collected instead of nasopharyngeal samples, and the PCR target gene was $p t x A-P r$ instead of IS481. IS481 is present in high-copy numbers in $B$. pertussis whereas $p t x A-P r$ is a single-copy target. However, the ptxA-Pr PCR is more specific and will not detect $B$. parapertussis, which contributes to more than $5 \%$ of pertussis cases. ${ }^{8}$

Many studies have shown that adolescents and adults with B. pertussis infections, causing chronic cough, are an important reservoir for transmission, putting newborns at high risk. ${ }^{9}$ Maternal pertussis immunization prevents infant pertussis, as recently shown by Amirthalingam et al. Vaccine effectiveness against infant deaths was estimated at $95 \%$, and disease incidence in infants $<3$ months of age has remained low. ${ }^{10}$ According to our results, vaccination of pregnant women and adults, especially those in close contact with infants and young children, may help to prevent pertussis in infants and young children in China.

\section{Declaration of Competing Interest}

The authors have declared that no competing interests exist.

\section{Funding}

This study was supported by the Sanming Project of Medicine in Shenzhen (SZSM201512030) and by the Shenzhen Science and Technology Project (JCYJ20170303155012371).

\section{References}

1. Zhang Y., Chen Z., Zhao J., Zhang N., Chen N., Zhang J., Li S., He Q.. Increased susceptibility to pertussis in adults at childbearing age as determined by comparative seroprevalence study, China 2010-2016. J Infect 2019;79(July (1)):1-6.

2. Sadiasa A., Saito-Obata M., Dapat C.. RITM-Tohoku collaborative research group. Bordetella pertussis infection in children with severe pneumonia, Philippines, 2012-2015. Vaccine 2017;35(7):993-6

3. Li J., Tao Y., Tang M., Du B., Xia Y., Mo X.. Rapid detection of respiratory organisms with the Filmarray respiratory panel in a large children's hospital in China. BMC Infect Dis 2018;18(1):510.

4. Meng Q., Li L., Shi W., Wang Q., Ding M., Liu Y.. Seroprevalence of diphtheria and pertussis immunoglobulin $\mathrm{G}$ among children with pneumonia in Ji'nan. China. BMC Pediatr 2018 Dec 5;18(1):383.
5. European Centre for Diseases Prevention and Control. Annual epidemiological report 2015. Pertussis. Stockholm: ECDC; 2016.

6. Haberling D.L. Holman R.C., Paddock C.D. Murphy TV Infant and maternal risk factors for pertussis-related infant mortality in the United States, 1999 to 2004. Pediatr Infect Dis J 2009;28(3):194-8.

7. Fu P., Wang C., Tian H., Kang Z., Zeng M.. Bordetella pertussis infection in infants and young children in Shanghai, China, 2016-2017: clinical features, genotype variations of antigenic genes and macrolides resistance. Pediatr Infect Dis J. 2019;38(April (4)):370-6.

8. Liu X., Wang Z, Zhang J. Li F, Luan Y, Li $\mathrm{H}$, et al. Pertussis outbreak in a primary school in China: infection and transmission of the macrolide-resistant Bordetella pertussis. Pediatr Infect Dis J 2018;37(6):e145-8.

9. Chen Z., Zhang J., Cao L., Zhang N., Zhu J., Ping G., et al. Seroprevalence of pertussis among adults in China where whole cell vaccines have been used for 50 years. J Infect 2016;73(1):38-44.

10. Amirthalingam G., Campbell H., Ribeiro S., Fry N.K., Ramsay M., Miller E., et al. Sustained effectiveness of the maternal pertussis immunization program in England 3 years following introduction. Clin Infect Dis 2016;63(suppl 4):S236-43.

Heping Wang

Shenzhen Children's Hospital, Shenzhen, Guangdong, China Section Pediatric Infectious Diseases, Laboratory of Medical Immunology, Radboud Center for Infectious Diseases, Radboud University Medical Center, Nijmegen, The Netherlands

Yuejie Zheng

Shenzhen Children's Hospital, Shenzhen, Guangdong, China

Ronald de Groot

Section Pediatric Infectious Diseases, Laboratory of Medical Immunology, Radboud Center for Infectious Diseases, Radboud University Medical Center, Nijmegen, The Netherlands

Yonghong Yang

Shenzhen Children's Hospital, Shenzhen, Guangdong, China

Dimitri A. Diavatopoulos

Section Pediatric Infectious Diseases, Laboratory of Medical Immunology, Radboud Center for Infectious Diseases, Radboud University Medical Center, Nijmegen, The Netherlands

Yunsheng Chen Shenzhen Children's Hospital, Shenzhen, Guangdong, China Marien I. de Jonge** Section Pediatric Infectious Diseases, Laboratory of Medical Immunology, Radboud Center for Infectious Diseases, Radboud University Medical Center, Nijmegen, The Netherlands

Jikui Deng*

Shenzhen Children's Hospital, Shenzhen, Guangdong, China

*Corresponding author at: Shenzhen Children's Hospital, No. 7019 Yitian Road, Futian District, Shenzhen, Guangdong 518038, China. ** Corresponding author at: Radboud University Medical Center, Philips van Leydenlaan 15, PO Box 9101, 6500 HB Nijmegen, the Netherlands.

E-mail addresses: Marien.DeJonge@radboudumc.nl (M.I. de Jonge), szsetyydeng@sina.com (J. Deng)

Accepted 13 January 2020 Available online 15 January 2020

https://doi.org/10.1016/j.jinf.2020.01.009

0163-4453/@ 2020 Published by Elsevier Ltd on behalf of The British Infection Association. 


\section{Novel coronavirus disease (Covid-19): The first two patients in the UK with person to person transmission}

Tang and colleagues reported in this journal their experience with COVID-19 disease ${ }^{1}$, the outbreak of which began in December 2019 in Wuhan, Hubei province, China ${ }^{2,3}$ with spread to 33 additional countries ${ }^{4-8}$ as of the 21 st February 2020 . Here we report the clinical features and outcome of the first two cases of disease caused by SARS-CoV-2 infection in the United Kingdom (UK) - the first imported and the second associated with probable person-toperson transmission within the UK. Public health management will be reported separately.

The index case (A) entered the UK on 23/01/20 from Hubei province in China. Initially asymptomatic, this individual, a 50 year-old female with no past medical history and on no regular medications, developed symptoms of fever and malaise on $26 / 01 / 20$, accompanied by sore throat and dry cough. She had travelled with her partner and reported no infectious contacts prior to travel. On 28/01/20, a close household contact of the index case, a resident of the UK, developed symptoms of fever $\left(38.5^{\circ} \mathrm{C}\right)$, followed the next day by diffuse myalgia and a dry cough. This patient (case B) is a previously fit and well 23 year-old male. He had returned to the UK from Hubei province on 06/01/20.

Case B promptly sought advice via the National Health Service (NHS) self-referral service NHS 111, and he and case A were assessed as being possibly at risk of Covid-19, and were admitted to the regional infectious diseases unit at Castle Hill Hospital, Hull University Teaching Hospitals for isolation, assessment and diagnostic sampling. They were managed in separate negative pressure cubicles with anterooms. Nursing and medical staff donned personal protective equipment (PPE) as recommended by Public Health England (PHE). The clinical observations of each of the patients, together with their initial blood tests, are shown in Table 1.
Table 1

Baseline clinical parameters on admission (bloods on case A done on admission to HLIU).

\begin{tabular}{lll}
\hline Parameter & Case A & Case B \\
\hline Heart rate/min & 116 & 91 \\
Respiratory rate/min & 18 & 17 \\
Oxygen saturation (room air) & $97 \%$ & $97 \%$ \\
Blood pressure $(\mathrm{mmHg})$ & $120 / 80$ & $115 / 74$ \\
Temperature $\left({ }^{\circ} \mathrm{C}\right)$ & 37.6 & 37.0 \\
Total white cell count $\left(\times 10^{9} / \mathrm{l}\right)$ & 3.4 & 10.5 \\
Neutrophil count $\left(\times 10^{9} / \mathrm{l}\right)$ & 2.4 & 8.67 \\
Lymphocyte count $\left(\times 10^{9} / \mathrm{l}\right)$ & 0.6 & 1.1 \\
C-Reactive Protein $(\mathrm{mg} / \mathrm{L})$ & 15 & 40 \\
\hline
\end{tabular}

Diagnostic PCR testing of nasopharyngeal swabs using the BioFire FilmArray Respiratory Panel 2 plus (bioMérieux, Marcy l'Etoile, France) detected rhinovirus/enterovirus RNA in patient $B$ and was negative in patient A. SARS-CoV-2 RNA was detected by parallel PCR testing of nasopharyngeal swabs from cases $A$ and $B$ at the Public Health England (PHE) Respiratory Virus Unit, Colindale, London, and reported on 30/01/20. The NHS England Airborne High Consequence Infectious Disease (HCID) network was activated and the decision was made to transfer the patients by Hazardous Area Response Team ambulance to the HCID High-Level Isolation Unit (HLIU) at The Newcastle-upon Tyne NHS Foundation Trust in the early hours of $31 / 01 / 20$.

Upon arrival in the HLIU (d0) both patients remained clinically stable. Symptomatology consisted of dry cough in case A and in case B comprised fever, myalgia, malaise and sinus congestion (Fig. 1). Clinical examination findings were unremarkable. Initial investigations revealed only mild lymphopenia and elevation of CRP, with mild neutrophilia in case B. Periodic fever of $38-38.5^{\circ} \mathrm{C}$ was observed in case B until d3 of admission. Repeat blood tests in

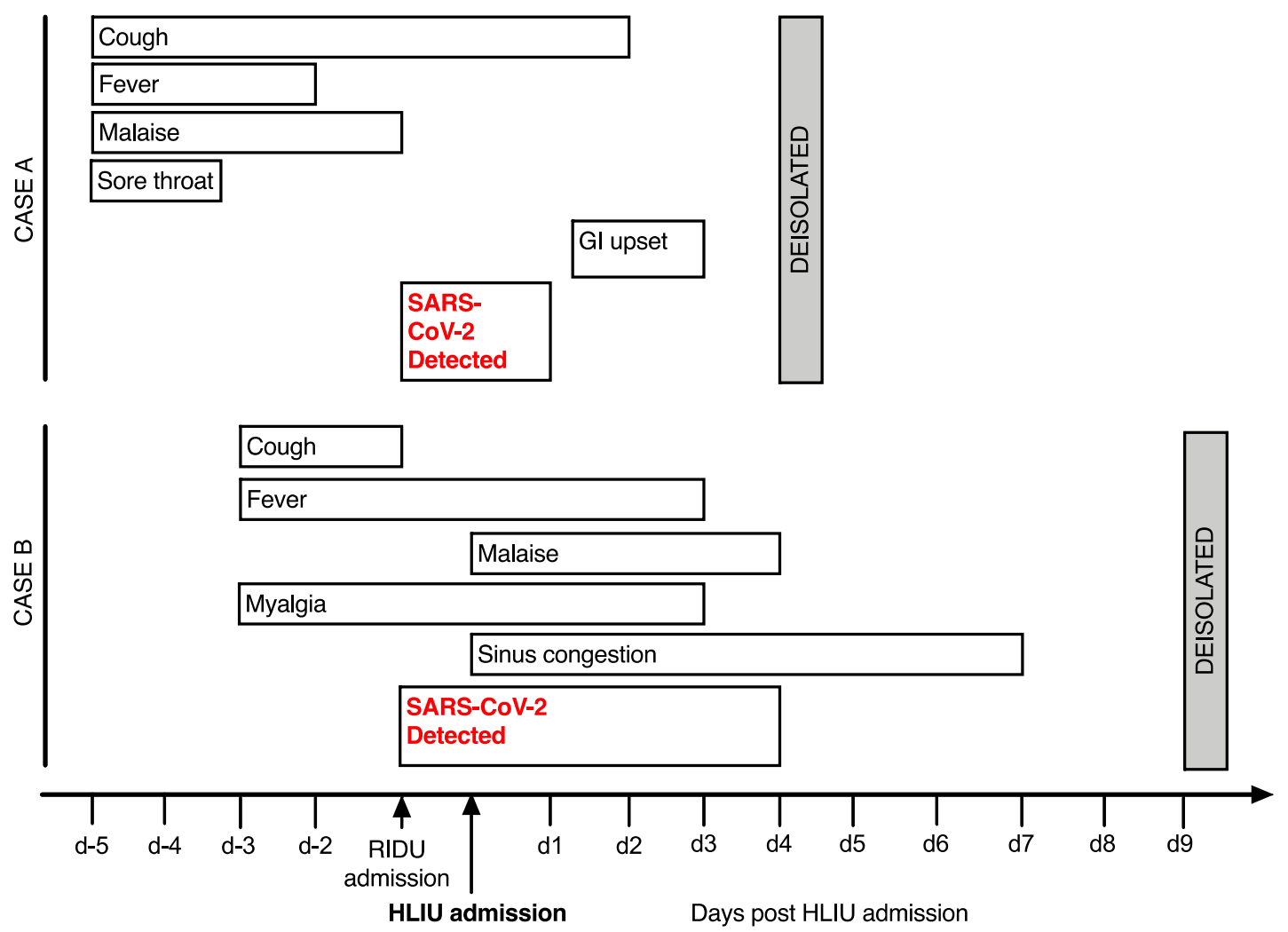

Fig. 1. Timeline of symptoms and SARS-CoV-2 PCR positivity on nasopharyngeal swabs. 
this individual on $\mathrm{d} 3$ demonstrated mild acute kidney injury (AKI, serum creatinine $144 \mu \mathrm{mol} / \mathrm{L}$ ). The AKI was thought most likely due to dehydration, and resolved within $24 \mathrm{~h}$ with administration of intravenous infusion of crystalloid at $125 \mathrm{ml} / \mathrm{h}$. CXR was normal. Empirical oral antibiotic therapy (co-amoxyclav 500/125 mg p.o. t.d.s.) was administered on $\mathrm{d} 3$, to cover the possibility of secondary bacterial infection, but was subsequently discontinued. Symptoms resolved in case A by $\mathrm{d} 3$ and in case B by $\mathrm{d} 4$ of admission. PCR testing of SARS-CoV-2 from nose and throat swabs taken daily was negative from $\mathrm{d} 2$ onwards in case $\mathrm{A}$ and from d5 in case B (throat swabs from this individual were negative throughout). There was no clinical indication for the use of experimental antiviral therapies. Patients were deisolated according to current PHE guidance, based on complete resolution of symptoms and two sequential negative respiratory PCR tests at least $24 \mathrm{~h}$ apart. Rooms were decontaminated with $0.1 \%$ hypochlorite followed by UV light treatment. The contact of these individuals remained asymptomatic throughout the 14 days incubation period but was isolated as a precaution and to be close to family

These first cases of SARS-CoV-2 are informative for clinicians caring for suspected and confirmed cases in the UK and elsewhere. Reassuringly, illness in both individuals was relatively mild and short-lived, with no evidence of parenchymal lung disease (reflected by normal oxygenation and the absence of radiological infiltrates) or of the late-stage deterioration that has been reported in case series ${ }^{9}$, possibly due to the absence of comorbidities. Experimental antiviral therapeutic options for severe disease were not considered necessary given the mild clinical nature of the illness. Clinical illness correlated with the presence of viral RNA in upper airway samples (Fig. 1), with no evidence of prolonged asymptomatic shedding, although discordance between nose and throat samples in case B highlights the need to sample both areas. It was reasonably assumed that the source of infection in case $B$ was close contact with symptomatic case A, given that the time from travel to China to onset of symptoms in case $B$ was 22 days, although this cannot be proven. Based on this assumption, the period from exposure to disease onset appeared short, at approximately $48 \mathrm{~h}$, consistent with recent reports of the incubation period of SARS-CoV-2 ${ }^{6}$. Co-occurrence of respiratory viral infection, as we observed in case $B$ with rhinovirus, has been described in the context of SARS-CoV-2 (https://www.medrxiv.org/ content/10.1101/2020.02.12.20022327v1) as it has with many other respiratory viruses spread by similar routes, and may have contributed to the increased symptomatology in case B. Interestingly the partner of case A, who was a close household contact, remained asymptomatic throughout and had negative tests for SARSCoV-2 shedding. It will be of interest to investigate the serological responses in this individual to ascertain evidence of subclinical infection.

Isolation, minimisation of contacts and use of appropriate PPE is a cornerstone of management of high consequence respiratory viral infection. In the cases reported here, PHE recommendations for PPE were followed ${ }^{10}$ and there were no breeches in PPE or nosocomial transmission. This should provide reassurance to healthcare workers managing patients with suspected Covid-19 in the UK that current PPE is both feasible and effective.

\section{Declaration of Competing Interest}

The authors declare that they have no known competing financial interests or personal relationships that could have appeared to influence the work reported in this paper.

\section{Acknowledgements}

We are grateful to the patients for providing their written informed consent to publish this report. Our thanks go to nursing, laboratory and medical colleagues in Hull University Teaching Hospitals NHS Trust and The Newcastle Upon Tyne Hospitals NHS Foundation Trust who contributed directly and indirectly to patient care, and to many colleagues in Public Health England and across the HCID network who contributed their time and expertise to the management of these cases. CJAD is supported by a Clinical Research Career Development Fellowship from the Wellcome Trust (211153/Z/18/Z).

\section{References}

1. Tang J.W., Tambyah P.A., Hui D.S.C.. Emergence of a novel coronavirus causing respiratory illness from Wuhan, China. J Infect 2020 published online Jan 27. doi:10.1016/j.jinf.2020.01.014

2. $\mathrm{Q}$ L.i.The $\mathrm{nCoV}$ Outbreak Joint Field Epidemiology Investigation Team An outbreak of NCIP (SARS-CoV-2) infection in China - Wuhan, Hubei province, 2019-2020. CCDC Weekly 2020;2:80-1

3. Zhu N., Zhang D., Wang W., et al. A novel coronavirus from patients with pneumonia in China, 2019. N Engl J Med 2020 published online Jan 24. doi:10.1056/ NEJMoa2001017

4. Chan J.F.-W., Yuan S., Kok K.-.H., et al. A familial cluster of pneumonia associated with the 2019 novel coronavirus indicating person-to-person transmission: a study of a family cluster. Lancet 2020;395:514-23.

5. Li Q., Guan X., Wu P., et al. Early transmission dynamics in wuhan, china, of novel coronavirus-infected pneumonia. N Engl J Med 2020 published online Jan 29. doi:10.1056/NEJMoa2001316.

6. Phan L.T., Nguyen T.V., Luong Q.C., et al. Importation and human-to-human transmission of a novel coronavirus in Vietnam. N Engl J Med 2020 published online Jan 28, doi:10.1056/NEJMc2001272.

7. Rothe C., Schunk M., Sothmann P., et al. Transmission of SARS-CoV-2 infection from an asymptomatic contact in Germany. N Engl J Med 2020 published online Jan 30. doi:10.1056/NEJMc2001468.

8. Holshue M.L., DeBolt C., Lindquist S., et al. First case of 2019 novel coronavirus in the United States. N Engl J Med 2020 published online Jan 31. doi:10.1056/ NEJMoa201191.

9. Huang C., Wang $Y$, Li $X$, et al. Clinical features of patients infected with 2019 novel coronavirus in Wuhan, China. The Lancet 2020:395: 497-506.

10. Novel coronavirus (SARS-CoV-2) infection prevention and control guidance. Public Health England. www.gov.uk/government/publications/wuhan-novelcoronavirus-infection- prevention-and-control/wuhan-novel-coronavirus-wncov-infection-prevention-and-control-guidance Accessed 3rd February 2020.

Patrick J. Lillie, Anda Samson

Department of Infection, Hull University Teaching Hospitals NHS Trust, Hull, United Kingdom

Ang Li

Department of Infection and Tropical Medicine, The Newcastle Upon Tyne Hospitals NHS Foundation Trust, Newcastle upon Tyne, United Kingdom

Kate Adams

Department of Infection, Hull University Teaching Hospitals NHS Trust, Hull, United Kingdom

Richard Capstick Department of Infection and Tropical Medicine, The Newcastle Upon Tyne Hospitals NHS Foundation Trust, Newcastle upon Tyne, United

Kingdom

Gavin D. Barlow Department of Infection, Hull University Teaching Hospitals NHS Trust, Hull, United Kingdom

Hull York Medical School, University of York, United Kingdom

Nicholas Easom

Department of Infection, Hull University Teaching Hospitals NHS Trust, Hull, United Kingdom 
Eve Hamilton

Department of Infection and Tropical Medicine, The Newcastle Upon Tyne Hospitals NHS Foundation Trust, Newcastle upon Tyne, United

Kingdom

Peter J. Moss

Department of Infection, Hull University Teaching Hospitals NHS Trust, Hull, United Kingdom

Adam Evans

Department of Infection and Tropical Medicine, The Newcastle Upon Tyne Hospitals NHS Foundation Trust, Newcastle upon Tyne, United

Kingdom

Monica Ivan

Department of Infection, Hull University Teaching Hospitals NHS Trust, Hull, United Kingdom

PHE Incident Team PHE Incident Team, Public Health England, National Infection Service, London NW9 5EQ United Kingdom

Yusri Taha Department of Infection and Tropical Medicine, The Newcastle Upon Tyne Hospitals NHS Foundation Trust, Newcastle upon Tyne, United Kingdom

Christopher J.A. Duncan* Department of Infection and Tropical Medicine, The Newcastle Upon Tyne Hospitals NHS Foundation Trust, Newcastle upon Tyne, United Kingdom

Translational and Clinical Research Institute, Immunity and Inflammation Theme, Newcastle University, United Kingdom

Matthias L. Schmid ${ }^{* *}$ Department of Infection and Tropical Medicine, The Newcastle Upon Tyne Hospitals NHS Foundation Trust, Newcastle upon Tyne, United Kingdom

the Airborne HCID Network ${ }^{1}$ Airborne HCID Network, United Kingdom

*Corresponding author at: Department of Infection and Tropical Medicine, The Newcastle Upon Tyne Hospitals NHS Foundation Trust, Newcastle upon Tyne, United Kingdom. **Corresponding author.

E-mail addresses: christopher.duncan@ncl.ac.uk (C.J.A. Duncan), Matthias.schmid1@nhs.net (M.L. Schmid)

1 Nicholas M Price (Network Lead); Department of Infection, Guy's and St Thomas' NHS Trust. Michael Jacobs; Department of Infection, Royal Free Hospital NHS Trust.

Michael BJ Beadsworth; Tropical and Infectious Disease Unit, Royal Liverpool University Hospital. Matthias L Schmid; Department of Infection \& Tropical Medicine, Newcastle upon Tyne Hospitals NHS FT.

Anne Tunbridge; Department of Infectious Diseases, Sheffield Teaching Hospitals NHS FT. Accepted 25 February 2020 Available online 15 January 2020

https://doi.org/10.1016/j.jinf.2020.02.020

0163-4453/@ 2020 Published by Elsevier Ltd on behalf of The British Infection Association.
Simulating and forecasting the cumulative confirmed cases of SARS-CoV-2 in China by Boltzmann function-based regression analyses

Dear editor,

As reported in this Journal ${ }^{1}$ and elsewhere, ${ }^{2}$ an outbreak of atypical pneumonia caused by the zoonotic 2019 novel coronavirus (SARS-CoV-2) is on-going in China and has spread to the world. As of Feb 16, 2020 (24:00, GMT+8), there have been 70,548 confirmed patients and more than 1700 deaths from SARS-CoV-2 infection in China, and 58,182 confirmed patients and 1696 deaths in the most affected province, Hubei Province. Much research progress has been made in dissecting the evolution and origin of SARS-CoV2 and characterizing its clinical features. ${ }^{3-7}$

While the outbreak is on-going, people raise grave concerns about the future trajectory of the outbreak, especially given that the working and schooling time has been already dramatically postponed after the Chinese Lunar New Year holiday was over (scheduled on Jan 31). In particular, a precise estimation of the potential total number of infected cases and/or confirmed cases is highly demanding. Earlier studies based on susceptible-exposed-infectious-recovered metapopulation and susceptible-infected-recovered-dead models revealed the number of potentially infected cases and the basic reproductive number of SARS-CoV-2.3,8,9 These traditional epidemiological models apparently require much detailed data for analysis. ${ }^{3,8}$

Here we explored a simple data-driven, Boltzmann functionbased approach for estimation only based on the daily cumulative number of confirmed cases of SARS-CoV-2 (Note: the rational for Boltzmann function-based regression analysis is presented in supporting information (SI) file). We decided to collect data (initially from Jan 21 to Feb 10, 2020) in several typical regions of China, including the center of the outbreak (i.e. Wuhan City and Hubei Province), other four most affected provinces (i.e., Guangdong, Zhejiang, Henan, Hunan) and top-4 major cities in China (i.e., Beijing, Shanghai, Guangzhou, Shenzhen). During data analysis on Feb 13, 2020, the number of new confirmed cases on Feb 12 in Hubei Province and Wuhan City suddenly increased by 14,840 and 13,436 , respectively, of which 13,332 and 12,364 are those confirmed by clinical features (note: all the number of confirmed cases released by Feb 12 were counted according to the result of viral nucleic acid detection rather than by referring to clinical features). We thus arbitrarily distributed these suddenly added cases to the reported cumulative number of confirmed cases from Jan 21 to Feb 14 for Hubei Province by a fixed factor (refer to Table S1), assuming that they were linearly accumulative in those days. It is the same forth with the data for Wuhan City.

Regression analyses indicate that all sets of data were well fitted with the Boltzmann function (all $R^{2}$ values being close to 0.999; Figs. 1A, B, S1, and Table 1). The potential total number of confirmed cases for mainland China, Hubei Province, Wuhan City, and other provinces were estimated as $72,800 \pm 600,59,300 \pm 600$, $42,100 \pm 700$ and $12,800 \pm 100$; respectively; those for provinces Guangdong, Zhejiang, Henan and Hunan were $1300 \pm 10,1170 \pm 10$, $1260 \pm 10,1050 \pm 10,1020 \pm 10$ and $940 \pm 10$, respectively (Table 1); those for Beijing, Shanghai, Guangzhou and Shenzhen were $394 \pm 4$, $328 \pm 3,337 \pm 3$ and $397 \pm 4$, respectively. In addition, we estimated the key date, on which the number of daily new confirmed cases is lower than $0.1 \%$ of the potential total number as defined by us subjectively (refer to Table 1 ).

The above analyses were performed assuming that the released data on the confirmed cases are precise. However, there is a 

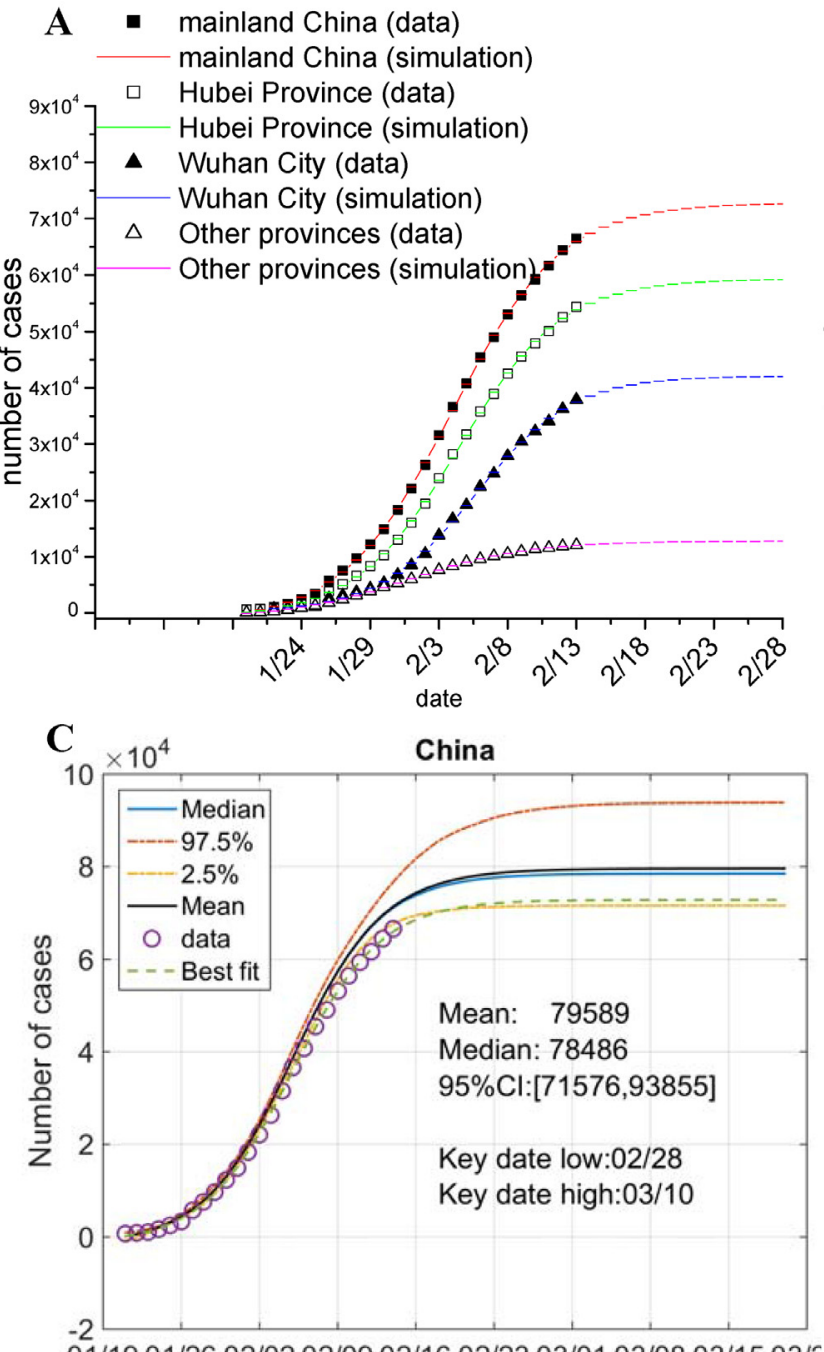

01/19 01/26 02/02 02/09 02/16 02/23 03/01 03/08 03/15 03/2

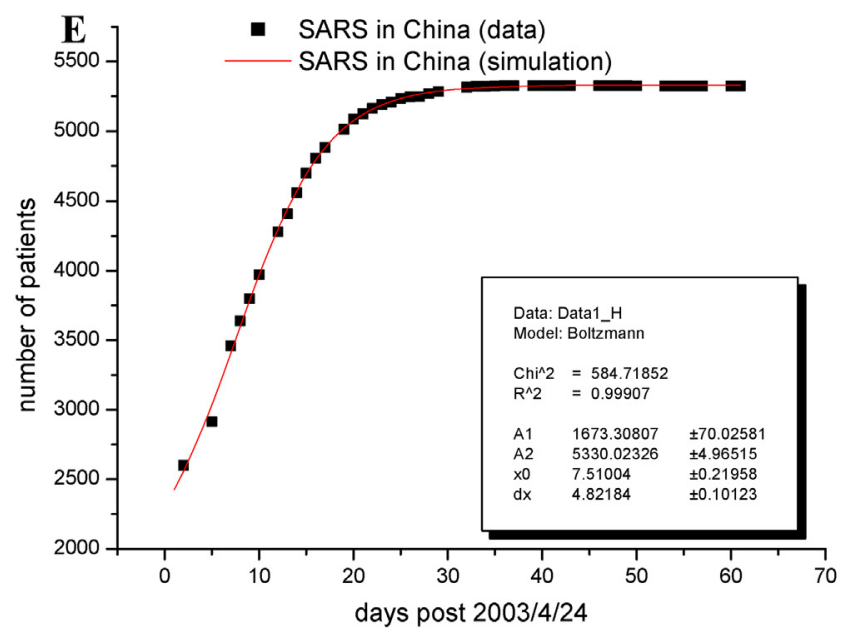

B

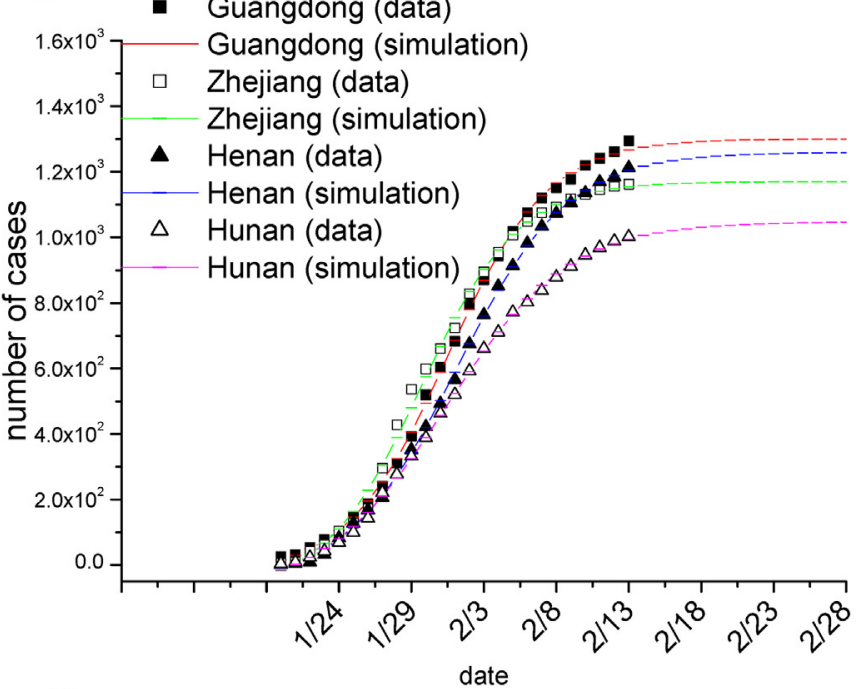

D

date

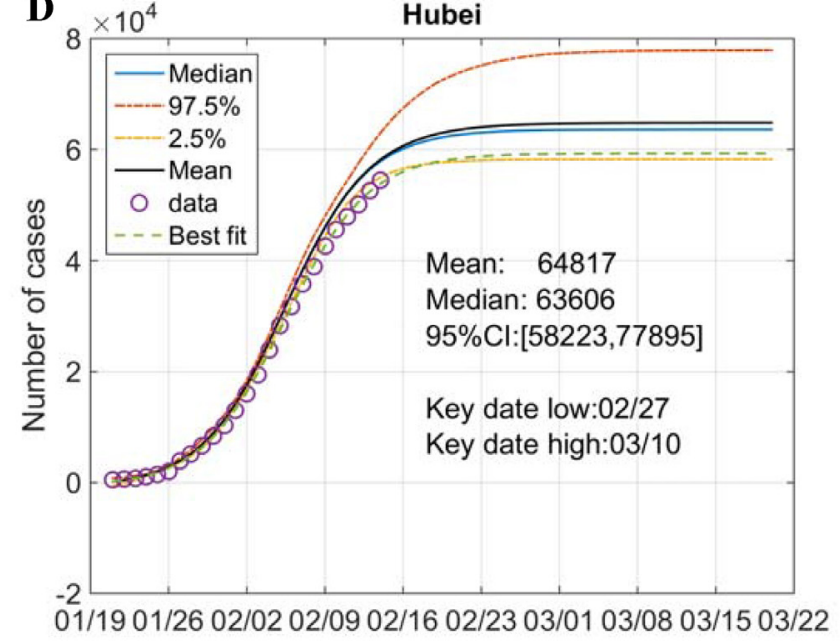

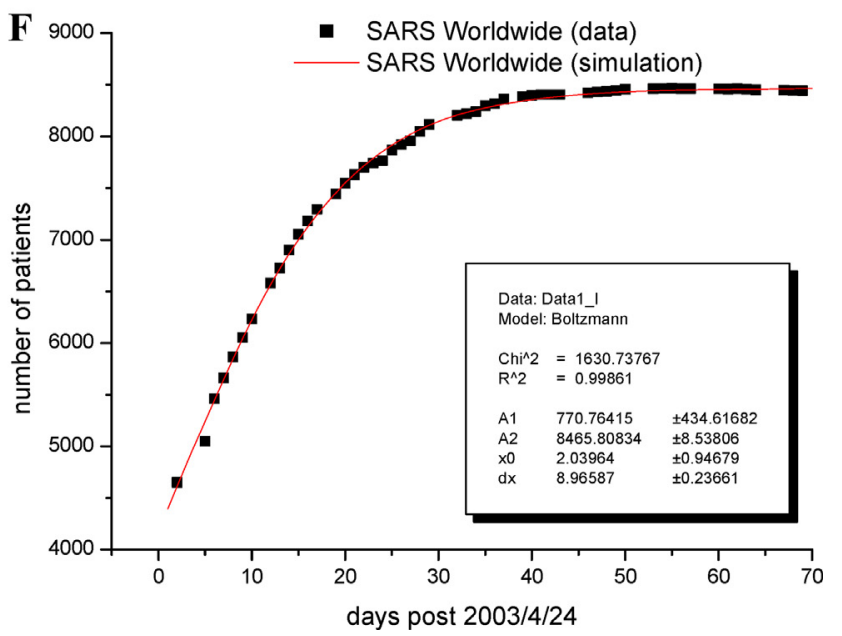

Fig. 1. Fitting the cumulative number of confirmed cases from different geographic regions of China to the Boltzmann function.

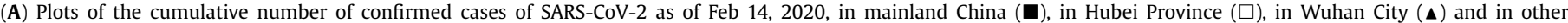

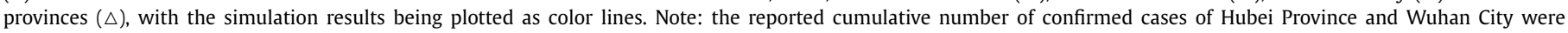

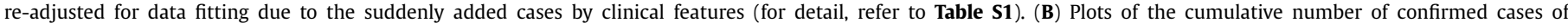

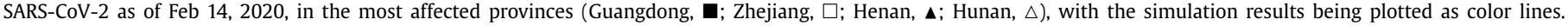

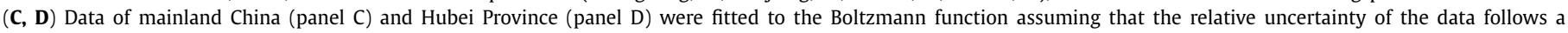

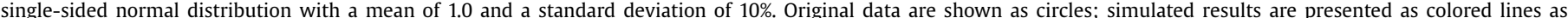

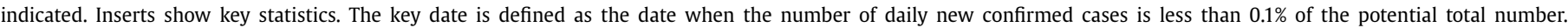

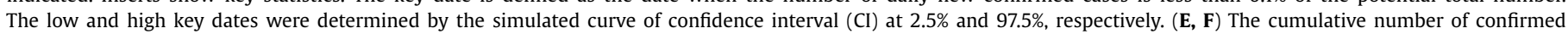

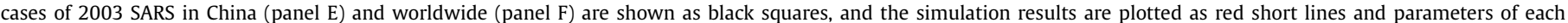
established function are shown in inserts. (For interpretation of the references to colour in this figure legend, the reader is referred to the web version of this article.) 
Table 1

Regression analysis results of confirmed cases of SARS-CoV-2 in China.

\begin{tabular}{|c|c|c|c|c|c|}
\hline Regions & \multicolumn{3}{|l|}{ without uncertainty } & \multicolumn{2}{|l|}{ with uncertainty ${ }^{\mathrm{a}}$} \\
\hline China & $72,800 \pm 600$ & $2 / 28$ & 0.999 & $79,589(71,576,93,855)$ & $(2 / 28,3 / 10)$ \\
\hline Wuhan City & $42,100 \pm 700$ & $2 / 27$ & 0.999 & $46,562(40,812,57,678)$ & $(2 / 28,3 / 10)$ \\
\hline Other provinces & $12,800 \pm 100$ & $2 / 27$ & 0.999 & $13,956(12,748,16,092)$ & $(2 / 27,3 / 13)$ \\
\hline Guangdong Province & $1300 \pm 10$ & $2 / 22$ & 0.999 & $1415(1324,1550)$ & $(2 / 22,3 / 01)$ \\
\hline Henan Province & $1260 \pm 10$ & $2 / 24$ & 0.999 & $1372(1271,1559)$ & $(2 / 26,3 / 09)$ \\
\hline Hunan Province & $1050 \pm 10$ & $2 / 26$ & 0.999 & $1140(1050,1279)$ & $(2 / 28,3 / 11)$ \\
\hline Beijing City & $394 \pm 4$ & $2 / 25$ & 0.999 & $429(395,486)$ & $(2 / 25,3 / 11)$ \\
\hline Shanghai City & $328 \pm 3$ & $2 / 22$ & 0.999 & $356(334,388)$ & $(2 / 22,3 / 01)$ \\
\hline Guangzhou City & $337 \pm 3$ & $2 / 20$ & 0.998 & $365(346,393)$ & $(2 / 20,2 / 28)$ \\
\hline Shenzhen City & $397 \pm 4$ & $2 / 18$ & 0.998 & $430(407,461)$ & $(2 / 17,2 / 25)$ \\
\hline
\end{tabular}

a The reported cumulative number of confirmed cases may have uncertainty. Assuming the relative uncertainty follows a single-sided normal distribution with a mean of 1.0 and a standard deviation of $10 \%$, the potential total number and key dates were estimated at $95 \%$ CI. For detail, refer to the Methods section and Figs. 1C, D, S2 and S3.

b Key date is determined when the number of daily new confirmed cases is less than $0.1 \%$ of the potential total number.

tendency to miss-report some positive cases such that the reported numbers represent a lower limit. One typical example indicating this uncertainty is the sudden increase of more than 14000 new confirmed cases in Hubei Province on Feb 12 after clinical features were officially accepted as a standard for infection confirmation. Another uncertainty might result from insufficient kits for viral nucleic acid detection at the early stage of the outbreak. We thus examined the effects of such uncertainty using a Monte Carlo method (for detail, refer to the Methods section in SI file). For simplicity, we assumed that the relative uncertainty of the reported data follows a single-sided normal distribution with a mean of 1.0 and a standard deviation of $10 \%$.

Under the above conditions, the potential total numbers of confirmed cases of SARS-CoV-2 for different regions were estimated (Figs. 1C, D, S2 and S3) and summarized in Table 1. The potential total numbers for China, Hubei Province, Wuhan City and other provinces were 79,589 (95\% CI 71,576, 93,855), 64,817 (58,223, $77,895), 46,562(40,812,57,678)$ and $13,956(12,748,16,092)$, respectively, indicating that overall the outbreak may not be so bad as previously estimated. ${ }^{9}$ Such uncertainty analysis also allowed us to estimate the key dates at $95 \% \mathrm{CI}$. As summarized in Table 1, the key dates for mainland China, Hubei Province, Wuhan City, and other provinces would fall in $(2 / 28,3 / 10),(2 / 27,3 / 10),(2 / 28,3 / 10)$ and $(2 / 27,3 / 13)$, respectively.

Finally, the ongoing SARS-CoV-2 outbreak has undoubtedly thus collected the data from the WHO officiate website for analysis, and found that the cumulative numbers of confirmed cases of 2003 SARS-CoV both in China and worldwide were fitted well with the Boltzmann function, with $R^{2}$ being 0.999 and 0.998 , respectively (Figs. 1E and F).

In summary, we found that all data sets, including both the on-going outbreak of SARS-CoV-2 in China and the 2003 SARS-CoV epidemic in China and worldwide, were well fitted to the Boltzmann function (Fig. 1 and $\mathbf{S 1}$ ). These results strongly suggest that the Boltzmann function is suitable for analyzing the epidemics of coronaviruses like SARS-CoV and SARS-CoV-2. One advantage of this model is that it only needs the cumulative number of confirmed cases, somehow as simple as the recently proposed model. ${ }^{10}$ In addition, the estimated potential total numbers of confirmed cases and key dates may provide valuable guidance for Chinese central and local governments to deal with this emerging threat at current critical stage. caused us the memories of the SARS-CoV outbreak in 2003. We

\section{Acknowledgments}

We thank graduate students (Boyan Lv, Zhongyan Li, Zhongyu Chen, Yu Cheng, Mengmeng Bian, Shuang Zhang, Zuqin Zhang, and Wei Yao; all from Prof. Xinmiao Fu's research group at Fujian Normal University) for data collection. This work is support by the National Natural Science Foundation of China (No. 31972918 and 31770830 to $\mathrm{XF}$ ).

\section{Supplementary materials}

Supplementary material associated with this article can be found, in the online version, at doi:10.1016/j.jinf.2020.02.019.

\section{References}

1. Tang J.W., Tambyah P.A., Hui D.S.C.. Emergence of a novel coronavirus causing respiratory illness from Wuhan, China. J Infect 2020. doi:10.1016/j.jinf.2020.01. 014

2. Wang C., et al. A novel coronavirus outbreak of global health concern. Lancet 2020. doi:10.1016/S0140-6736(20)30185-9.

3. Yang Y., et al., Epidemiological and clinical features of the 2019 novel coronavirus outbreak in China. doi:10.1101/2020.02.10.20021675, 2020.

4. Wu F., et al. A new coronavirus associated with human respiratory disease in China. Nature 2020 (2020). doi:10.1038/s41586-020-2008-3.

5. Zhou P., et al. A pneumonia outbreak associated with a new coronavirus of probable bat origin. Nature 2020 (2020). doi:10.1038/s41586-020-2012-7.

6. Lu R., et al. Genomic characterisation and epidemiology of 2019 novel coronavirus: implications for virus origins and receptor binding. Lancet 2020. doi:10 1016/s0140-6736(20)30251-8.

7. Guan W.J., et al., Clinical characteristics of 2019 novel coronavirus infection in China. doi:10.1101/2020.02.06.20020974, 2020.

8. Wu J.T., Leung K., Leung G.M.. Nowcasting and forecasting the potential domestic and international spread of the 2019-nCoV outbreak originating in wuhan, china: a modelling study. Lancet 2020. doi:10.1016/S0140-6736(20)30260-9.

9. Anastassopoulou, C., et al., Data-based analysis, modelling and forecasting of the novel coronavirus (2019-NCOV) outbreak. doi:10.1101/2020.02.11.20022186, 2020.

10. Huang N.E., Qiao F.. A data driven time-dependent transmission rate for tracking an epidemic: a case study of 2019-nCoV. Sci Bull 2020. doi:10.1016/j.scib.2020. 02.005 .

Xinmiao $\mathrm{Fu}^{*}$

Provincial University Key Laboratory of Cellular Stress Response and Metabolic Regulation, College of Life Sciences, Fujian Normal University (Qishan campus), Room 214, Ligong Building, Fuzhou, Fujian 350117, China

Qi Ying

Department of Civil and Environmental Engineering, Texas AEM University, College Station, TX 77843, USA

Tieyong Zeng

None.
Department of mathematics, The Chinese University of Hong Kong, Shatin, NT, Hong Kong 99999, China 
Tao Long

Nanjing Institute of Environmental Sciences, Ministry of Ecology and Environment, China

Yan Wang

Provincial University Key Laboratory of Cellular Stress Response and Metabolic Regulation, College of Life Sciences, Fujian Normal University (Qishan campus), Room 214, Ligong Building, Fuzhou, Fujian 350117, China

*Corresponding author. E-mail address: xmfu@fjnu.edu.cn (X. Fu)

Accepted 21 February 2020 Available online 15 January 2020

https://doi.org/10.1016/j.jinf.2020.02.019

(c) 2020 The British Infection Association. Published by Elsevier Ltd. All rights reserved.

\section{Chinese medical personnel against the 2019-nCoV}

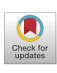

We appreciate the work Tang et al. have report emergence of a novel coronavirus in China. ${ }^{1}$ The $2019-n C o V$ broke out in Wuhan,
China at the end of 2019, and has attracted worldwide attention. ${ }^{2-4}$ Although the Chinese government has taken active measures to control this epidemic, the virus is very infectious. According to the real-time data of the National Health Commission of the People's Republic of China up until February 5, 2020, within a short period of half a month, the number of confirmed cases and the number of deaths were 24,443 and 493 , respectively. The epidemic is progressing rapidly. 2019-nCoV poses new public health challenges in China. ${ }^{5}$ In Wuhan, China, the number of local medical staff is insufficient for the demand resulting from the explosive increase in the number of infected patients. Therefore, many medical personnel are needed to devote themselves to the front line of combating the virus.

Medical personnel throughout the country are led under the unified leadership of the Chinese government. Although the epidemic in Wuhan is serious, a large number of medical staff rushed to Wuhan to supplement the shortage of manpower in Wuhan hospitals. This is a battle without smoke, the heroes of which are our medical staff. According to the National Health Commission of the People's Republic of China, as of January 30, 2020, Hubei Province opened 11,000 isolated patient beds, and about 170,000 healthcare professionals from all kinds of medical institutions are working on the front lines and providing care for patients with fevers, and for suspected or confirmed patients. In this time of emergency, under the unified deployment of the Chinese government, there are 52 medical teams including 6097 medical personnel from the National

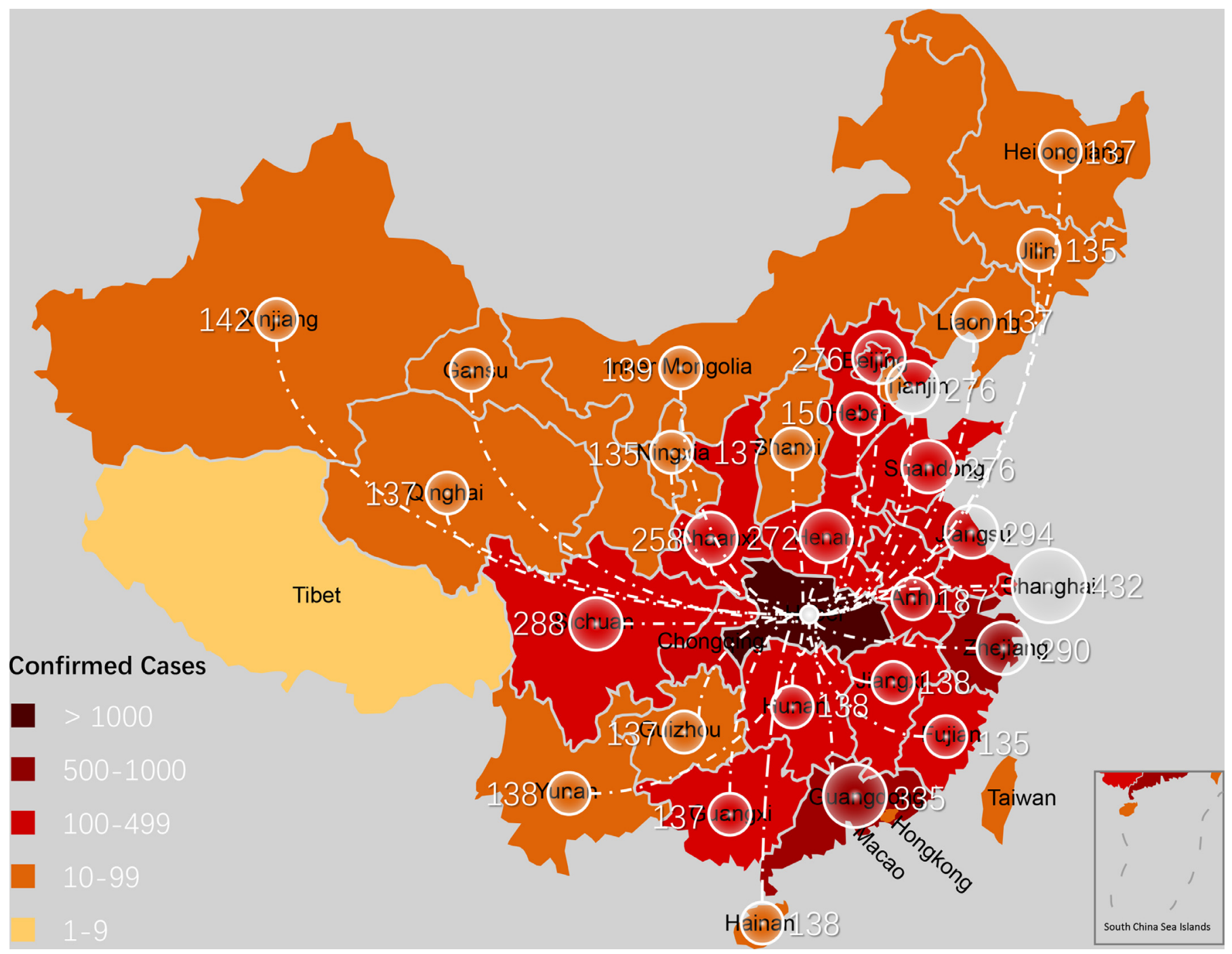

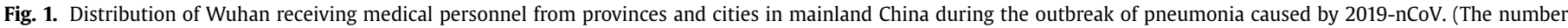

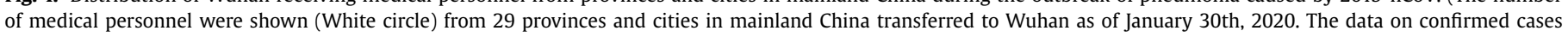
(Colored block) were obtained from the websites of Chinese National Health Commissions). 
Health Commission, the State Administration of Traditional Chinese Medicine, the Academy of Chinese Medical Sciences, 29 provinces and cities, as well as the army (Fig. 1).

Huoshenshan Hospital is a specialized hospital established in the Wuhan Staff Sanatorium.

Patients with confirmed coronavirus pneumonia have been admitted to our hospital. It has a total of 1000 beds, and includes an intensive care unit, an ordinary care unit, a laboratory, and radiology and other auxiliary departments. According to the National Health Commission of the People's Republic of China, the related design scheme of the institute was completed on January 24, 2020. Construction of the hospital began on January 29th, and the hospital was completed and put into use on February 2nd. The Chinese People's Liberation Army has transferred 1400 medical personnel to undertake the task of helping people infected with the virus.

We firmly believe that Chinese medical personnel and people throughout the country can work together to win this defensive battle with one heart and one mind.

\section{References}

1. Tang J.W., Tambyah P.A., Hui D.S.C.. Emergence of a novel coronavirus causing respiratory illness from Wuhan, China. J Infect 2020. pii:S0163-4453(20)300384. doi: 10.1016/j.jinf.2020.01.014.

2. Li Q., Guan X., Wu P., Wang X., Zhou L., Tong Y., et al. Early transmission dynamics in Wuhan, China, of novel coronavirus-infected pneumonia. $N$ Engl J Med 2020. doi:10.1056/NEJMoa2001316.

3. Huang C., Wang Y., Li X., Ren L., Zhao J., Hu Y., et al. Clinical features of patients infected with 2019 novel coronavirus in Wuhan,China. Lancet 2020. pii:S01406736(20)30183-5. doi: 10.1016/S0140-6736(20)30183-5.

4. Phelan A.L., Katz R., Gostin L.O.. The novel coronavirus originating in Wuhan. China: Challenges for Global Health Governance. JAMA. 2020. doi:10.1001/jama. 2020.1097.

5. Wang R., Zhang X., Irwin D.M., Shen Y.. Emergence of SARS-like coronavirus poses new challenge in China. J Infect 2020. pii:S0163-4453(20)30057-8. doi: 10.1016/j.jinf.2020.01.017.
Zhan-hui Feng ${ }^{1}$

Department of Neurology, Affiliated Hospital of Guizhou Medical University, Guiyang, China

Yong-ran Cheng ${ }^{1}$

Zhejiang Academy of Medical Sciences, Hangzhou 310012, China Hangzhou Medical College, Hangzhou 311300, China

Juan Chen

Affiliated Hospital of Hangzhou Normal University, Hangzhou 310015, China

Lan Ye

Basic Medical College, Guizhou Medical University, Guizhou 550004,

China

Meng-Yun Zhou

Hangzhou Medical College, Hangzhou 311300, China

Ming-Wei Wang*

Affiliated Hospital of Hangzhou Normal University, Hangzhou

310015, China

*Corresponding author.

E-mail address: wmw990556@163.com (M.-W. Wang)

${ }^{1}$ These authors are contributed equally to this work. Accepted 7 February 2020 Available online 15 January 2020

https://doi.org/10.1016/j.jinf.2020.02.011

(c) 2020 The British Infection Association. Published by Elsevier Ltd. All rights reserved. 